\title{
Understanding of the major reactions in solution synthesis of functional nanomaterials
}

\author{
Yuen $\mathrm{Wu}^{1,2}$, Dingsheng Wang ${ }^{1}$ and Yadong $\mathrm{Li}^{1 *}$
}

\begin{abstract}
This review covers the major reactions involved in the solution synthesis of nanomaterials. It was designed to classify the traditional strategies such as precipitation, reduction, seed growth, etching, and so on into two basic processes which are termed as bottom-up and top-down routines. The discussion is focused on the basic mechanism and principles during the nucleation and growth of nanocrystals, especially in the solution system. This review also presents a prediction for how to utilize these intrinsic processes to artificially construct the desired specific and functional nanostructures. We try to describe the most directive and effective way to control the structures of nanocrystals for researchers who can master the major reaction mechanism and grasp the basic technologies in synthetic nanoscience.
\end{abstract}

Keywords: nanomaterials, solution synthesis, bottom-up, topdown, nanocrystals

\section{INTRODUCTION}

Since unique chemical and physical properties emerge when the size scale is down to nanoscale, inorganic nanomaterials such as metal, oxide and semiconductor, have gradually drawn increased interests in a variety of research fields such as electronics, catalysis and optics. The properties of nanomaterials are not only related to their intrinsic matter themselves but also their existence form such as sizes, compositions, crystal phases, surface facets, and so on. The synthesis of nanocrystals (NCs) is a prerequisite topic for their further performance tests and applications. During recent decades, abundant efforts have been paid to develop the general strategy to synthesize and control the nanostructure. For instance in physical methods, chemical vapour deposition (CVD) has been widely utilized to prepare two-dimensional (2D) materials such as graphene-based composites, and metal dichalcogenides. Owning to the merits such as high yield, versatile manipulation, systematical control, low energy consumption and air pollution, well-defined structure and so on, solution-based methods have gained great successes in preparation of nanomaterials among all the developed synthetic methods.

Although manipulating every single atom in solution is difficult at present, researchers have contributed great efforts in the controllable synthesis of various nanomaterials with homogeneous size, uniform morphology, and well-defined structure. In 2007, our group reviewed the synthesis of monodispersed NCs by carefully designing the interface between air, solid and solution [1]. In 2009, Xia's group [2] summarized the basic chemistry and physical rules in controlling the shape of metal NCs. Further, the progresses in branched [3], concave [4], and high-index facet [5-7] metallic structures were also summarized and reviewed by various research groups. Chen et al. [8] discussed the roles of small adsorbates in tailoring the surface facets and surface atomic configuration of Pd and Pt based NCs. Liu et al. [9] concluded how to utilize the template to regulate the structure of nanomaterials. Recently, Huang's group [10] demonstrated and reviewed the biomimetic molecules that can also be adopted as the capping agent to modulate the surface structures of NCs. Solvothermal/hydrothermal methods are also effective tools, which usually occur in a sealed heated solution above ambient temperature and pressure, in synthesis of nanomaterials such as semiconducting nanostructures [11] and oxides [12]. Actually, the energy activating the reactions in solution can also be introduced from the exterior reaction environments such as the microwave and ultrasonic. Nadagouda et al. [13] provided an overview of microwave-assisted synthetic approach in

\footnotetext{
${ }^{1}$ Department of Chemistry and Collaborative Innovation Center for Nanomaterial Science and Engineering, Tsinghua University, Beijing 100084, China ${ }^{2}$ Department of Chemistry and Center of Advanced Nanocatalysis, University of Science and Technology of China, Hefei 230026, China

* Corresponding author (email: ydli@mail.tsinghua.edu.cn)
} 
synthesizing the Ag nanostructures, which can be further extended to the oxides and chalcogenides. Afterwards, Suslick's group summarized the synthesis of nanomaterials by sonochemical procedures [14].

This review is focused on the major reactions in the synthetic approaches during the solution-based synthesis of colloidal nanoparticles (NPs). In tradition, strategies for fabrication of functional nanostructures can be defined as either "bottom-up" or "top-down" processes. Starting with atoms, molecules, clusters or even NPs, the "bottom-up" approach enables the synthesis of multitudinous nanostructures by assembly from these "building blocks". On the contrary, "top-down" methods involving the carving of pre-formed architectures allow to alter the size/geometry-dependent electronic structures considerably, and assist the sophisticated design of specific properties. Understanding the inherent features of reactions occurring in the solvent allows for better controlling the size, composition, and structure of nanomaterials. We discuss representative reactions for both the bottom-up and top-down synthesis, namely precipitation, reduction, and decomposition/hydrolysis, for bottom-up strategy, replacement, chemical etching, cation exchange reactions, and exfoliation for top-down strategy. So far as the present research, the development of bottom-up process mainly focuses on the goal of versatile and generalized molecular-level control of nanomaterials under rational design and predictable structures (Scheme 1). Moreover, a brief overview combining the bottom-up and top-down strategies in the aim of fabricating more complex and elaborate nanostructures and sequential bottom-up strategy in the purpose for hierarchical structure are talked about.

In general, the solution-based synthesis mainly involves the nucleation and growth of NCs and the solubility changes of substances in hydrophilic or hydrophobic solution. There are several parameters that should be considered during the preparation of nanomaterials in solution. For intrinsic reaction system conditions, the concentration of precursors, $\mathrm{pH}$ value and viscosity of the solution, reaction temperature and time, pressure of the reaction system, capping agents, and so on play a vital role in controlling the reaction rate and equilibrium. Although the exterior reaction environment conditions such as air bubble [15], microwave, ultrasonic, and magnetic field [16-19] can also bring significant influence to the reaction system, we mainly select the interior factors as the emphasis to discuss the fundamental understanding and basic principles in solution synthesis of nanomaterials. Ultimately, we try to provide a clue that how to adopt the right reactions to fabricate the desired nanostructures and push for the development in solution synthesis of nanomaterials.

\section{BASIC CONCEPTS ON NUCLEATION AND GROWTH IN SOLUTION}

In solution synthesis of nanomaterials, the synthetic process can be generally divided into nucleation and growth process. The nucleation can be recognized as the precursors are firstly dissolved or dispersed in the solvent and subsequently produce the nuclei or clusters, which are

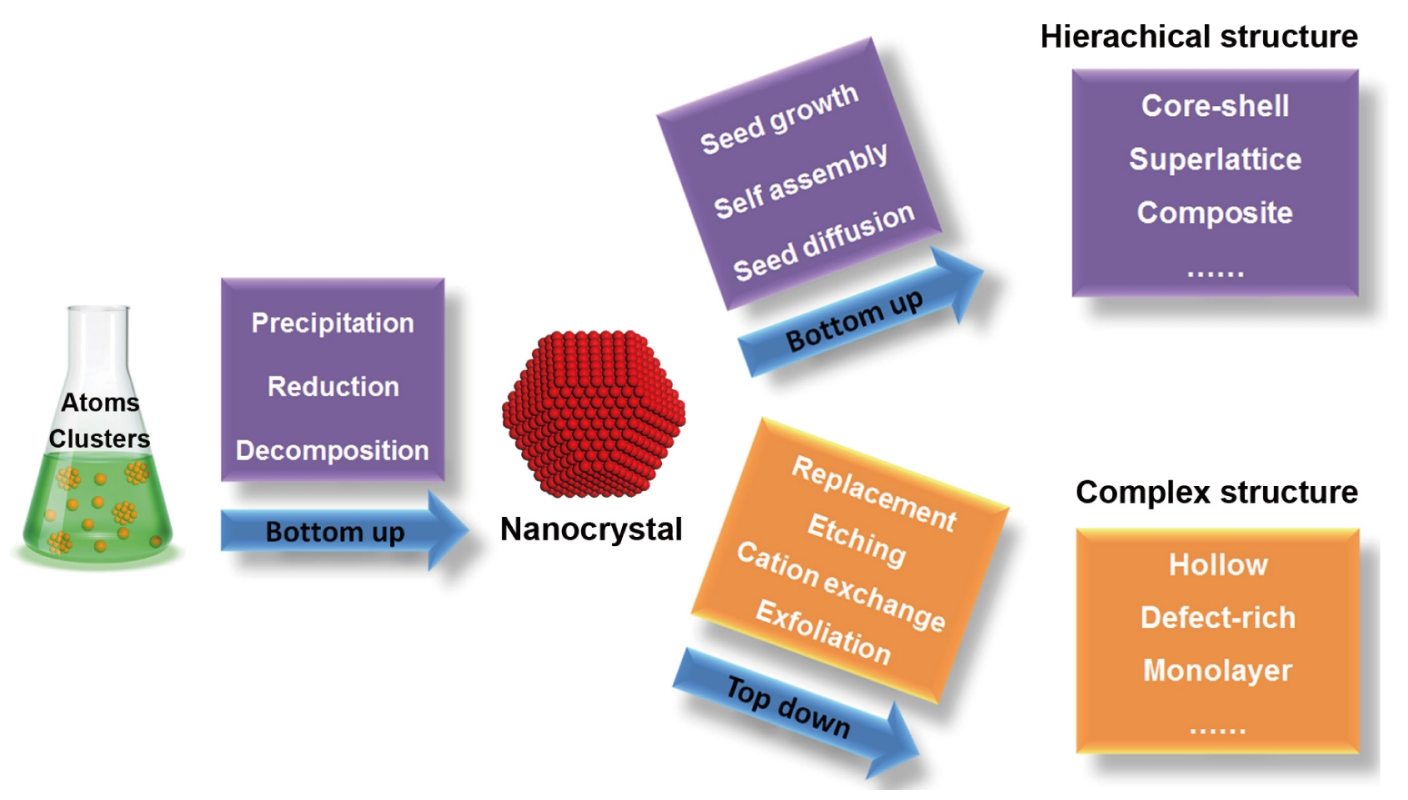

Scheme 1 Schematic representation of the bottom-up and top-down strategies in the synthesis of nanomaterials. 
new-phase materials generated from the solution. The nucleation process is the birth of NCs and plays a key role in the crystal structure, size distribution, morphology, and intrinsic geometric and electrical structures of the final nanomaterials. Understanding the mechanism and principles of nucleation may help us to choose the right starting materials and design the correct reaction systems.

\section{Nucleation process}

Because the nucleation is very fast and the involved subnano structures such as minuscule clusters consist of very few atoms, capturing and manipulating this process is very difficult. Nevertheless, substantial efforts have been paid to clarify this "holy grails" in nanoscience. Because of the analytical simplicity, nucleation theory gained the first breakthrough in the solution crystallization. In 1950, LaMer's group [20] proposed the initial nucleation mechanism for a typical synthesis of sulfur colloids in solution. There are two crucial equations involved in their theoretical model in aim of elucidating the nucleation process. Assuming the nuclei are with spherical shape, the free energy change $(\Delta G)$ of the nucleation process can be calculated as

$$
\Delta G=-\frac{4}{V} \pi r^{3} k_{\mathrm{B}} T \ln (S)+4 \pi r^{2} \gamma
$$

where $V$ stands for the volume and $r$ stands for the radius of the nuclei, $k_{\mathrm{B}}$ is the Boltzmann constant, $S$ and $\gamma$ are the saturation ratio and unit surface free energy, respectively. The parameter $S$ is a key point to modulate the whole nucleation $-\frac{4}{V} \pi r^{3} k_{\mathrm{B}} T \ln (S)$ process. As it is related to the volume exchange in solution, the higher saturation is helpful for the decrease of $G$ value. As such, the whole system tends to generate the smaller nuclei. By setting $\mathrm{d} \Delta G / \mathrm{d} r=0$, the critical radius of the nuclei $r\left(r^{*}\right)$ can be calculated as

$$
r^{*}=\frac{2 V \gamma}{3 k_{\mathrm{B}} T \ln (S)} .
$$

It is easy to find that the more saturate the solution is, the smaller the nucleus is, which is also beneficial for the nucleation process. This mode can perfectly explain the decomposition route of metal NCs reduction, during which the concentration of metal atoms continually increase with the decomposition of metal salts. As shown in Fig. 1a, the nuclei will form once the concentration of metal atoms reaches the point of supersaturation and the nucleation process will not stop until the equilibrium of generation of metal atoms from decomposition of metal salts and the consumption to give the nuclei. When the concentration of metal atoms is below the minimum supersaturation, the metal atoms cannot be supplied by the decomposition of metal salts and the nuclei will readily grow to form NCs, which can be defined as the growth step. From the viewpoint of thermodynamic description, this classical nucleation theory can also be understood by the free energy change $(\Delta G)$. The sum of the $\Delta G$ can be divided to $\Delta G_{\mathrm{v}}$ and $\Delta G_{\mathrm{s}}$, which are related to the phase transformation and the formation of new surface in the solution system. As the liquid is less stable than the solid one, the $\Delta G_{\mathrm{v}}$ is negative in the crystallization from solution and facilitates the nucleation. In contrast, the $\Delta G_{s}$ is positive due to the new interface of solid/liquid and benefits for the dissolution. Fig. $1 \mathrm{~b}$ illustrates the competition between the decrease in $\Delta G_{\mathrm{v}}$ and increase in $\Delta G_{\mathrm{s}}$ in the classical nucleation process. So, the rate of generation of nuclei generated from each unit volume can be defined as in the Arrhenius reaction rate equation:

$$
J=A \exp \left(-\frac{\Delta G_{\text {cit }}}{k_{\mathrm{B}} T}\right)
$$

where $A$ is the pre-exponential factor which is strictly related to the molecular mobility and temperature [21].

Though various nucleation processes can follow this classical nucleation theory, a number of researches have been conducted to reveal the differences between theoretical predictions and experimental results. That is, the classical theory usually overlooks the aggregation between two performed clusters and assumes all the clusters are only from the attachment of precursors. It is well known that many crystals can originate from successive combination of preassembled growth units such as nuclei, and clusters, rather than just from the monomer precursor. So, the nucleation process from the solution to generate nuclei may follow more complex routes rather than the only classical pathway. Wolde and Frenkel [23] adopted the Monte Carlo technique to study the nucleation process in a Lennard-Jones system and reported a two-step mechanism. In some systems such as nucleation of polymers or proteins (Fig. 1c), it is suggested that the formation of a liquid-like micelle will cause a large density fluctuations around the fluid-fluid critical point, which is not similar to the classical viewpoint. A crystalline nucleus beyond a certain critical size will form inside a highly disordered liquid droplet $[24,25]$. Together with the computational simulations, density functional theory (DFT) also evidences that this two-step nucleation will change the nucleation rate by several orders of magnitude [26]. Myerson and co-workers 

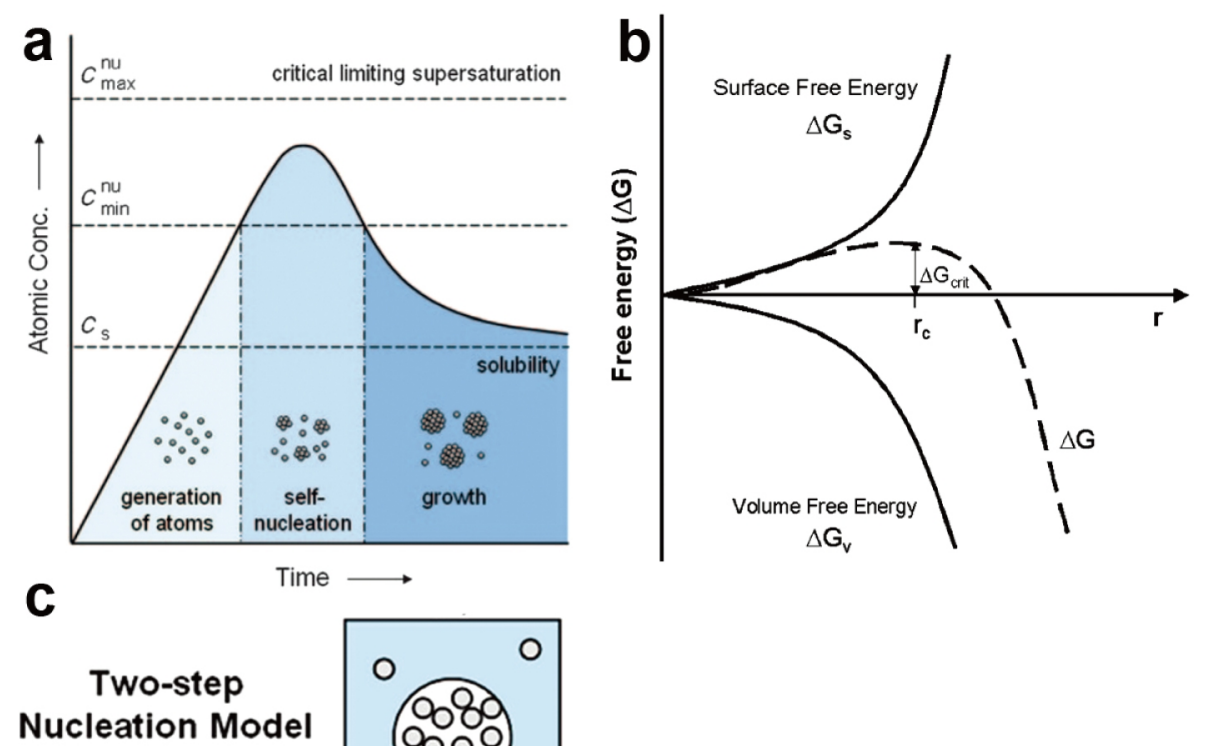

(a)

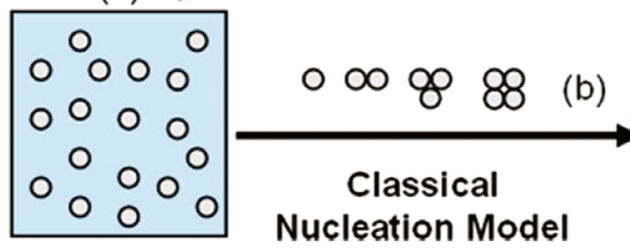

(d)

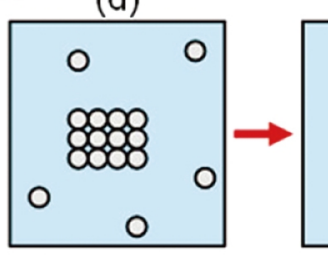

(e)

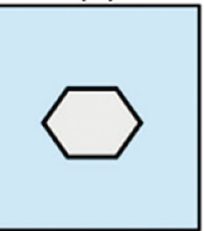

Figure 1 (a) Plot of atomic concentration against time, illustrating the classical nucleation process and subsequent growth. Reproduced with permission from Ref. [2], Copyright 2009, WILEY-VCH Verlag GmbH \& Co. (b) Free energy diagram for classical nucleation process. (c) Alternative pathways leading from solution to solid crystal. Reproduced with permission from Ref. [22], Copyright 2009, American Chemical Society.

[22] summarized a sophisticated review on nucleation theory of crystallization in solution.

\section{Growth process}

The above mentioned is related to the homogenous nucleation which happens without a solid-liquid interface. Actually, the growth process can be defined as the continual attachment of monomers to the preformed nuclei and seeds or the aggregation between nuclei. As a result, the specific parameters, such as lattice mismatch, crystal phase, exposed facets, capping agent, interparticle interaction, should be considered in the growth process [27-35]. Furthermore, when the size of nuclei or seeds is beyond the nanoscale, some colloidal behavior will also take place. As mentioned earlier, it is the competition between decreasing the bulk energy (which favors growth) and increasing surface energy (which favors dissolution) that directs the growth process. Though the structure of seeds to final $\mathrm{NCs}$ at different stages can be captured by various ex-situ characterization techniques, the observation of the growth at atomic level and the in-situ process that a monomer attaches on the seeds are very challenging, especially in the solution. To date, elucidating the real process how the seeds evolve into the NCs and which mechanism or principles the growth process will follow is still a great challenge. Thanks to the fast-developing in-situ characterization techniques with excellent spatial and temporal resolution, we are going to unravel the intrinsic nature during the synthesis of nanomaterials. Recently, we have summarized the latest advancement of the in-situ techniques in capturing the nucleation and growth process [36].

\section{Anisotropic growth}

Crystallization process can be defined as the supraatomic and supramolecular buildup in a highly ordered structure, during which the energetic and kinetic factors and transport of mass and heat will apparently affect the macroscopic morphology and real structures [37]. Considering the unit cell of NPs, anisotropic growth is common for the crystal growth, which is strictly dependent on the growth rate of each direction, epitaxial growth of two interfaces, and so on. In solution, this dynamic process 
proceeds heterogeneously, during which the interactions with solid items will reduce the activation energy. Actually, the growth rate along different directions usually derives from distinct atomic arrangement at relative facets. In an imaginary situation, the slow growing facets will prevail to reserve at a continuous crystal growth process. In contrast, the faster growing ones will result in the elongation of the adjacent facets and eventually disappear. The anisotropic growth is very common for the synthesis of semiconductor materials such as CdS [38], CdSe [39], CdTe [40], MnS [41] and $\mathrm{ZnO}$ [42]. Using the bottom-up strategy, the highly branched structures can be successfully built up through controlled nucleation of a zinc blende-phased core and following growth of wurtzite-phased pods. The synthesis is based on the intrinsic anisotropic nature of hexagonal cell structures of semiconductors along the $c$-axis.

For the highly symmetric face-centered cubic (fcc) metals, the construction of anisotropic structures in isotropic solution is quite challenging. Yang's group reported the shape evolution from cube through cuboctahedron and finally to octahedron. This phenomenon originates from the competition between the growth of $\{111\}[46,47]$ and $\{100\}$ facets [43]. Actually, the success of controlling the Ag nanocube and Ag octahedron with well-defined size and shape can be also achieved by starting with Ag nanospheres [48]. Significantly, the Ag octahedra can be well controlled with small sizes, regular and sharp corner/edges and welldefined facets through the binding of citrate on $\mathrm{Ag}$ surface. Later, the competition between the growth of different crystal facets was also reported in the synthesis of concave Ag NCs such as octahedron, cube, octapod and trisoctahedron (Fig. 2b) [44]. In addition, some metal NCs with high-index facets and unconventional shapes [5] can be also generated by sophisticated control. A breakthrough was gained by Sun, Wang and co-workers, during which a unique tetrahexhedral Pt NC was obtained by square-wave potential [45] (Fig. 2c). These Pt NCs are 750-nm diameter polycrystalline, $O_{h}$ symmetry, and bounded by 24 high-index planes of $\{h k 0\}$ [49-57], which can be considered as six square-based pyramid covering a cube in the center. Besides Ag and $\mathrm{Pt}$, the controlling of facets by manipulating the anisotropic growth of fcc metals can be also extended to Pd based NCs [58], and so on. Usually, the common exposed facets of fcc metals are $\{111\}$ and $\{100\}$ facets with low percentage of corner and edge atoms. Because the surface energy tends to minimize to the lowest, the high-index facets $\{h k l\}$ with at least one $h, k$, and $l$ equal to two or greater are difficult to be obtained (Fig. 2d) [5]. So, the preparation of NCs that are enclosed with high-index facets requires more accurate control of anisotropic growth of each facet. In addition, some highly branched structures with irregular morphologies can be also prepared by choosing the right capping agent and experimental condition for the anisotropic growth. For example, the preparation of hyperbranched polyvillic Au NPs was recently reported by Pan and co-workers through an anisotropic propagation technique [59]. These shape evolutions in the growth steps can also be recognized as seed growth or over growth, which will be discussed afterwards [60].

\section{Capping agent}

In general, the capping agents are composed of counter ions (e.g., halide ions) in the precursors, solvents (e.g., $\mathrm{N}, \mathrm{N}$-dimethylformamide), salts, surfactants (e.g., amines, carboxylic acids, phosphines), polymers (e.g., polyvinyl pyrrolidone (PVP), polyvinyl alcohol), gas molecules (e.g., CO), and so on. Among these chemicals, polymers, surfactants, and small adsorbates are three major types of capping agents for the synthesis of NCs due to their strong effect on dictating the morphology. Wang and co-workers [61] reported that polyoxometalate can serve as the capping agent to tune the growth of metal-organic frameworks (MOFs). The reason of shape modulation is based on the strong interaction between anodes of MOFs and polyoxometalate anions. Our group recently have given a comprehensive review about the classification of capping agents on NCs' surface, capping agents in colloidal synthesis, and capping agents on catalysis [62].

In the solution system for synthesis of NCs, the capping agents can serve as versatile roles rather than only being the stabilizers. As well known, the coordination number of atoms varies between the surface and bulk. Moreover, the coordination number even varies with different exposed facets due to the surface atoms arrangement. For fcc metals which adopt the $\mathrm{ABCABC}$ stacking sequence along $\{111\}$ directions, the coordination number of the so-called lowindex facets such as $\{111\},\{110\}$ and $\{100\}$ is 9,8 , and 7 , respectively, which are not like that of the bulk atoms (12). So, the surface energies increase as the order of $\gamma\{111\}<$ $\gamma\{100\}<<\{110\}$. As Miller called $\{h k l\}$ surface with at least one index over 1 the high-index facets, these facets owning high density of low-coordination atoms and increased surface energy, thus are generally difficult to be prepared. One important role of the capping agents is to effectively lower the surface energy of NCs. The surface energy of specific facets can be largely decreased if the selected capping agents can effectively binds to their surface. If we want to expose the specific facets, the most crucial factor is how to choose 

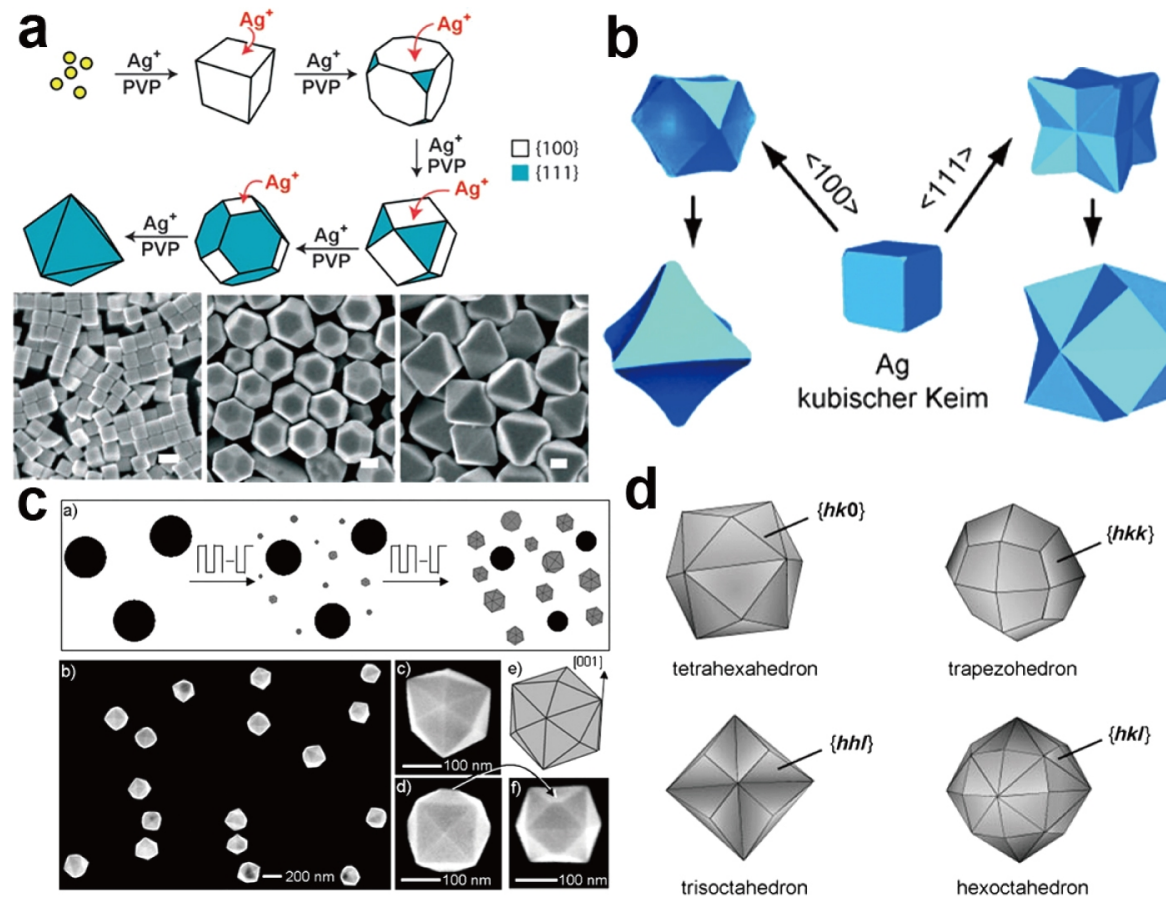

Figure 2 (a) The top is the shape evolution from Ag cube to octahedron. The bottoms are the representative transmission electron microscopy (TEM) images. Reproduced with permission from Ref. [43], Copyright 2006, WILEY-VCH Verlag GmbH \& Co. (b) Scheme illustrating the Ag NCs with concave surfaces from the Ag cubic seeds. Reproduced with permission from Ref. [44], Copyright 2011, WILEY-VCH Verlag GmbH \& Co. (c) Pt THH NCs. Reproduced with permission from Ref. [45], Copyright 2007, American Association for the Advancement of Science. (d) Unconventional shapes of fcc metals whose surface is enclosed by high-index facets. Reproduced with permission from Ref. [5], Copyright 2007, WILEY-VCH Verlag GmbH \& Co.

the right capping agents to bind on and make them the lowest surface free energy.

Another function that capping agent can bring into the synthesis is modulating the growth rate on certain facets. If a set of facets are tightly covered by the capping agents, fewer free sites for the deposition of atoms are left. As the facets with higher growth rates diminish, the facets depressed by the capping agents will be reserved in the final NCs. Indeed, the modulations from the capping agents involve both the thermodynamic control (the selective binding to decrease surface energy) and the kinetic control (varying the specific growth rate). The fast growth stage with a low temperature and high degree of saturation will follow the kinetic regime, and the final morphology is strictly related to the growth rate over different facets [66]. By contrast, the growth stage with high temperature and low growth rate will result in the facet owning the lower surface energy. These two regimes are not independent during the growth though the contributions depend on the experimental conditions. In the overwhelming majority of cases, the trends of these two effects are the same. As a result, researchers discovered many specific capping agents that can selectively dictate the exposed facets. Xia and co-workers [58,67] reported the oxygen atoms of PVP can selectively bind to the $\{100\}$ facets of $\mathrm{Ag}$ and $\mathrm{Pd}$ NCs. Cetyltrimethylammonium bromide (CTAB), as a cationic surfactants, can facilitate the controlled synthesis of one-dimensional (1D) Au structures [68]. Based on this classical reactive system, the Au nanorods with different length-diameter ratios can be successfully induced $[69,70]$ and applied to the surface enhanced Raman scattering (SERS) [71], cellular imaging [72], photothermal therapy $[73,74]$, and so on due to its excellent plasmonic properties [75,76]. As $\mathrm{Br}^{-}$was firstly reported by Xiong et al. [77] to play an important role in tuning the Pd nanocubes and nanobars, the halide ions have been widely used in controlled synthesis of Pd [78-80], $\mathrm{Pt}$ [81], Rh [82,83] NCs and so on. Yin et al. [84] reported the remarkable role of $\mathrm{C}_{2} \mathrm{O}_{4}{ }^{2-}$ in the controlled synthesis of Ru NCs. For gas molecules, Huang et al. [63] reported that the increased concentration of $\mathrm{NO}_{2}$ can facilitate the stabilization of $\mathrm{Pd}\{111\}$. Zheng's group [8] reported that $\mathrm{CO}$ was a preferential capping agent to adsorb on $\operatorname{Pd}\{111\}$ and demonstrated its successful utilization in the preparation of Pd nanosheets (Fig. 3a). They also denoted a detailed review on how to utilize the small adsorbate to achieve shape control of Pd and Pt NCs. 

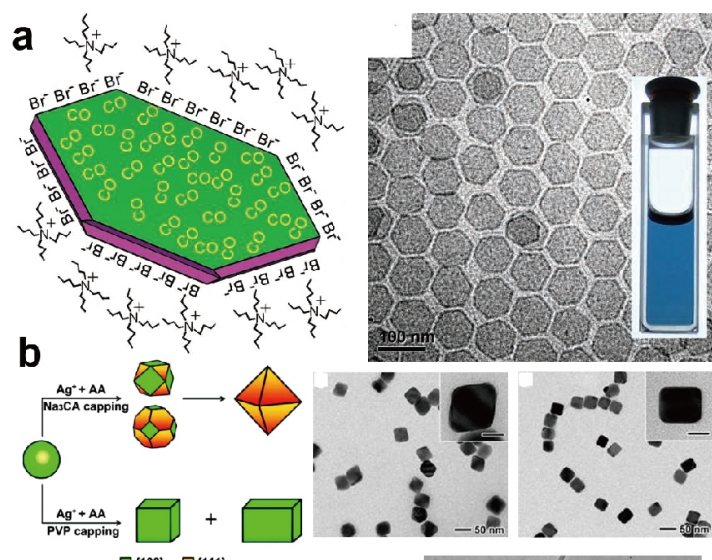

C
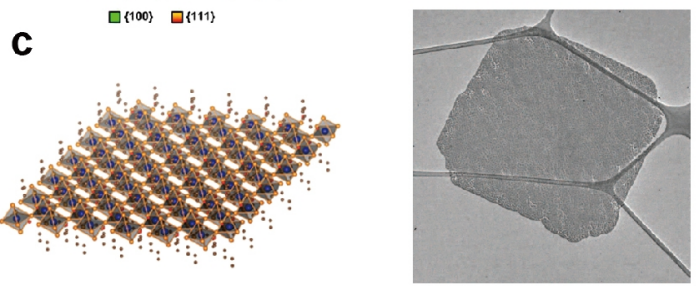

Figure 3 (a) Structure model of a hexagonal Pd nanosheets with $\mathrm{CO}$ molecules on the $\{111\}$ facets, $\mathrm{Br}^{-}$on the side $\{100\}$ facets and $\mathrm{NBu}_{4}{ }^{+}$as the counter ions. The right is the TEM image of ultrathin Pd nanosheets. Reproduced with permission from Ref. [63], Copyright 2011, Nature Publishing group. (b) Scheme illustrating the anisotropic growth of $\mathrm{Ag}$ NCs induced by selective capping. Reproduced with permission from Ref. [64], Copyright 2012, American Chemical Society. (c) Structural illustration of a single layer $\left(\mathrm{C}_{4} \mathrm{H}_{9} \mathrm{NH}_{3}\right)_{2} \mathrm{PbBr}_{4}$ and the corresponding TEM image. Reproduced with permission from Ref. [65], Copyright 2015, American Association for the Advancement of Science.

$\mathrm{Xia}$ and co-workers [64] demonstrated the anisotropic growth of Ag NCs by the selection of capping agents. For bimetallic NCs, the selection of capping agents should consider both of the two metals on their surface. Tao's group [85] gave a review about the commonly used facet-specific capping agents and their preferential binding facets in the shape control of bimetallic NCs through well-designed colloidal chemistry approaches. Most recently, Yang's group [65] showed how to use the layer of butylammonium to induce the formation of atomically thin $2 \mathrm{D}$ organic-inorganic hybrid perovskites (Fig. 3c).

\section{Interparticle interaction}

In this section, we mainly talk about the interparticle interaction. For this case, most of the monomers are consumed in the nucleation process, and the coalescence and dissolution between crystallites driven by thermodynamic or kinetic factors dominate in this growth process.

In crystal systems, the nucleation process can be generally considered to be attachment and of free nuclei. For the growth stage, driven by the demand for reducing overall surface energy, the attachment between two or more NCs is common. If the attachment undergos an oriented fashion, during which adjacent particles share a common crystallographic orientation and join together as a planer interface, this process can be called as oriented attachment. In this coalescence-based NC growth, the surface energy is reduced by eliminating pairs of surfaces, resulting in a strong thermodynamic driving force. Utilizing this coarsening mechanism containing oriented attachment followed by growth can effectively achieve the control of single crystal, defects, twinning, stacking faults and polytypic intergrowth [89-92]. Oriented attachment provides special benefit for synthesizing single crystal 1D materials such as nanowires, nanorods, and so on. Driven by the oriented attachment, the particles tend to fuse with same crystallographic facets and generate defect-free single crystals. For classical examples, Murray and co-workers [93] reported how to generate single-crystal $\mathrm{PbSe}$ nanowires by oriented attachment of single nanodots in solution. Hyeon and co-authors [86] reported the synthesis of quantum-scale $\mathrm{ZnS}$ nanorods by oriented attachment mechanism (Fig. 4a). Further, the shape of $\mathrm{ZnS}$ can be easily tuned by altering the experimental parameters. Ravishankar's group [94] gained the pioneering work of preparing ultrafine single-
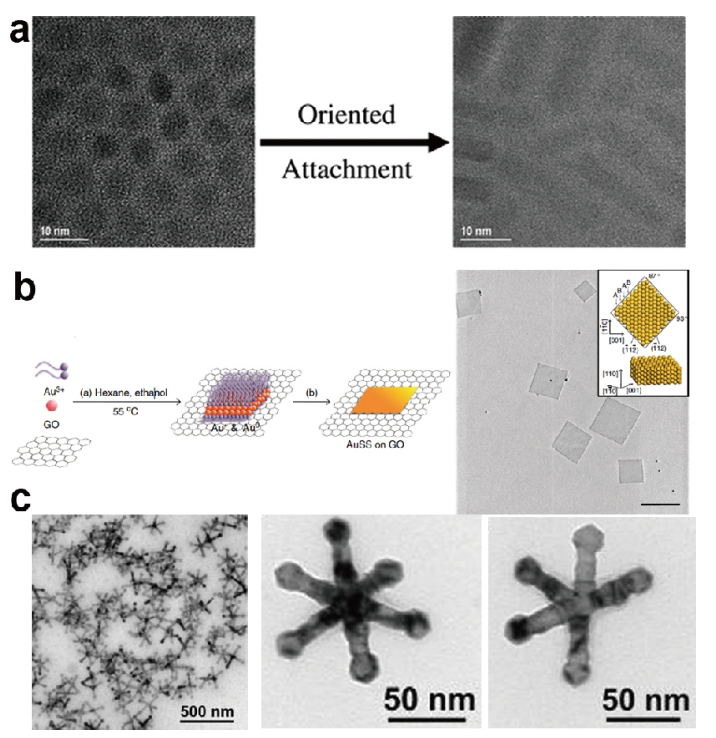

Figure 4 (a) Structure model and TEM images of the formation of $\mathrm{ZnS}$ nanorods by oriented attachment. Reproduced with permission from Ref. [86], Copyright 2011, American Chemical Society. (b) Scheme and TEM image illustrating the anisotropic growth of Au nanosheets. Adapted with permission from Ref. [87], Copyright 2011, Nature Publishing group. (c) An overview and magnified TEM images of MnO multipods. Reproduced with permission from Ref. [88], Copyright 2005, American Chemical Society. 
crystalline Au nanowire arrays by oriented attachment of NPs. Later, Yu's group [95] contributed a short review on the oriented attachment-mediated growth and synthesis of functional materials especially the 1D type. 2013, Xie's group [96] reviewed that how to use the oriented attachment strategy to obtain atomically thick $2 \mathrm{D}$ crystals. The small inorganic building blocks were arranged in a confined 2D space and adopted a similar crystallographic face by the thermodynamic driving force. Unlike the layered crystal structure owning the intrinsic trends, the $2 \mathrm{D}$ anisotropic growth of nonlayered structure is very difficult. As a result, oriented attachment is becoming an exclusive way and has been widely applied to a variety of atomically thick 2D crystals of non-layered materials such as $\mathrm{Co}_{9} \mathrm{Se}_{8}$ [97], PbS [98], Au (Fig. 4b) [87], Rh [99], and so on. Actually, the oriented attachment can also be utilized to build three-dimentional (3D) nanostructures such as branched structures. One interesting example was reported by Belin's group [88] in the synthesis of manganese oxide. During their synthesis, single-crystalline $\mathrm{MnO}$ multipods were synthesized by single-step synthesis. The preparation was based on the thermal decomposition of $\mathrm{Mn}$ (oleate) $)_{2}$ and oriented attachment of $\mathrm{MnO}$ small NPs (Fig. 4c). In 2010, Xia and Lim [3] published a comprehensive review of highly branched metal structures, in which oriented attachment was an important strategy. Recently, $\mathrm{Hu}$ and Wang [100] reported a review about how to control the synthesis of ultrathin nanostructures, in which oriented attachment also plays an important role. No matter 1D, 2D or 3D gesture the building blocks adopt, the thus-formed nanostructures will typically retain at least the original size scale in 1D. Considering that the building blocks are mainly clusters and small crystal that can be dubbed as "ultrathin unit", the oriented attachment exhibits excellent advantages in tailoring the ultrathin structures.

\section{Underpotential deposition}

During the past decades, the underpotential deposition (UPD) has been discovered to vary the deposition onset of metal atoms at pre-formed substrates under a more positive reduction potential versus standard Nernst potential $[47,101]$. The analysis of UPD can indeed deepen the understanding of monolayer formation of metal species on different single-crystal surface or polycrystalline that can exhibit distinct catalytic behaviors. At a fundamental level, Sudha and Sangaranarayanan [102] demonstrated the dependence of UPD on work function differences, lattice mismatch, solvent desorption energies, and surface coverages. They also indicated that the formation of monolayer was strictly governed by transport process which was incorporated with bulk deposition. The existence of UPD was confirmed by cyclic voltammetric studies, during which peaks corresponding to monolayer deposition can be observed at more positive potentials than Nernst potential. Because the UPD of monolayer metal on the substrates can largely affect the geometrical and electrical structures, the work functions will be varied by the depositions. Actually, the variation of total Gibbs free energy can be traced during the UPD process, which is imperative to involve various processes contributing to the overall potentials. The UPD of monolayer of $\mathrm{M}$ on $\mathrm{S}$ can be divided as: (1) the movement of free ions solvated in solution to reaction zone $\Delta G_{\mathrm{I}}$, (2) electron transfer from substrate to metal ions $\Delta G_{\text {III }}$, and (3) desorption of solvent dipoles and formation of metal-substrate (S-M) bond $\Delta G_{\text {III. }}$ So, the change of total Gibbs free energy can be regarded as:

$$
\Delta G_{\mathrm{ML}}=\Delta G_{\mathrm{I}}+\Delta G_{\mathrm{II}}+\Delta G_{\mathrm{III}} .
$$

Scheme 2 indicates that the UPD process can be recognized as being dictated by crystal imperfections and knocking off the adsorbed solvent dipoles from specific sites of the substrate [102].

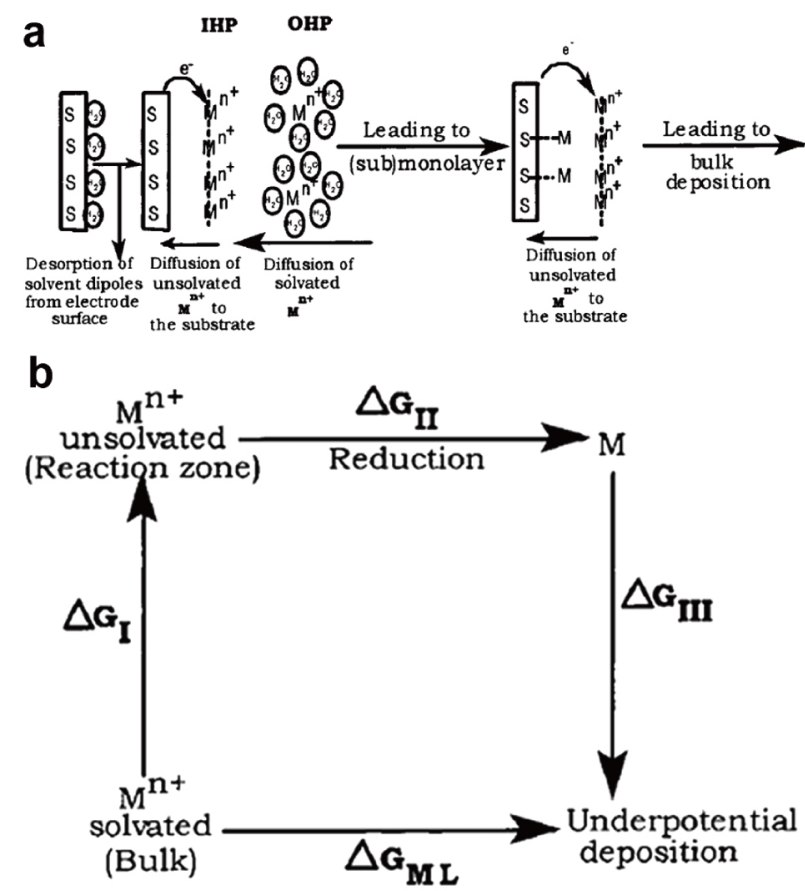

Scheme 2 (a) Schematic representation of UPD process of monolayer $M$ on the substrate (S). (b) Thermochemical cycle denoting different energetic contributions involved in the monolayer. Reproduced with permission from Ref. [102], Copyright 2002, American Chemical Society. 
The UPD mechanism has been successfully adopted to construct abundant nanomaterials. Mirkin and colleagues [104] reported that well-defined Au nanostructures with four morphologies such as octahedra, rhombic dodecahedra, truncated ditetragonal prisms, and concave cubes, can be selectively synthesized by a seed-mediated growth strategy (Fig. 5). During this synthesis, the shapes directly depend on the concentration of $\mathrm{Ag}^{+}$in the reaction solution. The X-ray photoelectron spectroscopy and inductively coupled plasma atomic emission spectroscopy analyses quantitative evidenced the trace Ag deposited on the $\mathrm{Au}$ surface and explained the modulation of various enclosed surface facets. The concentration of $\mathrm{Ag}^{+}$can effectively affect the coverage of $\mathrm{Ag}^{+}$and its gesture adsorbed on the $\mathrm{Au}$ surface. As a result, increasing the concentration of $\mathrm{Ag}^{+}$ would finally result in more open surface facets, as expected via the calculated UPD monolayer of Ag. Afterwards, Xie's group [105] discovered that the UPD mechanism can also be applied to the bimetallic surface. In detail, the addition of $\mathrm{Cu}^{2+}$ would assist the formation of hexotachedral Au-Pd alloy NCs with high-index facets. Abruna and co-workers [106] reviewed the UPD mechanism at single crystal sur-
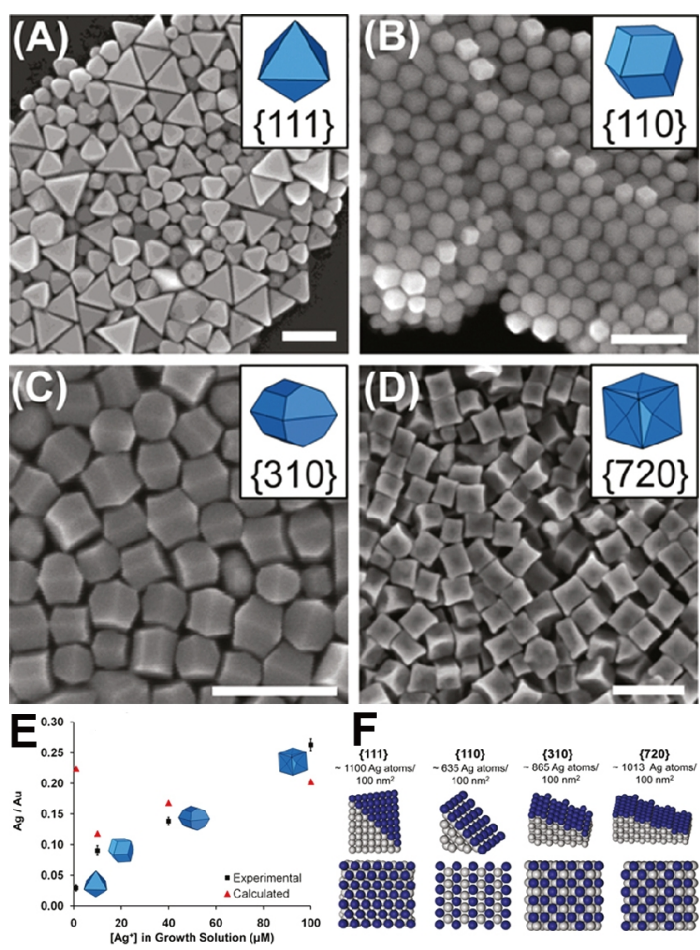

Figure 5 (a-d) SEM images of Au NCs with different shapes obained by UPD of monolayer of Ag. (e) Ag/Au ratio for each particle type. (f) Maximum number of surface atoms per unit area, side view, and top view models for each of the four reported surface facets. Reproduced with permission from Ref. [104], Copyright 2005, American Chemical Society. face of $\mathrm{Au}, \mathrm{Pt}, \mathrm{Ag}$, and other materials. Although the original UPD of metal monolayer has been proved to indeed assist seed growth procedure for further modifications of substrate and has continuously expanded accessing to anisotropic metal NPs, the direct experimental evidence of metal monolayer remains difficult to obtain. Due to the difficulty in imaging and detecting the spatial distribution of metal monolayer on substrate, the understanding of UPD mechanism is still challenging. Wright and co-workers [107] presented the first experimental evidence on the position of $\mathrm{Ag}$ on $\mathrm{Au}$ nanorods by using a Tecnai Osiris equipped with a Super-X EDS energy dispersive X-ray spectroscopy (EDS) system. Simultaneous X-ray detection of four solid-state detectors in conjunction with a high brightness Schottky-type field emission gun can allow the element-specific mapping at subnanometer resolution across the periodic table with high sensitivity and high spectral count rates. Most recently, our group synthesized the monolayer Ru atoms covered highly ordered porous $\mathrm{Pd}$ octahedra via the UPD and thermodynamic control [108]. Shape evolution from concave nanocube to octahedron with six hollow cavities was observed due to the UPD of monolayer Ru. Quantitative evidence was provided by aberration corrected high-resolution transmission electron microscopy (HRTEM) and X-ray photoelectron spectroscopy (XPS) which proved that only a monolayer of $\mathrm{Ru}$ atoms was deposited on the surface.

\section{Phase evolution}

In recent years, apart from the size, shape and composition, the crystal phase has also been reported as an important factor in dictating the functionality and properties (e.g., chemical reactivity and stability, magnetic, optical, mechanical, and catalytic behaviors) of nanomaterials. Therefore, the control of crystal phase during the nucleation and growth process has emerged as a matter of concern for the investigation of synthetic methodologies. For example, the metals such as $\mathrm{Au}, \mathrm{Ag}, \mathrm{Pd}, \mathrm{Pt}$, usually crystallize as fcc structure. In contrast, when their sizes are down to nanoscale, the proportion of surface atoms significantly increases and the surface energy may largely affect the total systemic energy. Therefore, some unusual phase may form during the nucleation or growth stages. Sun et al. [109] observed the crystal phase evolution of Fe-Pt alloys from $\mathrm{fcc}$ to face-centered tetragonal (fct) at high temperature during the synthesis. Afterwards, under controlled synthetic conditions, hexagonal close-packed (hcp, $2 \mathrm{H}$ type) and $4 \mathrm{H} \mathrm{Au},[87,110]$ body-centered tetragonal (bct) $\mathrm{Ag}$ [111], fcc Fe [112], hcp Ni [113], fcc Ru [114] and hcp 
Rh [99] nanostructures can be prepared. In addition, during some typical growth stages such as seed growth, ligand exchange, and metal coating, the unusual phase or phase evolution can be also observed. Wei and co-workers [115] demonstrated the icosahedral-to-cuboctahedral structural transformation of $\mathrm{Au}$ nanoclusters can be achieved by varying the surface ligands. In detail, the icosahedral $\mathrm{Au}$ clusters capped by dodecanethiol and triphenylphosphine can be transferred into cuboctahedral structure by the desorption of surface ligands and structural rearrangement of $\mathrm{Au}$ atoms in different chemical environments (Fig. $6 a)$. This work addressed that the surface structure could largely affect the dramatic change in the whole architecture if the size was reduced to some extent. Zhang's group [116] recently devoted a lot of efforts in studying the rare metal phases during the aforementioned processes. In detail, the epitaxial of Pt on hcp Au square sheets can directly dictate the phase transformation of Au nanosheets from hcp to fcc under mild conditions. (Fig. 6b) They also reported that the surface ligand-exchange from oleylamine to octadecanethiol can drive the phase evolution from hcp Au nanosheet to fcc structure [46]. Similarly in bimetallic and trimetallic system, Sun and co-workers [117] presented how to control the chemically ordered fct PtFeAu and PtFe by varying the synthetic conditions such as reactive temperature. The activity and durability towards the electro-oxidation of formic acid can be further improved by this phase control. Most recently, Yao et al. [118] reported an epitaxial-growth-mediated method to grow fcc $\mathrm{Ru}$, which was thermodynamically unfavorable in the bulk form, on the surface of Pd-Cu alloy (Fig. 6c). The moderate lattice mismatch between the overlayer and the substrate indeed played a crucial role in dictating the successful coating of the unconventional crystallographic structure. Overall, recent studies have revealed a clear clue that the crystal phase evolution can take place during some nucleation and growth processes for preparation of nanomaterials. In addition, some chemical and physical properties such as chemical stability, and magnetic, electrical and optical properties can be also influenced by the control over the crystal phase of nanostructures. Recently, Fan et al. [119] contributed a comprehensive review on crystal phase-controlled synthesis, properties and applications of noble metal nanomaterials, which could provide beneficial reference for interested readers.

\section{BOTTOM-UP STRATEGY}

The bottom-up strategies basically start with the reaction between precursor monomers such as metal ions, clusters, metal complex, and so on. This method may provide a useful route to atomically precise fabrication of nanomaterials based on the rational design of precursor monomer and the
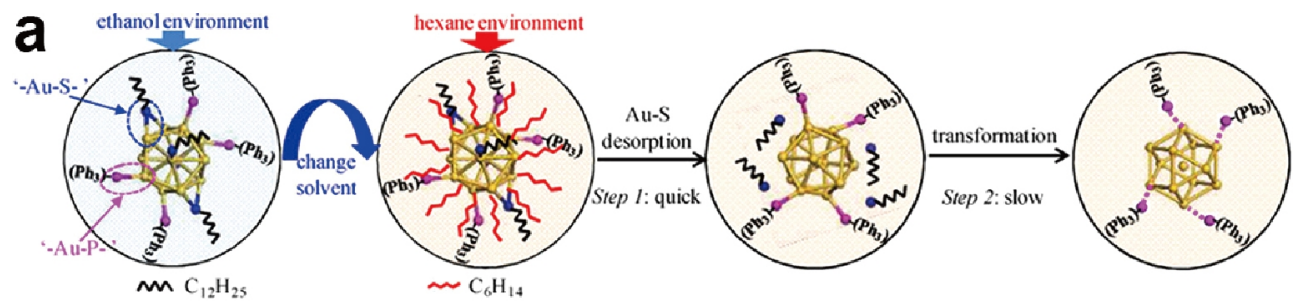

$\mathrm{Au}_{13}\left(\mathrm{PPh}_{3}\right)_{4}\left(\mathrm{SC}_{12} \mathrm{H}_{25}\right)_{4}\left(l_{h}\right)$

b
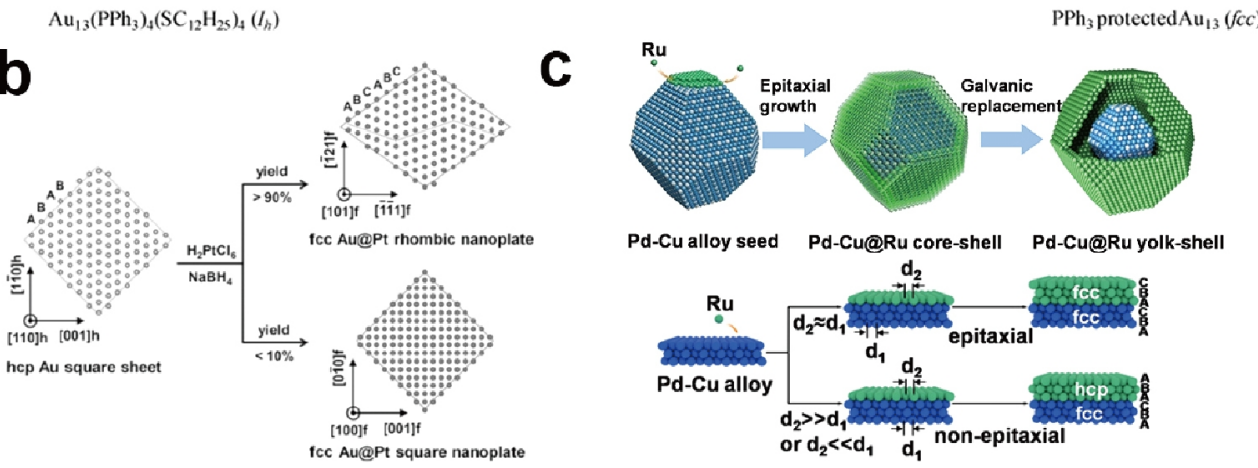

Figure 6 (a) Schematic illustration of the two-step process of the structural transformation. Reproduced with permission from Ref. [115], Copyright 2012, American Chemical Society. (b) Schematic illustration of the synthesis of (101) f-oriented fcc Au@Pt rhombic nanoplates with twinning (marked by the dashed line) from hcp Au square sheets (top panel). A small amount of $(100)_{\mathrm{f}}$ oriented fcc Au@Pt square nanoplates (bottom panel) were also formed. Reproduced with permission from Ref. [116], Copyright 2015, WILEY-VCH Verlag GmbH \& Co. (c) Schematic illustrations of the formation of fcc Ru by the epitaxial growth on fcc Pd-Cu substrate. Reproduced with permission from Ref. [118], Copyright 2016, WILEY-VCH Verlag GmbH \& Co. 
manipulation of the bottom-up reactions. For example, Langille et al. [120] reported a bottom-up strategy to generate Au octahedra with tailorable hollow features. For this synthesis, the UPD of Ag on the Au surface is also critical points for the control of the hollow dimensions and size of hollow cavities. Xia and co-workers [121] reported the spherical colloids of low melting-point metals can be separately prepared both from the bottom-up and top-down approaches. The bottom-up production of monodispersed bismuth particles is thermally decomposed from bismuth acetate in boiling ethylene glycol (EG). In contrast, the topdown approach is based on the emulsifying molten drops of bismuth in boiling di(ethylene glycol) and quenching with cold ethanol. Actually, the bottom-up strategy is a common statement in the synthesis of carbon-based materials such as graphene. For example, Sun's group [122] reported the defluorination of polymer can be achieved by the catalysis of $\mathrm{CaC}_{2}$ to fabricate crumpled graphene through bottom-up strategy. Due to the wide range of precursors, the topology, size, composition, structures of nanomaterials can be defined by the right selection and design. In the following, we will discuss three representative reactions of precipitation, reduction and decomposition.

\section{Precipitation reaction}

In the field of chemical reaction, precipitation reaction is one of the most basic bottom-up reactions. It is well known that, in solution systems, monoatomic cations such as metal ions can undergo precipitation reactions with polyatomic anions or monatomic anions. To date, the cations used in the precipitation route mainly contain the metallic elements. The polyatomic anions used involved the $\mathrm{OH}^{-}$, $\mathrm{WO}_{4}{ }^{2-}, \mathrm{PO}_{4}{ }^{2-}$, and so on, and the monatomic anions used mainly include chalcogen ions $\left(\mathrm{S}_{2}^{-}, \mathrm{Se}_{2}{ }^{-}\right.$, etc. $)$and halogen $\left(\mathrm{F}^{-}, \mathrm{Cl}^{-}, \mathrm{Br}^{-}, \mathrm{I}^{-}\right)$ions. One important classication for precipitation is the complex derived from the coordination between anodes and ligands such as MOFs. As a result, this reaction has been used for synthesizing a variety of solid nanomaterials such as semiconductors, hydroxides, oxides, inorganic salts, MOFs, and so on. In solution, solubility product $\left(K_{\mathrm{sp}}\right)$ is the most key factor to precipitation reaction which can directly trigger the reaction. Through the precipitation process between metal ions and chalcogen ions, most chalcogenide can be obtained. Our group recently contributed a review about the controlled synthesis of semiconductor nanostructures by solution-based methods [123]. Afterwards, Eychmuller and co-workers [124] also discussed the synthesis of colloidal semiconductor in aqueous system, during which the chemical structures of widely used thiol capping ligands were listed in detail. Kershaw et al. [125] contributed a review about the synthetic methods for narrow bandgap colloidal metal chalcogenide quantum dots and their heterostructures, assemblies, together with their corresponding electronic and infrared optical properties. In 2013, Yu's group [126] mentioned the synthesis, modification, energy conversion, and storage devices of nanostructured metal chalcogenides. The synthetic methods involved in the chemical precipitation are always based on traditional colloidal chemistry, during which metal cations react with inorganic chalcogenide source to form a new phase in the solution. Since Ekimov and Brus $[127,128]$ discovered the quantum confinement effects that the optical properties are strictly related to their size, the size control of semiconductors is very important for the synthesis of semiconductor NCs.

As typical instances, Henglein and co-workers achieved the synthesis of monodispersed CdS NCs through the reaction between $\mathrm{Cd}^{2+}$ and $\mathrm{H}_{2} \mathrm{~S}$ under well-defined $\mathrm{pH}$. Li et al. [129] have also achieved the controllable synthesis of the well-monodisperse copper chalcogenide NCs by using $\mathrm{Cu}_{3} \mathrm{~N}$ NPs as copper precursors through a facile one-pot method, and the size and compositions of $\mathrm{CuS}$ can be readily controlled by varying the ratio of the precursors. Our group demonstrated the dodecanethiol can be used as the oil phase-ligands, solvent, reducing agent and also the sulfur source to give rise to high-quality monodispersed $\mathrm{Ag}_{2} \mathrm{~S}$ and $\mathrm{Cu}_{2} \mathrm{~S}$ [130] (Fig. 7a). Moreover, the shape of $\mathrm{Cu}_{2} \mathrm{~S}$ can be further altered by adding the anions such as $\mathrm{Ac}^{-}$ or $\mathrm{Cl}^{-}$. The semiconductor NCs obtained by this method exhibit narrow size distribution and can be self-assembled into a superlattice spontaneously [131] (Fig. 7c). In 2013, Zhao's group [132] reported a facile hydrothermal route for the synthesis of unique $3 \mathrm{D}$-open porous hierarchical $\mathrm{Cu}_{2} \mathrm{~S}$ microsponges by using cetyltrimethylammonium bromide as the morphology controlling agent. The obtained $\mathrm{Cu}_{2} \mathrm{~S}$ microsponges are constructed from $2 \mathrm{D}$ ultrathin $(1.5 \mathrm{~nm})$ single-crystal nanosheets with exposed (111) facets. Additional, Jiao et al. [133] reported well-defined mesocages copper sulfide using shape-controlled $\mathrm{Cu}_{2} \mathrm{O}$ crystal template. The authors used the difference between the $K_{\mathrm{sp}}$ of $\mathrm{Cu}_{2} \mathrm{O}$ and $\mathrm{Cu}_{2} \mathrm{~S}$ to form core-shell $\mathrm{Cu}_{2} \mathrm{O} / \mathrm{Cu}_{2} \mathrm{~S}$ in $\mathrm{Na}_{2} \mathrm{~S}$ aqueous solution of $\mathrm{Cu}_{2} \mathrm{O}$ as seeds. Then, the dissolution of the inner $\mathrm{Cu}_{2} \mathrm{O}$ cores in ammonia solution formed mesoporous copper sulfide cages. This strategy is also universal for other shape of $\mathrm{Cu}_{2} \mathrm{O}$. Akkerman et al. [134] developed binary $\mathrm{Cu}_{2} \mathrm{~S}$ to ternary $\mathrm{Cu}-\mathrm{In}-\mathrm{S}$ and quaternary $\mathrm{Cu}-\mathrm{In}-\mathrm{Zn}-\mathrm{S}$ system. They utilized different $K_{\mathrm{sp}}$ in $\mathrm{Cu}^{+}, \mathrm{In}^{3+}, \mathrm{Zn}^{2+}$ and $\mathrm{S}_{2}{ }^{-}$ to create a $\mathrm{ZnS}$-rich shell with the unchanged size and 


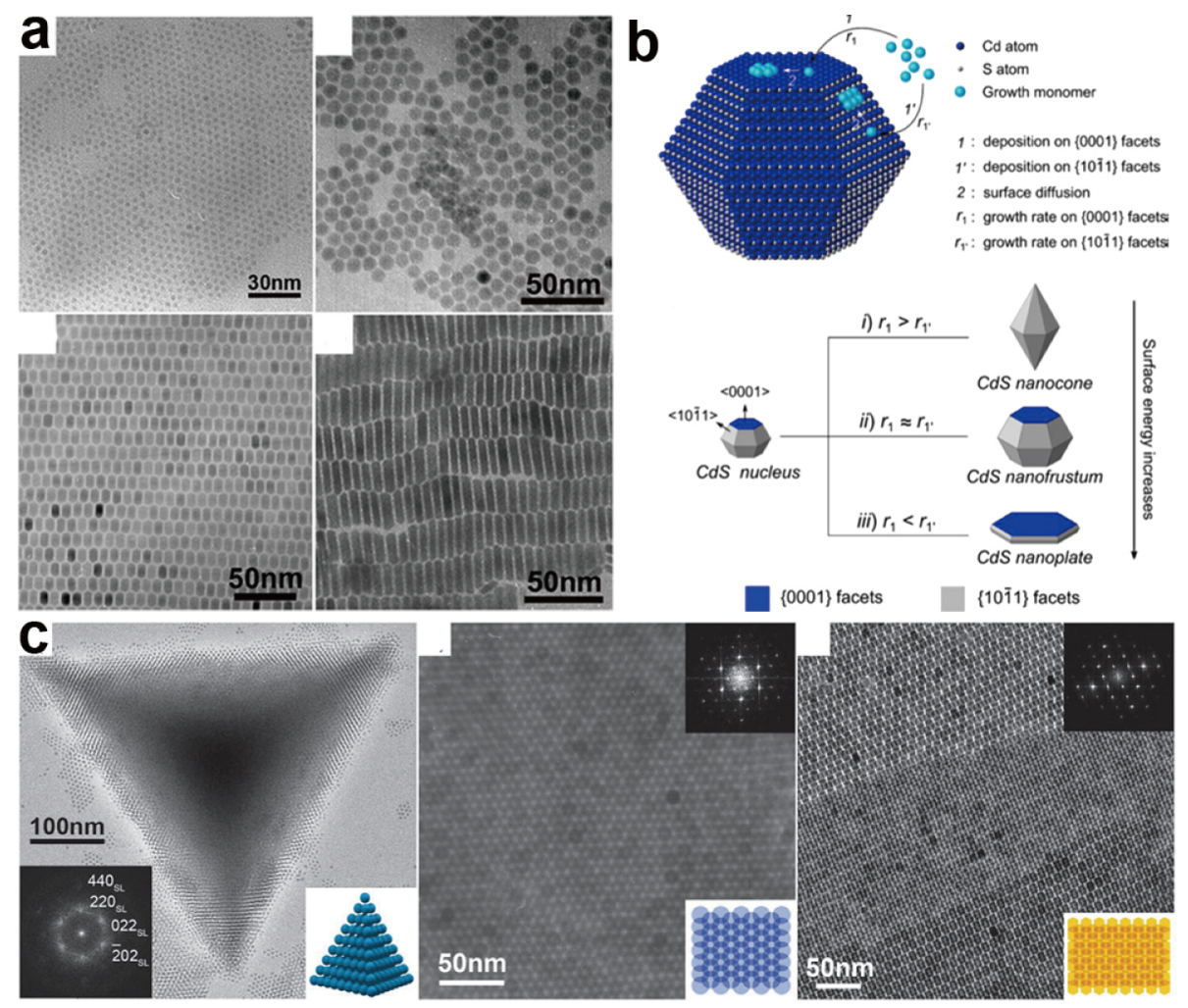

Figure 7 (a) NCs synthesized by the dodecanethiol-assisted two-phase interface confined method. Reproduced with permission from Ref. [123], Copyright 2011, Royal Society of Chemistry. (b) Schematic illustration of the major steps involved in the growth process and the formation of a CdS nanocone, a CdS nanofrustum, and a CdS nanoplate. Reproduced with permission from Ref. [136], Copyright 2015, American Chemical Society. (c) TEM images showing the self-assembly of NCs into 3D advanced structures. Reproduced with permission from Ref. [131], Copyright 2008, American Chemical Society.

shape. Afterwards, a more complex $\mathrm{CdS} / \mathrm{HgS} / \mathrm{CdS}$ coreshell structure prepared through a sequential precipitation process was reported by Weller and co-workers [135].

For the precipitation process, additional foreign stabilizing molecules are usually introduced to prevent the further growth and aggregation of the NCs. To control the exposed facets, Wang et al. [136] prepared (0001) facets and (1011) facets of CdS by simply employing a syringe pump, which enables us to finely tune the crystal shape from nanocones, to nanofrustums, and further to nanoplates (Fig. 7b). The growth was demonstrated to be kinetically diffusion-controlled. Changing the $\mathrm{Cd}^{2+}$ injection rate simply leads to the tunable shape evolution. Another method to grow metal sulfide is by means of seed growth. Recently, Ma and co-workers [137] reported a general and facile method to prepare homogeneous dumbbell-like gold-sulfide core-shell hetero-nanostructures. The evolution from $\mathrm{Au}$ nanorods to dumbbell-like Au nanorods and the coating of metal sulfide shells were achieved in a one-pot reaction (Fig. 8). Dutta and co-workers [138] reported a gel-based approach to design hierarchical $\mathrm{CuS}$ decorated reduced graphene oxide ( $\mathrm{r}-\mathrm{GO}$ ) nanosheets. They have gained the success in the fabrication of porous tubular copper sulfide nanostructures from $\mathrm{Cu}^{2+}$-thiourea on the r-GO nanosheets (Fig. 9). Zhang's group [139] recently synthesized the flower-like CuS hollow microspheres based on self-assembly of nanoflakes. Many researches have demonstrated self-assembly is easy to occur in EG solution because of high viscosity. When the nanoflakes of CuS form in solution, they will be frizzled with each other to decrease the surface energy. At the same time, Deng and co-workers [140] also reported the hierarchically structured CuS hollow microspheres using template-free and green sonochemical synthesis. The authors used copper acetate monohydrate and thiourea as precursors. Hydrolysis of thiourea releases the sulfide anion in the sonication bath, which directly reacts with $\mathrm{Cu}^{2+}$ anion to form CuS.

The precipitation process is also an effective tool to fabricate hydroxide, which can be further decomposed to form oxides. Recently, Huang et al. [141] reported the shape change of cuprous oxide NCs from cubic to rhombic do- 


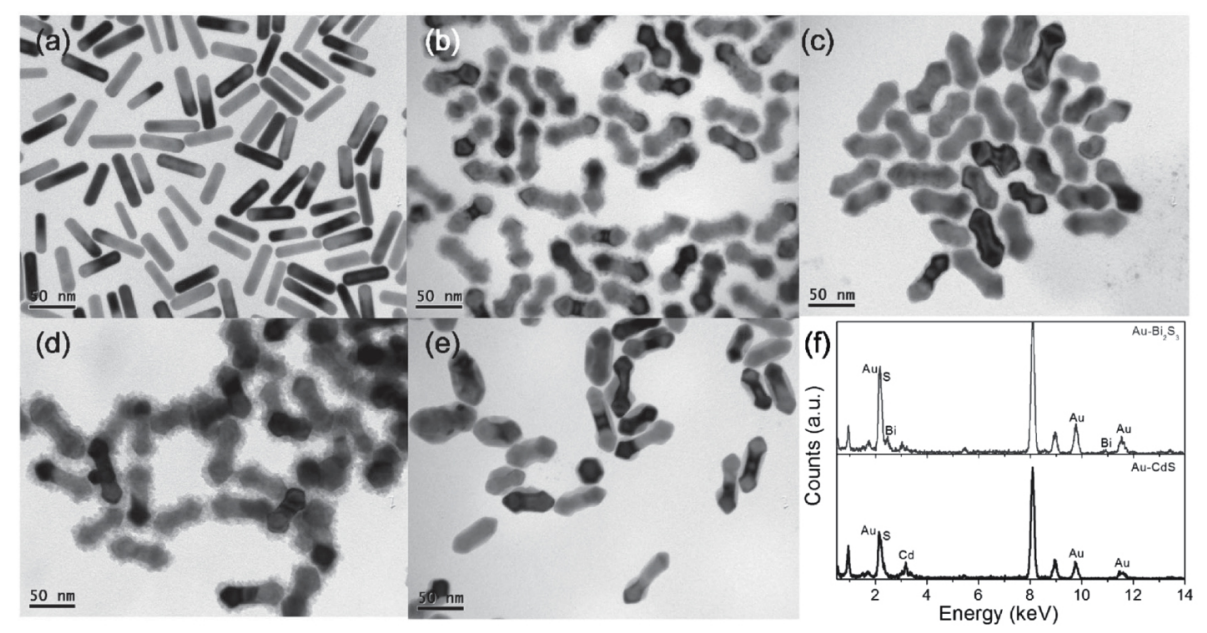

Figure 8 TEM images of initial Au nanorods (a), dumbbell-like Au-CdS (b), Au- $\mathrm{Bi}_{2} \mathrm{~S}_{3}$ (c), Au-ZnS (d), and Au-Cu $\mathrm{S}$ (e) core-shell nanorods. (f) EDS spectra of dumbbell-like Au- $\mathrm{Bi}_{2} \mathrm{~S}_{3}$ and Au-CdS core-shell nanorods on copper grid. Reproduced with permission from Ref. [137], Copyright 2015, WILEY-VCH Verlag GmbH \& Co.
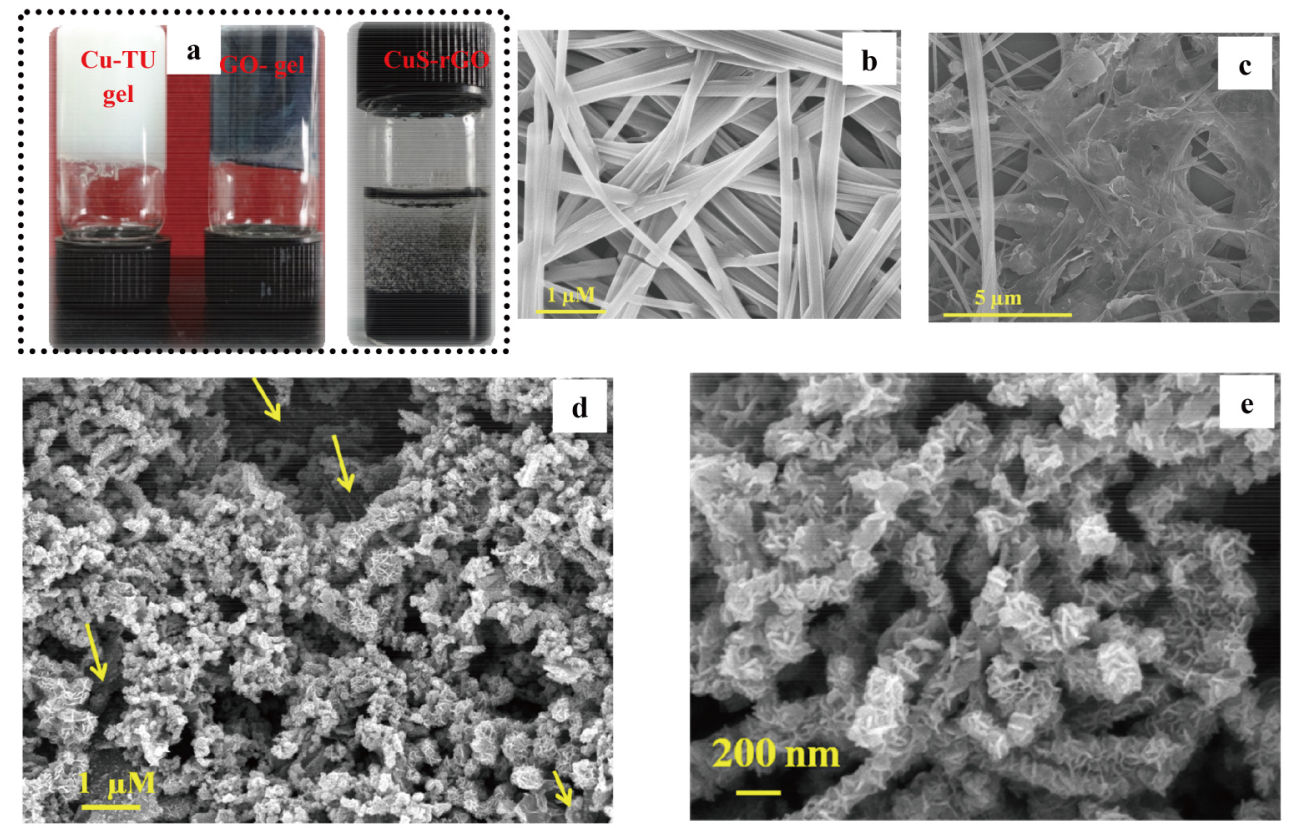

Figure 9 (a) Digital images of Cu-TU gel, GO-gel, and CuS-r-GO nanocomposites. Field emission scanning electron microscopy images of (b) Cu-TU gel, (c) GO-gel, and (d, e) CuS-r-GO nanocomposites. Yellow arrows in (d) indicate the r-GO support. Reproduced with permission from Ref. [138], Copyright 2015, American Chemical Society.

decahedral structures. These different shapes of NCs were simply achieved by adjusting the amounts of $\mathrm{NH}_{2} \mathrm{OH}-\mathrm{HCl}$ in the aqueous solution system containing $\mathrm{CuCl}_{2}$ and $\mathrm{NaOH}$. In this precipitation, intermediate product $\left(\left(\mathrm{Cu}\left(\mathrm{H}_{2} \mathrm{O}\right)_{5}(\mathrm{OH})\right)^{+}\right)$is very sensitive to $\mathrm{pH}$ which is key to ultimate geometrical morphology of $\mathrm{Cu}_{2} \mathrm{O}$. Shang et al. [142] also synthesized cuprous oxide NCs with inerratic cube and tetrahedra. They put forward a novel strategy based on recrystallization-induced self-assembly for the construction of $3 \mathrm{D} \mathrm{Cu}_{2} \mathrm{O}$ superstructures. Controlling the hydrolysis and recrystallization rates of the $\mathrm{CuCl}$ precursors through precisely adjusting the experimental parameters is key to success. In the earlier 2010, Yang's group [143] synthesized hollow $\mathrm{Cu}_{2} \mathrm{O}$ NPs through $\mathrm{Cu}$ NPs as templates at room temperature. They described the solution-phase synthesis of highly monodisperse $\mathrm{Cu}$ NPs and by controlling the oxidation process, the formation of $\mathrm{Cu} @ \mathrm{Cu}_{2} \mathrm{O}$ core/shell structures, hollow $\mathrm{Cu}_{2} \mathrm{O}$ nanospheres, 
and solid $\mathrm{Cu}_{2} \mathrm{O}$ nanospheres. Layered double hydroxides (LDHs) are a class of ionic lamellar materials owning the chemical formula of $\left[\mathrm{M}_{1-x}{ }^{2+} \mathrm{M}_{x}^{3+}(\mathrm{OH})_{2}\right]\left[\mathrm{A}^{n-}\right]_{x / n} \cdot z \mathrm{H}_{2} \mathrm{O}$ $\left(\mathrm{M}^{2+}, \mathrm{M}^{3+}\right.$, and $\mathrm{A}^{n-}$ represent di-, tri-valent metal ions and $n$-valent anions, respectively). LDHs are composed of positively charged brucite-like layers with metal cations in the center of hydroxide-occupied octahedra, intercalated anions and solvation molecules. Wu et al. [144] reported how to use the $\mathrm{Co}^{2+}\left(\mathrm{Ni}^{2+}\right)$-oleylamine (OAm) complexes as the precursor to generate $\mathrm{CoNi} \mathrm{LDH} / \mathrm{CoO}$ nanosheets via an in-situ reduction and co-precipitation process. It is noteworthy that the strong extrusion of hydrated metal-cluster resulting from interfacial tension plays a crucial role in constructing this hybrid structure. O'Hare and Wang [145] reviewed the precipitation process in the synthesis of LDHs and their corresponding applications. As a bottom-up strategy, the co-precipitation of two metal ions is the dominant method to construct a variety of LDHs. Later Xu's group [146] gave a comprehensive review about the catalytic applications of LDHs, which were strictly dependent on the synthesis conditions. Recently, Gong et al. [147] denoted a mini review of NiFe-based materials such as NiFe-LDHs as a superior catalyst for the electrocatalysis such as oxygen evolution process.

The coordination process to generate insoluble complex can be also considered as the precipitation process. This coordination is usually found in the synthesis of metal clusters, MOFs, polyoxometalates (POMs), and so on. POMs are a subset of metal oxides that can be viewed as transferable building blocks. Ever since the first report by Berzelius in 1826, POMs have drawn increasing attentions due to their diverse range of molecular structures, outstanding physical and chemical properties, and a wide range of applications [152-158]. POMs clusters are made up of metal ions $(\mathrm{M}=\mathrm{W}, \mathrm{Mo}, \mathrm{V}, \mathrm{Nb}$, etc.) and oxo ligands. In general, oxoanions are monomers in alkaline solution and assemble into polynuclear clusters in acidic conditions. In the precipitation of POMs, the countercations such as ammonium or phosphonium cations as well as the most commonlyused alkalis can play a decisive role. In general, the steric hindrance and charge density should both be concerned. For the alkali POMs, the alkali $(\mathrm{Li}, \mathrm{Na})$ with smaller size can facilitate the dissolution. By contrast, the larger ones ( $\mathrm{Rb}$, Cs) are beneficial for rapid precipitation. The larger alkalis can not only link to a single anion, but also serve as bridges between anions. Organic moieties can be added to the lacunary POMs to form a hybrid structure. Wu and co-workers [148] reported a series of surfactant-encapsulated and organically grafted POMs through a co-precipitation process
(Fig. 10a). Two different hierarchical self-assembled structures can be arranged by the complex in mixed organic solvents such as dichloromethane and methanol. Actually, the POMs can also serve as protecting ligands for decoration of NPs. Numberous examples of POM-protected nanoscale inorganic materials such as metals [159,160], oxides [161], halides [162] and graphene oxide (GO) [163] have been reported.

Over the past decades, porous solid materials are becoming one of the most promising materials due to their outstanding properties in adsorption, separation [149], purification, catalysis [164] and so on [165-167]. Among the porous solid materials, the classical examples are zeolites [168] (crystalline aluminosilicates with interconnected pores of 4-13 $\AA$ ), activated carbon [169] (usually prepared from carbonization of organic materials), and new emerging covalent organic frameworks (COFs) (linking organic building blocks by covalent bonds) and MOFs (made up with organic linkers and inorganic metal or metal-containing cluster). Fig. 10b presents the typical coordination process to generate MOFs, which is mainly discussed in this section. Because the excellent regularity, rigidity/flexibility, variety, and designability, MOFs as a new class of porous solid materials are regarded as advanced promising materials with ability of reaching or surpassing current porous materials. Clarifying the structural features and interaction between ligands and anodes are useful to achieve better synthesis of MOFs and their applications. (1) The coordination process usually takes place at mild conditions, which means that the experimental conditions can be easily controlled. (2) The organic parts of MOFs can be rationally designed based on the development of organic chemistry. (3) The coordination regularity between the organic ligands and metal ions makes the synthesis process tolerate the variation of reaction system to some extent. As a result, the research area of MOFs has undergone an eruptive development in recent years. Conceptually, the shape and/or linkage geometries of organic linkers and metal-containing nodes can be predicted. Perry et al. [170] summarized the design and synthesis of MOFs by selectively choosing the building blocks. They reviewed how the building blocks affect the shape, pore size, symmetry and stability of MOFs. For the designability, Yaghi and colleagues [171] have largely improved the development of MOFs fields and promoted the systematic synthesis of MOFs. They mentioned that the metal-containing clusters can serve as secondary building units (SBUs). The SBUs are designed and reproduced in a combination with other similar linkers. Based on this thought, the realm of MOFs 
a

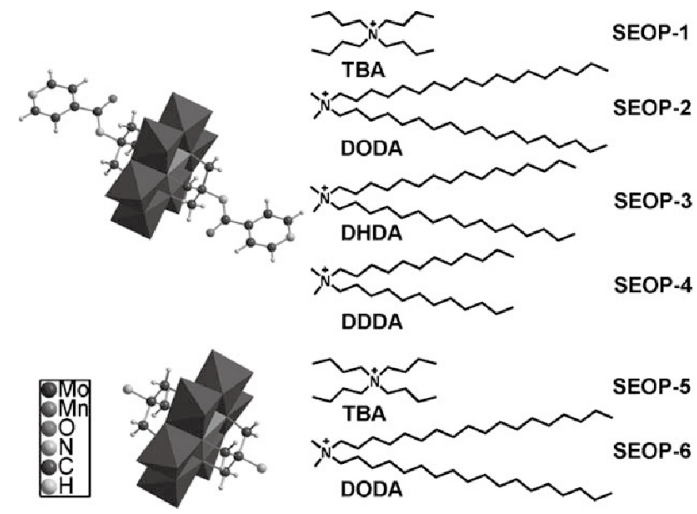

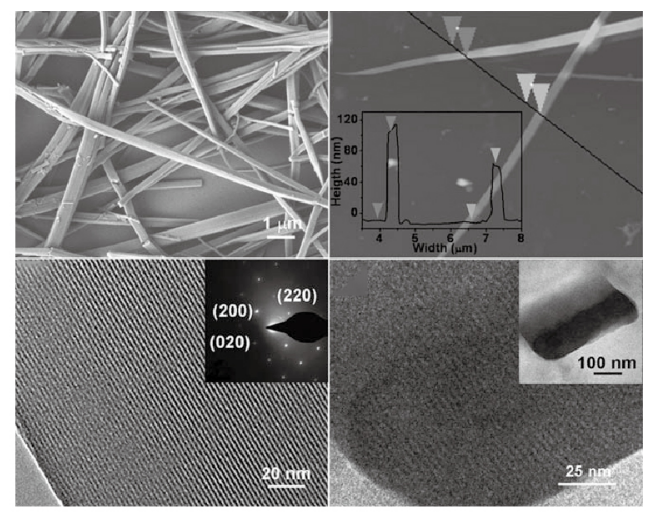

b

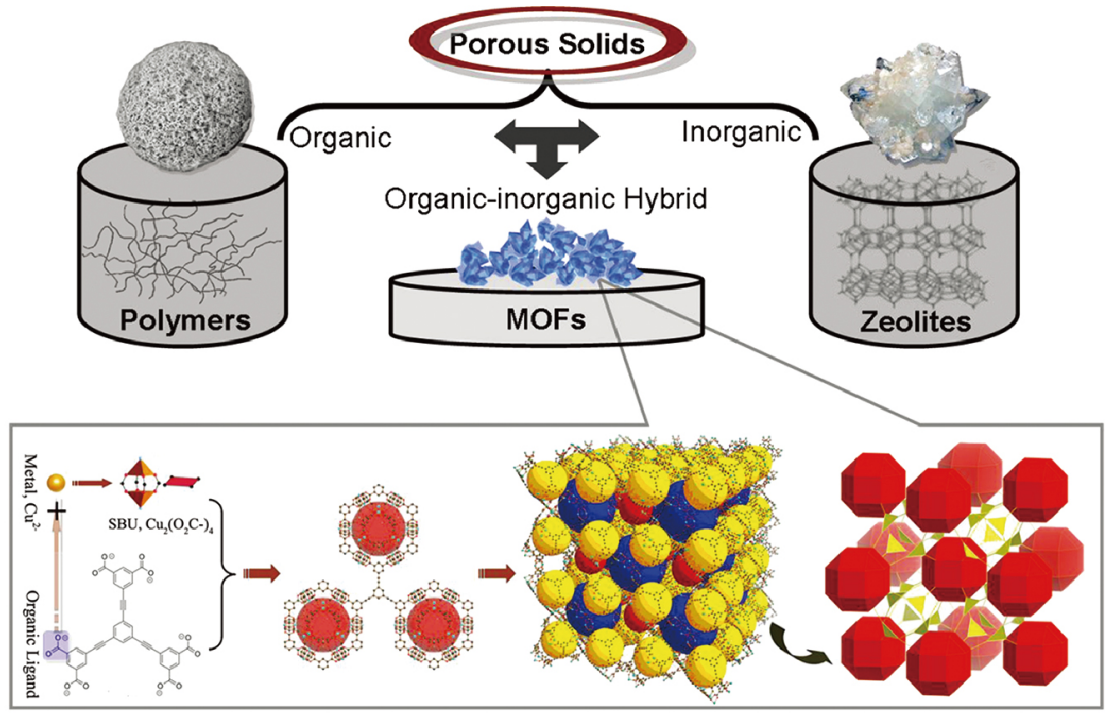

c
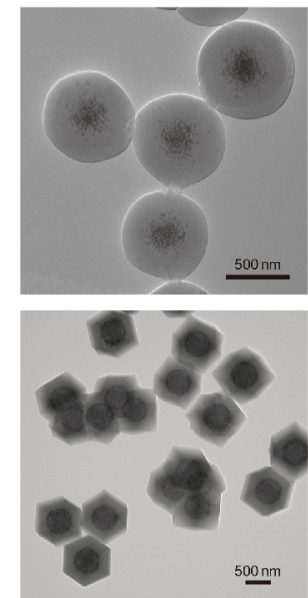
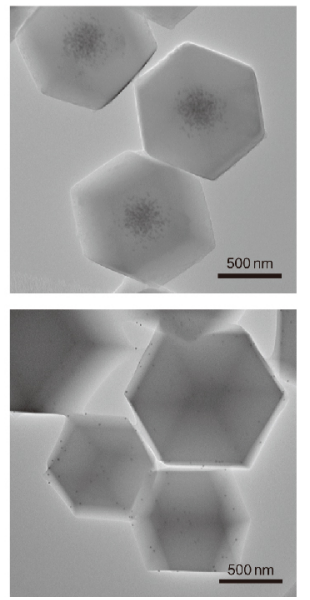

d

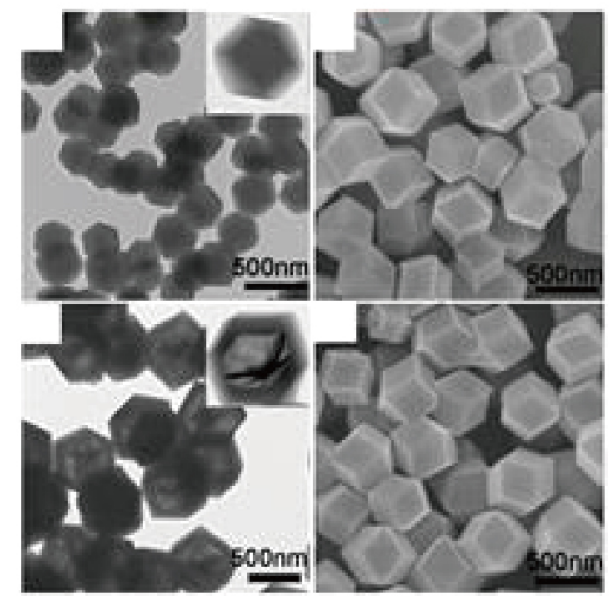

Figure 10 (a) Chemical structures of organically grafted Anderson-type POMs and surfactants employed for the preparation of surfactant-encapsulated organically grafted POMs. Reproduced with permission from Ref. [148], Copyright 2011, WILEY-VCH Verlag GmbH \& Co. (b) Schematic representations of the general classification of porous solids (top, an example is given in each case: polymers for porous organic solids; zeolites for porous inorganic solids; and MOFs for porous organic-inorganic hybrid solids) and a typical construction procedure of a MOF (bottom). Reproduced with permission from Ref. [149], Copyright 2011, American Chemical Society. (c) TEM observations of the encapsulation of $13 \mathrm{~nm}$ Au NPs in ZIF-8 crystals. Reproduced with permission from Ref. [150], Copyright 2012, Nature Publishing Group. (d) TEM images of the ZIF-67@ZIF-8 core-shell and yolk-shell structures. Reproduced with permission from Ref. [151], Copyright 2015, WILEY-VCH Verlag GmbH \& Co. 
has been greatly expanded under precisely controlled synthetic conditions. $\mathrm{Li}$ and $\mathrm{Du}$ [172] indicated that the solvent molecules may also take part into the formation of MOFs in some cases and should be carefully considered. Recently, Eddaoudi and co-workers [173] reviewed how to utilize supermolecular building approach to construct functional MOFs. This indeed assists us to rationally fabricate the desired functional MOFs. Huo's group [150] developed an encapsulation strategy which enabled several types of NCs to be incorporated fully within a readily formed ZIF-8 (Fig. 10c). This method offers an effective tool to combine the functionalities of NCs and MOFs together. Our group discovered that methanol can modify the coordination mode of ZIF-67 in the presence of $\mathrm{Co}^{2+}$ and induce a mild phase transformation under solvothermal condition [151]. By introducing the transformation process into the ZIF-67@ZIF-8 core-shell structures, a well-defined hollow Zn/Co ZIF rhombic dodecahedron can be programmed installed. The manufacturing of hollow MOFs enables us to yield the noble metal@MOF yolk-shell composite with controlled spatial distribution and morphology (Fig. 10d). In 2013, Suh and co-authors [174] highlighted various preparative strategies and characterization for metal NCs integrated with MOFs. Afterwards, Xu's group [175] contributed a detailed review about how to integrate MOFs to functional materials to form composites with special emphases on the synergistic effects.

In summary, precipitation reaction is the basic chemical reaction in the inorganic chemistry and how to control the morphology with good crystallinity is still urgently needed.

\section{Reduction reaction}

Reduction reaction is a key reaction type among the various chemical reactions with the obtaining of electron or the misregistration of electron pair between the molecules, atoms or ions. Meanwhile, as an unalienable part involved in the full reaction, there must be an oxidation reaction corresponding to the reduction ones. The diverse characteristics of the chemical reactions do us a favor to classify them as different reaction types; nevertheless, we can divide the chemistry into two types, redox reaction and non-redox reaction, by following the principle that whether there is misregistration of electron or electron pair in the reaction system, implying the significance of the redox reaction. Taking a deep insight into the redox reaction system, the species called oxidant do get a decrease of the oxidation state, oxidizing other ones and leaving itself being reduced, which is pertaining to the reduction part; on the contrary, the species called reductant do get an increase of the oxidation state, reducing the other ones and leaving itself being oxidized, which is pertaining to the oxidation part. Generally speaking, the reductant possesses a higher reducibility than the reduzate and the oxidant performs a stronger oxidability than the oxydate, according to which we can obtain the weak reductant/oxidant by the use of the strong ones.

\section{Reduction reaction for monometallic NCs}

As the foremost chemistry reaction, people have been actively applying the reduction reaction into a wide variety of fields, including the nanoscale science, electrochemistry, energy harvesting/conversion/storage, catalysis and various organic reactions [176-178]. According to statistics, almost $50 \%$ of the reactions consisted in the chemical industry come down to the redox reactions. Actually, the development of chemistry stems from the redox reaction. However, there are still some challenges to deeply understand the mechanism of the redox reaction, including how the misregistration of electron happens, the control of the reaction rate and so on. Table 1 summarizes some of the monometallic and bimetallic NCs with well-defined shapes by reduction.

In the past several decades, interest in nanoscale science and technology has been grown sequentially owing to their special role as bridge combining atoms and bulk solids. The NCs, noble or non-noble metal [180], oxide [181], semiconductor [182] and so on, show fascinating properties and potential applications. Above all, the noble or non-noble metallic NPs deserve our special attention due to their widespread applications ranging from catalysis, photography, information storage to imaging and medicine [183-185]. Generally speaking, whatever the metallic NCs that we get are pure metal or alloy, there are two steps, nucleation and growth, consisted in the formation of a metallic NC. In a typical synthesis of metal NCs, a precursor can generate zero-valent atoms to build blocks of a metal NC by either decomposition or reduction. Yet, how nuclei and NCs evolve from a precursor is still unclear. For the reduction rate, oxidation state of the precursor compound is higher than the atomic species. In this case, there are two ways to form metal NCs from a precursor compound. For the first possibility, the precursor is reduced into zero-valent atoms, which would sequentially aggregate into nuclei and finally grow into NCs. For the second possibility, the unreduced precursor compound begins forming nuclei prior to reduction. Without going through a zero-valent state, the precursor compounds can be directly convered into dimers and trimers. Reduction 
Table 1 Summary of different shapes that have been achieved for various metal NCs by reduction

\begin{tabular}{|c|c|c|}
\hline $\begin{array}{l}\text { Monometallic } \\
\text { nanostructure }\end{array}$ & Shapes & Metals \\
\hline \multirow[t]{6}{*}{ Single-crystals } & Perfect/truncated cube & $\begin{array}{c}\text { Pd [208,240], Ag }[43,64,241], \mathrm{Au}[242,243], \mathrm{Pt}[244,245], \mathrm{Cu}[246] \\
\text { Rh [247], Fe [248] }\end{array}$ \\
\hline & Concave cube & $\operatorname{Pd}[219]$ \\
\hline & Perfect/truncated octahedron & Pd $[193,194], \operatorname{Ag}[43,64], \mathrm{Au}[242,243], \mathrm{Pt}[244,245]$ \\
\hline & Perfect/truncated tetrahedron & $\operatorname{Ag}[241], \mathrm{Au}[243,249], \mathrm{Pt}[45,244]$ \\
\hline & Rectangular bar & $\operatorname{Pd}[186]$ \\
\hline & Multipods & $\mathrm{Au}[250], \mathrm{Pt}[244,245,251], \mathrm{Rh}[247]$ \\
\hline Singly twinned & Right bipyramid & Pd [252], Ag [253] \\
\hline \multirow[t]{4}{*}{ Multiply twinned } & Decahedron & $\mathrm{Pd}[193], \mathrm{Ag}[254], \mathrm{Au}[243,249]$ \\
\hline & Icosahedrons & Pd [193], Au [249] \\
\hline & Five-fold twinned pentagonal rod/wire & $\mathrm{Pd}[255], \mathrm{Ag}[256], \mathrm{Au}$ [257], $\mathrm{Cu}[255]$ \\
\hline & Triangular/hexagonal plate & $\begin{array}{c}\mathrm{Pd}[258], \mathrm{Ag}[259,260], \mathrm{Au}[261], \mathrm{Cu}[246], \mathrm{Pb}[262], \mathrm{Co}[263] \\
\mathrm{Ni}[264]\end{array}$ \\
\hline \multicolumn{3}{|c|}{ Bimetallic nanostructure } \\
\hline \multirow[t]{9}{*}{ Alloy } & Cube & Pt-M [230,232,265-268] \\
\hline & Tetrahedron/octahedron & Pt-M [230,265-267] \\
\hline & Icosahedron & Pt-Pd [269] \\
\hline & Rod & $\mathrm{Pt}-\mathrm{Fe}[270], \mathrm{Pt}-\mathrm{Cu}[271]$ \\
\hline & Multi-pod & $\mathrm{Au}-\mathrm{Pt}[272], \mathrm{Pt}-\mathrm{Fe}[273]$ \\
\hline & Dendritic structure & Pt-Pd [274], Au-Pd [275], Pt-Co [180] \\
\hline & Wires & $\mathrm{Au}-\mathrm{Ag}[276], \mathrm{Pt}-\mathrm{Ag}[277]$ \\
\hline & Hollow structure & Pt-Pd $[78,278]$ \\
\hline & Concave structure & Au-Pd [105], Pt-Cu [279] \\
\hline \multirow[t]{4}{*}{ Core-shell } & Cube & $\begin{array}{c}\text { Pt@Pd [231,280], Au@Pd }[220,225,281,282], A u @ A g[225], \\
\text { Cu@Pd [283] }\end{array}$ \\
\hline & Octahedron & Pt@Pd [284], Pd@Pt [285] \\
\hline & Core-dendritic shell & Pd@Pt [286], Pd@Rh [287], Au@Pd@Pt [288] \\
\hline & Tetrahexahedron/tris-octahedron & Au@Pd $[289,290]$ \\
\hline \multirow[t]{3}{*}{ Hybrid structure } & Rod & $\mathrm{Au}-\mathrm{Ag}[291]$ \\
\hline & Binary structure & $\mathrm{Au}-\mathrm{Pt}[231], \mathrm{Pt}-\mathrm{Pd}[229]$ \\
\hline & Multi-pod & Pd-Pt [292] \\
\hline
\end{tabular}

occurs by the electron transfer from the reductant to dimers and trimers, because the electron affinities of dimers and trimers are higher than that of the precursor. It is worth pointing out that only under certain experimental conditions, such as an appropriate reducing agent and a high concentration of precursor, the reduction mechanism occurs. For example, Ciacchi et al. [179] utilized the first principles molecular dynamics to investigate the growth of Pt clusters by the addition of $\mathrm{Pt}$ (II) complexs to the cluster, which may be the initial process of nucleation (Fig. 11). In their simulations, there are no electrons transfer during the reaction between $\mathrm{PtCl}_{2}\left(\mathrm{H}_{2} \mathrm{O}\right)_{2}$ complexes and $\mathrm{Pt}_{12}, \mathrm{Pt}_{12} \mathrm{Cl}_{4}$, or $\mathrm{Pt}_{13} \mathrm{Cl}_{6}$ clusters. According to the evaluation of electronic density of states, the growing clusters extend its electronic states over the whole cluster after the adsorption of the $\mathrm{Pt}(\mathrm{II})$ complex. Two dissolved $\left[\mathrm{PtCl}_{2}\left(\mathrm{H}_{2} \mathrm{O}\right)_{2}\right]$ complexes can directly form $\mathrm{Pt}(\mathrm{II})-\mathrm{Pt}(\mathrm{I})$ dimer which is stabilized with $\mathrm{Cl}^{-}$ through the introduction of one electron. After the introduction of an electron and the loss of $\mathrm{Cl}^{-}$, the $\mathrm{Pt}(\mathrm{I})-\mathrm{Pt}(\mathrm{II})$ dimer can be transformed subsequently into a $\mathrm{Pt}(\mathrm{I})-\mathrm{Pt}(\mathrm{I})$ dimer. Interestingly, a trimer can be formed from the reaction of $\left[\mathrm{PtCl}_{2}\left(\mathrm{H}_{2} \mathrm{O}\right)_{2}\right]$ complex with both the $\mathrm{Pt}(\mathrm{II})-\mathrm{Pt}(\mathrm{I})$ and $\mathrm{Pt}(\mathrm{I})-\mathrm{Pt}(\mathrm{I})$ dimers after a reduction process. We positively believe these partially reduced dimers and trimers 

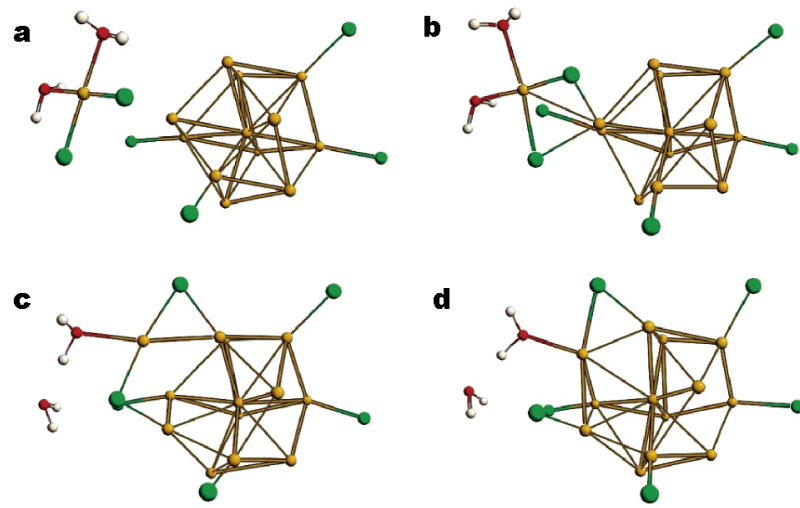

d

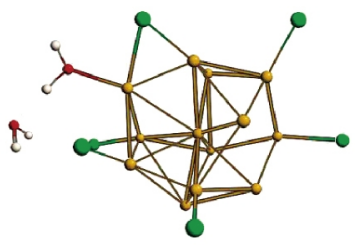

e

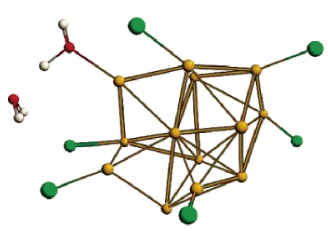

in a water-based system, achieving the control of the reduction kinetics, and thus the morphology of Pd NCs (Fig. 13) [191]. We were able to achieve various shapes of metal NCs by controlling the reduction rate and concentration of the precursor. For example, with only $\mathrm{Na}_{2} \mathrm{PdCl}_{4}, \mathrm{PVP}$, and citric acid added in a simple water-based system, many shapes of Pd NCs can be achieved, such as nanowires [80], nanocubes [192], decahedra [193], icosahedra [79], octahedra [194], five-fold twinned nanorods, single-twinned right bipyramids [195], triangular nanoplates and so on. The reduction rate and the concentration of precursor are the two main factors to affect the atomic concentration. The concentration of the Pd precursor has to be kept relatively high in such process, since citric acid is a mild reducing agent.

AA possesses a strong reducing capacity for fast reduction of Pd precursors, which is crucial to the formation of thermodynamically favorable Pd truncated octahedra. Nevertheless, citric acid serves as a moderate reducing agent as well as a surface capping agent, observing a similar mechanism of the traditional citrate-based synthesis. The citric acid prefers to strongly bind to the facets of $\mathrm{Pd}$, blocking oxidative etching, to selectively synthesis Pd icosahedrons and decahedrons by adjusting the concentrations of citric acid and Pd precursor. Notably, PVP was employed in a manner similar to a long-chain alcohol with a weak reducing power, not merely being served as a stabilizer to block the agglomeration of metallic NCs. The considerablely slow reduction of Pd precursor contributed to converting the thermodynamically controlled pathway into kinetically controlled process, giving the formation of hexagonal and triangular nanoplates. Notably, the polar solvents could also play the part of reductant in some cases such as polyol synthesis, being performed for the synthesis of many metallic NPs, especially platinum group metals. The polyols possess the ability to efficiently dissolve many metal precursors and a temperature dependent reducing power range from 140 to $160^{\circ} \mathrm{C}$ [196-198]. Among the reaction temperature sections, the polyol such as EG primarily being oxidized into glycolaldehyde by the oxygen in air, rather than acetaldehyde, which has been firmly regarded as the reducing agent for the reduction of metal precursors in a typical polyol synthesis [199]. However, the polyols tend to give a fast reduction and growth rates due to the strong reducing power, limiting the shape of the noble metal NCs [198,200].

In the contrary, some weak polar organic solvents such as oleylamine, oleic acid, benzene and benzyl alcohol, are employed in organic-phase reductions, which utilize organic 
a

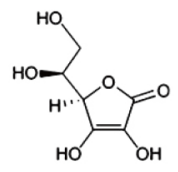

L-ascorbic acid
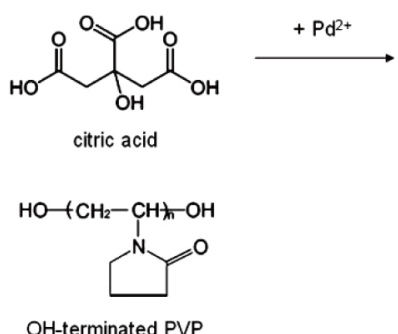

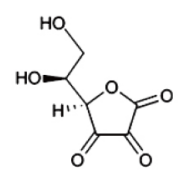

L-dehydroascorbic acid

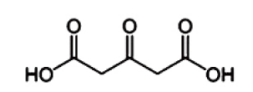

acetone-1,3-dicarboxylic acid

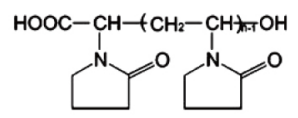

cOOH-terminated PVP b $\quad(2+)$

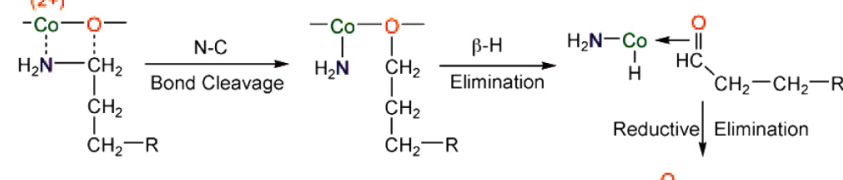

$\mathrm{R}=\left(\mathrm{CH}_{2}\right)_{5} \mathrm{CH}=\mathrm{CH}\left(\mathrm{CH}_{2}\right)_{7} \mathrm{CH}_{3}$

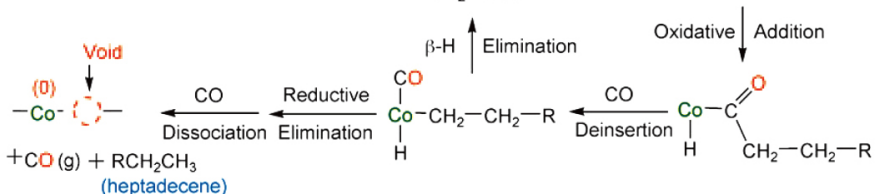

(heptadecene)

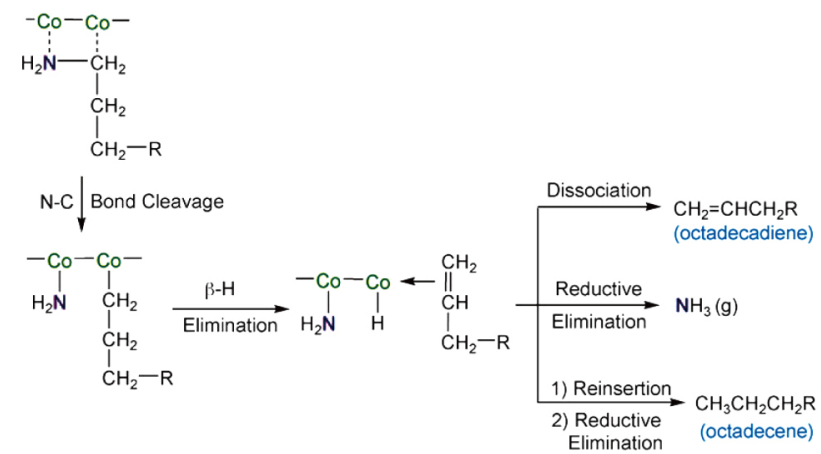

Figure 12 Structural illustration of AA, citric acid, and OH-terminated PVP and their oxidized forms due to the redox reactions with Pd ${ }^{2+}$ ions. Reproduced with permission from Ref. [186], Copyright 2009, Wiley-VCH Verlag GmbH \& Co. (b) Reduction of fcc-CoO to fcc-Co by oleylamine with formation of heptadecene and heptadecadiene (up); conversion of oleylamine into octadecene and octadecadiene by fcc Co (down). Reproduced with permission from Ref. [187], Copyright 2008, Wiley-VCH Verlag GmbH \& Co.

soluble reducing agents such as hexadecanediol, tetra-butylammonium bromide, bromothymol blue, to synthesize various metal NCs. Certainly, the weak polar organic solvents can also serve as reducing agents when the temperature exactly triggers the reduction activity of them. Beyond that, since some of these species are inclined to act as a surfactant to block the aggregation of the metal NPs, there are many advantages of organic-phase synthetic methods, favoring the preparation of high crystalline and monodispersed metallic NPs with excellent dispersion ability in organic solvents. OAm is a long-chain primary alkylamine, and it can act as electron donor at elevated temperatures. Nam et al. [187] reported the reduction of fcc-CoO to fcc-Co by OAm at $290^{\circ} \mathrm{C}$ (Fig. 12b). The combination of $\mathrm{OAm} / \mathrm{OAc}$, serving as cosurfactant, does favor for producing facetd Pt NCs in a 3-7 nm size range [201]. Controlling the reaction rates by the reaction temperature in a simple synthesis route, just heating $\mathrm{Pt}(\mathrm{acac})_{2}$ in $\mathrm{OAm}$, contributed to obtaining spherical or branched Pt NPs [202]. In addition, OAm served as a coreductant together with BTB to produce $4-5 \mathrm{~nm}$ Pd NPs which played a part of a solvent and surfactant at the same time [203]. With respect to the $\mathrm{Pd}(\mathrm{OAc})_{2}$ concentration, the ratio of
OAm/OAc is necessary to be excess for the synthesis of $\mathrm{Pd}$ NPs with $3.0 \mathrm{~nm}$ by the reducing power of tetra-butylammonium bromide (TBAB) [204]. Furthermore, OAm tends to form the $\left[\mathrm{Pd}(\mathrm{acac})_{x}(\mathrm{OAm})_{y}\right]$ intermediate complexes when there is formaldehyde in the reaction, making for a great rate control of the synthesis, then a shape control over the final NPs [205]. Niu et al. [206] discovered the oleylamine-mediated shape evolution of Pd NCs and obtained the icosa-, deca-, octa-, tetrahedral, and triangular platelike Pd NCs (Fig. 14). We reported that this synthesis followed a three step "intermediates formation-nucleation-growth" process, during which OAm played a key role in the shape evolution of Pd NCs by mediating the counterbalance between crystal strain and surface energy.

Besides the widely application of soluble solid or miscible liquid reductant for the synthesis of NCs, gas reducing agents, such as $\mathrm{CO}$ or $\mathrm{H}_{2}$, have been introduced to synthesize metal or alloy NPs. CO plays a crucial role in controlling the shape over the final NPs as well as favors the nucleus and seed formation. CO prefers to adsorb on the $\{100\}$ facets of Pt NPs, effectively stabilizing the $\{100\}$ facets, and thus benefits the formation of both cubic Pt and Pt alloy NPs. DeSantis and Skrabalak [207] obtained Pt nanocubes 

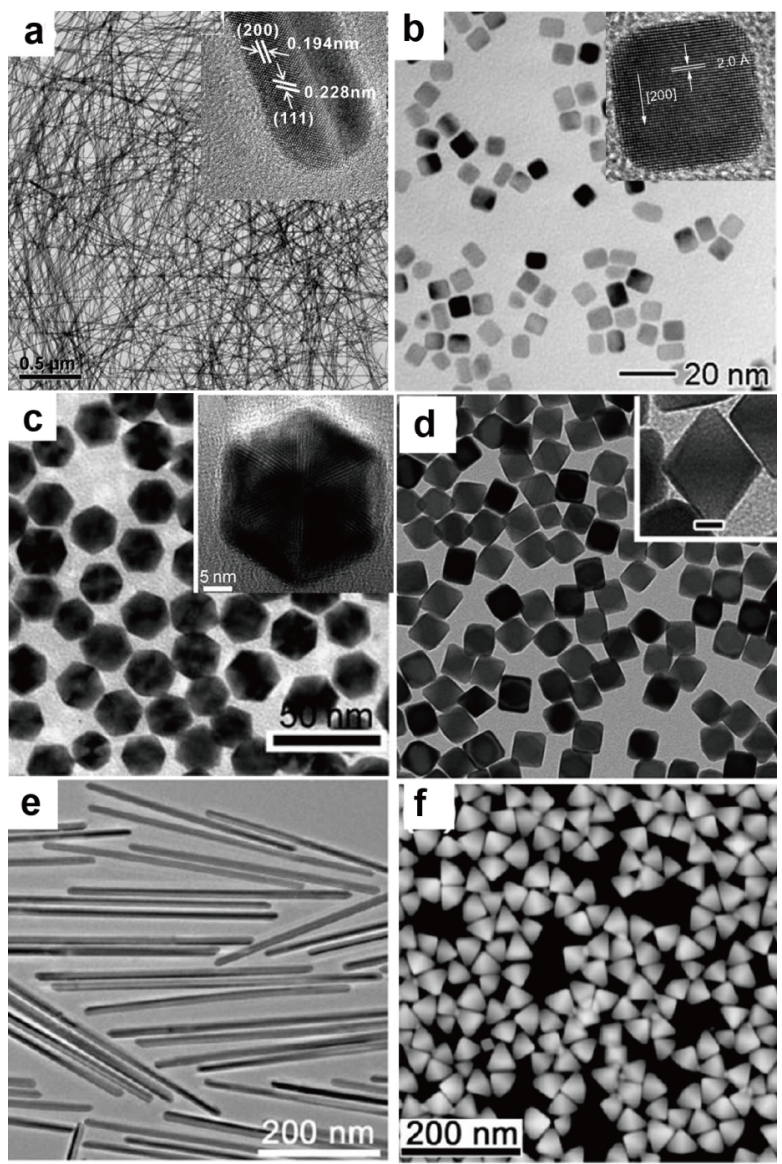

Figure 13 Shape control synthesis of Pd (a) nanowires (reproduced with permission from Ref. [80], Copyright 2009, American Chemical Society); (b) nanocubes (reproduced with permission from Ref. [192], Copyright 2005, American Chemical Society); (c) icosahedra (reproduced with permission from Ref. [79], Copyright 2009, WILEY-VCH Verlag GmbH \& Co.); (d) octahedra (reproduced with permission from Ref. [194], Copyright 2012, Royal Society of Chemistry); (e) nanorods and (f) bipyramids (reproduced with permission from Ref. [195], Copyright 2012, American Chemical Society).

by using $\mathrm{CO}$ as a reductant in OAm. However, some other morphology such as nanowires, nanoplates, and octahedra can also be synthesized by combining $\mathrm{CO}$ gas with different capping agents. Similar to $\mathrm{CO}$, the $\mathrm{CO}$-containing organometals such as $\mathrm{Fe}(\mathrm{CO})_{5}, \mathrm{~W}(\mathrm{CO})_{6}$, and $\mathrm{Co}_{2}(\mathrm{CO})_{8}$ likewise possess the similar ability to tune the morphology of the final NPs, due to the CO decomposed from the carbonyls along with the reaction process. The $\mathrm{Pt}$ nanocubes in a $8 \mathrm{~nm}$ size obtained by introducing trace amount of $\mathrm{Fe}(\mathrm{CO})_{5}$ in octadecene, with the combination of $\mathrm{OAm} / \mathrm{OAc}$, were consistently reported by several research groups [208-210]. Afterwards, $\mathrm{Hu}$ and co-authors [211] presented that the $\mathrm{W}(\mathrm{CO})_{6}$ can be used to generate the Pt-It nanocubes bounded with $\{100\}$ facets. Absolutely,
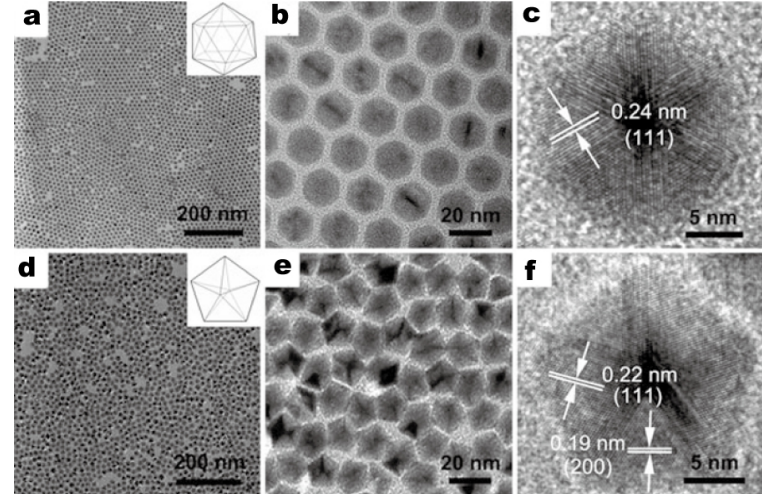

g
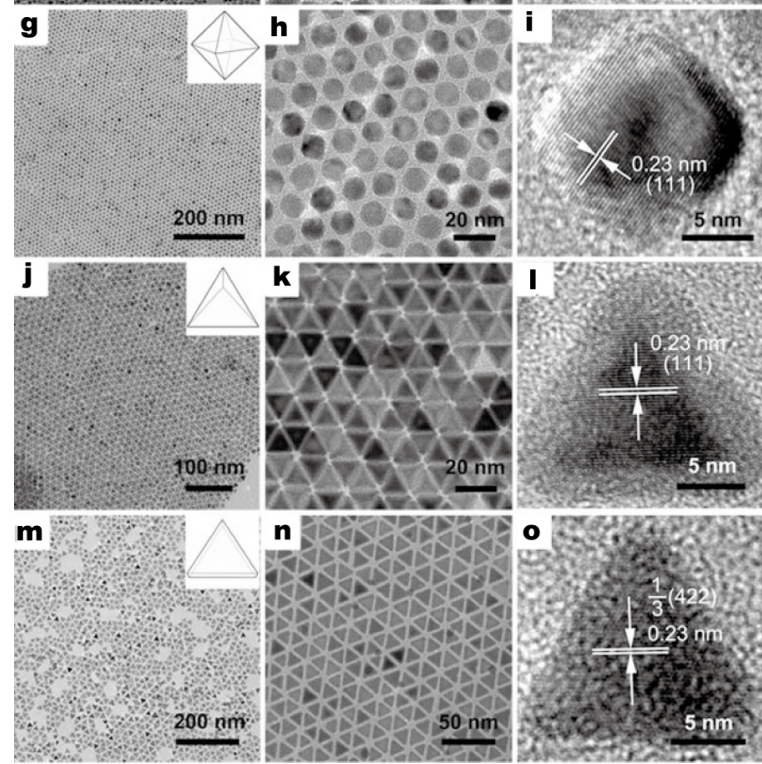

Figure 14 TEM images of Pd NCs with different shapes: $(a-c)$ icosahedron, (d-f) decahedron, $(\mathrm{g}-\mathrm{i})$ octahedron, $(\mathrm{j}-\mathrm{l})$ tetrahedron, and $(\mathrm{m}-\mathrm{o})$ triangular plate. Reproduced with permission from Ref. [206], Copyright 2009, WILEY-VCH Verlag GmbH \& Co.

further endeavors should be made to better understand the kinetic effects of $\mathrm{CO}$ gas so as to achieve an efficiently utilization of $\mathrm{CO}$ gas in the synthesis of colloidal metal and metal alloy NCs. Actually, to generate the $\mathrm{CO}$ as the shape dictating agents, $\mathrm{Xu}$ et al. [212] reported the small adsorbates of $\mathrm{CO}$ can be obtained from the formamide, which also served as a solvent, to assist the shape control of NCs. They utilized this novel synthetic system to regulate a set of well-defined nanostructures such as $\mathrm{Pd}-\mathrm{Fe}_{2} \mathrm{O}_{3}$. Apart from capping agent, $\mathrm{CO}$ can also serve as reducing agent in liquid solution to facilitate the reduction process. Recently, $\mathrm{Wu}$ et al. [213] synthesized stable sub-two nanometer AgAu nanowires by using $\mathrm{CO}$ as the gas reducing agent in liquid solution (GRAILS) method. The CO can be used both as the capping agent and reductant, which is crucial to realizing the shape control over AgAu nanowires bounded by 
$\{111\}$ facets.

Wang's group [214] contributed a detailed review about controllable synthesis of noble metal ( $\mathrm{Au}, \mathrm{Ag}, \mathrm{Pd}, \mathrm{Pt}$ ) nanomaterials and application in fuel cells and analytical sensors. Xu's group [215] contributed a detailed review about synthesis and application of noble metal NCs with high-energy facets. In this review, by kinetic controlling the growth of noble metal NCs especially for $\{100\}$ facets and high-index facets, a series of versatile approaches to synthesize NCs with thermodynamically unstable facets [216-218] were clearly discussed. By controlling the growth rate of metal NCs along the different directions, high-energy facets could be formed [6]. For example, Xia's group [219] synthesized concave cubic Pd NCs enclosed by $\{730\}$ high-energy facets through a kinetically controlled process. The depositions of metal atoms on the corner and edges of the cubic seeds can be promoted with lower diffusion rate of the precursor and faster reduction rates [220]. Thus, with a higher concentration of AA and a lower concentration of $\mathrm{H}_{2} \mathrm{PdCl}_{4}$, the $\mathrm{Pd}$ atoms deposited on the corner and edges of the cubic Pd seeds, leading to the formation of concave cubic Pd NCs enclosed by $\{730\}$ high-energy facets. In 2009, a classical review about the nucleation and growth of shape controlled synthesis of noble metal NCs was summarized by Xia et al. [2]. In this review, the chemistry and physical factors involving in the synthesis of metal NCs such as twin, defect, oxidative etching, capping agents, redox potential, epitaxy, and so on were overviewed. Later, Sun [221] contributed a similar review focusing on the synthesis of colloidal Ag NCs in organic solutions. Further, the controlled preparation of metal NCs with highly branched morphologies [3] and concave structures [4] were reviewed by Xia's group, respectively. Naik's group [222] reviewed how to use biomolecules to achieve the specific controlled synthesis of metal nanomaterials. Most recently, Du and co-workers [223] contributed a mini-review about the freestanding ultrathin metallic nanosheet. It is worth noting that constructing the fcc metals into the $2 \mathrm{D}$ nanosheets is still a great challenge due to its low anisotropy of unit cell.

\section{Reduction reaction for bimetallic NCs}

Compared with monometallic NCs, bimetallic NCs usually exhibit better catalytic activity by dictating the charge transfer between two different metals, the lattice strain introduced by the alloying process, surface coordination environment and elemental segregation. Hence, the reduction potential of specific metals, relative decomposition/reduction rate of metal precursors and interfacial energy be- tween two metals are believed to play a crucial role in modulating the shape of bimetallic NCs. It is believed that five factors can control the shape of bimetallic NCs. The first factor is the redox potentials of different metals. In reducing a metal precursor, standard reduction potential (SPR) is always utilized to evaluate the difficulty of reduction of metal from ionic precursor. The easier the metal precursor can be reduced, the more positive SPR is. In non-aqueous solution, SPR also reveals a trend of reducibility among different metal precursors, although it is defined in aqueous solution. Since the ligands can usually strongly bind to the metal cations, we can selectively use the ligands to lower the SPR of the metal. If the reduction rate of the metal precursor significantly decreases, the reaction process will be determined by the thermodynamic factors and become near equilibrium conditions. In general, the SPRs of non-noble metals such as $\mathrm{Fe}, \mathrm{Co}, \mathrm{Ni}$ are much lower than those of noble metals such as $\mathrm{Au}, \mathrm{Pd}$ and Pt. In the typical synthesis of bimetallic NCs comprising one non-noble metal and one noble metal, we should decrease the difference of redox potential between the two metal precursors to guarantee the co-reduction of two metals. For instance, the $\mathrm{Br}^{-}$ ions can serve as the oxidation facilitator of Pd to promote the galvanic replacement reaction between $\mathrm{Pt}^{2+}$ ions and $\mathrm{Pd}$ seeds. Without $\mathrm{Br}^{-}$ions, the reaction will follow the seeded growth route during which the Pt ions are reduced by the weak reductants and the core-shell Pd@Pt NCs will form [224].

The second factor that should be considered is the interfacial energy between the two metals, which can determine the thermodynamic stability of bimetallic interface. Low interfacial energy tends to induce the layered growth of the overlayer metal. In contrast, high interfacial energy usually results in the island growth mode [225,226]. The lattice match between the overlayer and substrate metals is the main factor determining the interfacial energy [225] (Fig. 15). To confine the nucleation and overgrowth of overlayer on the substrate, another important factor is the surface diffusion of overlayer metal on the substrate. Sneed et al. [82] demonstrated an iodide-mediated epitaxial overgrowth of $\mathrm{Rh}$, during which the $\mathrm{Rh}$ islands can be epitaxially grown on the $\mathrm{Au}$ and $\mathrm{Pd}$ substrates no matter the $\{111\}$ or $\{100\}$ facets are exposed. Xia and co-workers [227] reported the addition of $\mathrm{Br}^{-}$and $\mathrm{I}^{-}$would help to control the diffusion rate of $\mathrm{Rh}$ atoms over the surface of $\mathrm{Pd}$ seeds. Combining the control over the nucleation rate of $\mathrm{Rh}$ atoms from precursor, island growth and layrered growth modes for the deposition of Rh atoms on $\mathrm{Pd}\{111\}$ facets can be selectively controlled. Afterwards, they have extended this regulation 


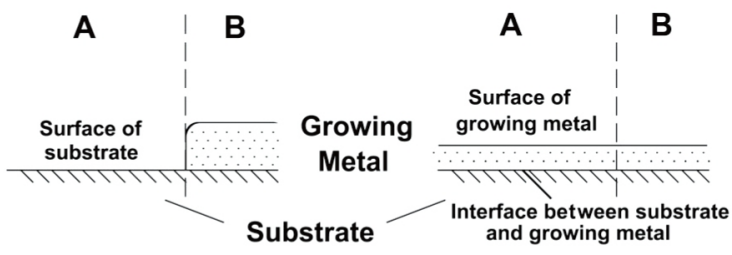

Island growth

Figure 15 Schemes of island growth model and layered growth model. Reproduced with permission from Ref. [85], Copyright 2012, Royal Society of Chemistry.

strategy to the synthesis of $\operatorname{Ir}\{100\}$ and $\{111\}$ facets covering the Pd cubes and octahedra [228].

The third factor is the reduction rate. There are many factors affecting the reduction rate of metal precursors, including the reducing capacity of the reductant, the redox potential of the precursors, the reaction temperature, etc. The shape of metallic NCs is difficult to control under fast reduction. Same to the synthesis of monometallic NCs, many reagents can be used as proper reductant, such as AA, PVP and polyol. By changing the concentration of reductant, the reduction rate can be controlled. In the nucleation stage, multi-twinned seeds can form under a low reduction rate. If the reduction rate is drastic, the consumption of metal precursors is increased and the nucleation rate is speed up, which can be considered as explosive nucleation. In the seeded growth route, multi-site growth on random surface of the given seeds can be initiated under a high reduction rate. By adjusting the $\mathrm{pH}$ value of the aqueous solution, Yang and co-workers [229] reported the reducing capacity of AA could be tuned to control the reduction of $\mathrm{K}_{2} \mathrm{PdCl}_{4}$ by AA in the presence of Pt nanocubes. In a neutral condition, the core-shell Pt@Pd structure would form since the nucleation of Pt took place on the surface of $\mathrm{Pd}$ core. When reducing capacity of AA was increased, the reduction rate of $\mathrm{PdCl}_{4}{ }^{2-}$ would significantly enhance and the localized growth of Pd islands on cubic Pt seeds could be obtained.

The fourth factor is the facet-specific capping agent. Many agents can be used as capping agents, such as salts, surfactants, gas molecules and polymers. As is well known, halide ions are one commonly used capping agents for the selective binding of the $\{100\}$ facet for most of fcc bimetallic NPs $[78,231]$. In an organic solvent system, the organic amines and acids are commonly known as typical capping agents to control the morphology of hydrophobic NCs, although the intrinsic mechanism that how these facet-specific capping agents binds to the metal surface

is still not clear. Wu et al. [230] developed a general method for the shape controlled synthesis of a series of water-soluble $\mathrm{PtNi}_{2} \mathrm{NCs}$, which utilizes PVP as the capping agent. It is shown that benzoic acid can be used as the $\{111\}$ facet-specific capping agent for the synthesis of Pt-Ni alloy (Fig. 16). Sometimes, small adsorbates and long-chain ligands can serve as co-capping agents, where $\mathrm{CO}$ is generated from the decomposition of metal carbonyl or introduced additionally [232,233].

The fifth factor is the temperature and reaction time. The decomposition and reduction rate of metal precursors will obviously increase with elevated temperature. So, it is not difficult to understand that high temperature is beneficial for the cases that reducibility of the reductant is weak and the metal precursor is hard to be reduced.

More importantly, by changing the reaction temperature the nucleation and growth regime can be further controlled. At high temperature, the growth process is mainly determined by the thermodynamical factors. That is, the growth direction tends to be isotropic and the most stable crystal facets such as low-index facets and convex polyhedra will be dominant in the resulting products. In the contrary, the growth and coalescence stage will be under kinetic control at low temperature, during which some facets owning faster growing rate are more likely to preserve. The match of the decomposition and reduction rates of the precursors can be determined by the reaction time. In general, it is easier to obtain thermodynamically favored products after long reaction time. For example, some unconventional shapes such as nanowires [234], nanoplates [235], concave NPs and NPs exposing high-index facets [45] have been reported by the controlling over reaction temperature and time.

In particular, the reaction temperature can also serve as a driving force to induce the elemental segregation in bimetallic system due to the different thermostability and mobility of two metals. In 2011, Bao's group reported that the utilization of oxygene and hydrogen can achieve the control of segregation of $\mathrm{Pt}$ and $\mathrm{Ni}$ in $\mathrm{Pt}-\mathrm{Ni}$ alloy by the distinct binding energy. They found the surface $\mathrm{NiO}_{x}$ islands offer active sites for the activation of $\mathrm{O}_{2}$, which favors the oxidation of $\mathrm{CO}$. Upon treating the bimetallic surface under oxidative or reductive atmosphere, the segregation of $\mathrm{Ni}$ and $\mathrm{Pt}$ and the oxidation of Pt can be selectively controlled [236]. Wang et al. [237] reported the sequential acid leaching and high temperature annealling could result in the multilayered Pt-skin surface in Pt-Ni bimetallic NPs. Moreover, they discovered this novel structure with a Pt-rich segregation on the surface can largely improve the 


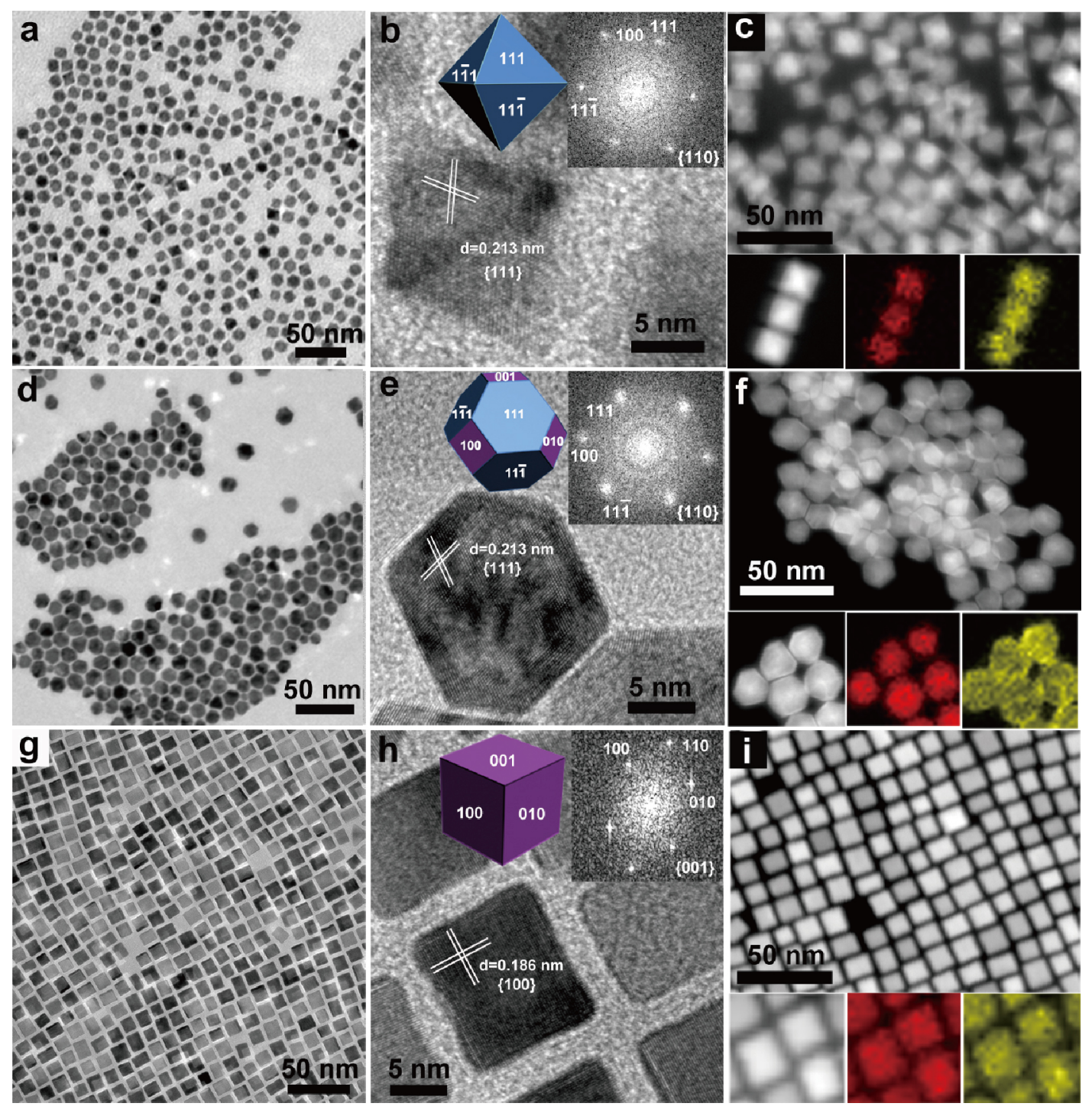

Figure 16 TEM images of $\mathrm{PtNi}_{2}$ (a) octahedra, (d) truncated octahedra and (g) cubes. HRTEM images of $\mathrm{PtNi}_{2}$ (b) octahedra, (e) truncated octahedra and (h) cubes. Top-right and top-middle insets show the corresponding FFT pattern and the ideal structure model of NC. High angle annular dark field-scanning transmission electron microscopy (HAADF-STEM) images and corresponding element maps showing the distribution of Pt (yellow) and $\mathrm{Ni}$ (red) of $\mathrm{PtNi}_{2}$ (c) octahedra, (f) truncated octahedra and (i) cubes. Reproduced with permission from Ref. [230], Copyright 2012, American Chemical Society.

electrocatalytic performance for the oxygen reduction reaction. Most recently, they gradually extended this strategy to 3D Pt-Ni frameworks by ligands-assisted oxidative etching followed by a high temperature treatment [238]. The surface segregation is a common phenomenon in bimetallic system and strictly related to the crystal structure and surface energy. Recently, Yu et al. [239] reviewed how the fine structure such as kink, defect, segregation and so on play during the catalytic cycle. They also talked about how to construct a well-defined supported Pt-based surface based on the model surface and simulations.
When the NCs are downsized to small scale (ca. $<5 \mathrm{~nm}$ ), the surface stress becomes significant and can induce structural change. In this regard, Wu et al. [293] reviewed how the surface lattice strain affects the synthesis of bimetallic NCs and their catalytic properties. Wang and co-workers [294] summarized the recent advances in constructing noble metal alloy complex nanostructures with controllable synthetic method and excellent electrochemical property. Zhang et al. [295] focused on bimetallic NCs consisting of two distinct Pd and Pt and reviewed how to enhance their catalytic and electrocatalytic properties by tun- 
ing their specific structures. Our group emphasized the solution based method to controll the size, composition, and structure of bimetallic NCs [296]. Ferrando et al. [297] reviewed the alloy structure from the alloy clusters to NPs. In a molecular scale, they summarized the methods for generating nanoalloys, experimental techniques for characterization of nanoalloys, theoretical framework and computational methods for studying nanoalloys, and their properties. Wang's [298] and Hutchings's [299] groups separately reviewed the design of bimetallic catalysts for hydrogen generation and transformation of bio-renewable substances and reactions related to the realization of a bio-refinery. Table 1 summarizes the different shapes that have been achieved by reduction process.

In summary, the reduction process plays a vital role in the synthesis of monometallic and bimetallic NCs. We can achieve various shapes of metal NCs by using reducing agent, such as soluble solid, solvent, and gas. What's more, the reduction reaction is also an effective tool to synthesize polymetallic nanostructures. Recently, Yu's group [300] fabricated a carbon-supported $\mathrm{PtCo}_{2} \mathrm{Ni}_{2}$ trimetallic alloy through a simple reduction method, which showed a superior electrochemical activity towards oxygen reduction reaction.

\section{Decomposition reaction}

The decomposition methods usually consist of organometallic precursors, which will decompose at rigid conditions to generate the metastable nuclei or intermediates for further growth. Since the organometallic precursors mostly form homogeneous dispersions in solution, monodispersed NCs with high quality and narrow size distribution can be obtained by this strategy. To make sure the nucleation process driven by decomposition takes place at the same time, some approaches such as hot-injection method are usually used. For example, hot-injection technology can guarantee the burst nucleation and prevent the further nucleation.

\section{Decomposition for metals}

As the decomposition reaction usually takes place in high boiling-point organic solvent, the selection of metal precursor is very limited. Because the dominant form of metal precursor is water-soluble metal salts. A typical example is the unified approach to synthesize a large variety of NCs through a liquid-solid-solution (LSS) phase transfer and decomposition process [301]. As shown in Fig. 17, an ion exchange between the metal ions and sodium ions occurs spontaneously across the interface between sodium linoleate (solid) and the water-ethanol (solution) phase and results in the generation of noble metal linoleate. The primary reaction involved in LSS system is the decomposition of metal linoleate and further reduction at the liquid-solid or solution-solid interfaces. The as-generated metal NCs will be adsorbed by the linoleic acid and these NCs with long chains on the outside can be dissolved in the hydrophobic solvents. Actualy, this intermediate metal linoleate can be separately prepared and extracted for the further synthesis of metal NCs. Our group achieved the controlled synthesis of Ag NCs with size less than $4 \mathrm{~nm}$ by decomposition of Ag-oleate complexes in the presence of oleic acid and dodecylamine [302]. Another example is the formation and use of iron-oleate complexes in the controlled synthesis of octapod iron oxide, which was reported by Gao and co-workers [303]. However, since the reduction of iron requires much more rigid conditions, the decomposition of iron-oleate without the addition of strong reductant just leads to the generation of oxides. Most recently, Feng et al. [304] demonstrated that the thermolysis of Pd-thioate can be used to synthesize well-defined $\mathrm{Pd}$ and $\left[\mathrm{Pd}\left(\mathrm{SCH}_{3}\right)_{2}\right]_{6} \mathrm{NPs}$.

Since the reduction of late $3 \mathrm{~d}$ metals such as $\mathrm{Fe}, \mathrm{Co}$, and so on requires rigid conditions (additional reductant or high temperature), the selection of metal precursor prefers the metal complex with zero-valent metals. A work published in 2000 revealed the controlled synthesis of FePt bimetallic NCs can select the $\mathrm{Pt}(\mathrm{acac})_{2}$ (acac=acetylacetonate) and $\mathrm{Fe}(\mathrm{CO})_{5}$ as the metal precursors. The formation of FePt NCs with narraw size distribution and well defined shape involves the reduction of $\mathrm{Pt}(\mathrm{acac})_{2}$, decomposition

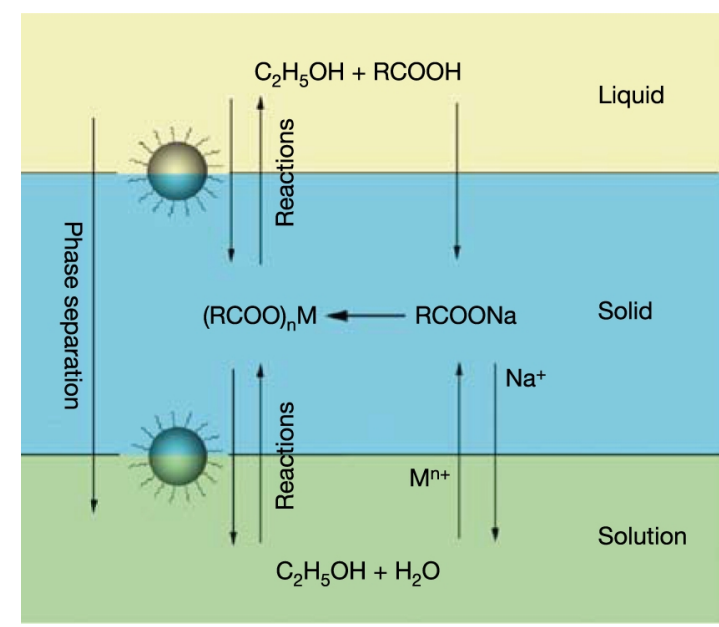

Figure 17 Scheme of LSS phase transfer synthetic strategy and the corresponding TEM images of the as-prepared Ag, Au and Ir NCs. Reproduced with permission from Ref. [301], Copyright 2005, Nature Publishing Group. 
of $\mathrm{Fe}(\mathrm{CO})_{5}$ and the alloying between Pt and Fe. The composition of FePt alloy can be modulated by varying the amounts of Fe and Pt precursors and the shape can be tuned by the addition of OA (oleic acid) and OAm stabilizers, and the control of nucleation rate [270,305-307]. The usage of carbonyl metal compound has been also discovered in the synthesis of Pt-Mn nanocubes [270]. In this study, Kang and Murray described the using of $\mathrm{Mn}_{2}(\mathrm{CO})_{10}$ can result in the generation of $\mathrm{Mn}(0)$, which is almost high valence state in air, via the decompositon process. The addition of $\mathrm{Mn}_{2}(\mathrm{CO})_{10}$ at elevated temperature during the heating process played a crucial role in the preparation of Pt-Mn alloy nanocubes. Moreover, the ligands of OA and OAm were found to be key to the shape dictating of nanocubes. The Pt-Mn alloy with a random element distribution of $\mathrm{Pt}$ and Mn was transferred to highly ordered intermetallic owing $\mathrm{L} 12$ phase $\left(\mathrm{AuCu}_{3}\right.$ structure) after a high temperature annealling. In a related study, Fang and co-workers [232] presented a versatile method for preparation of $\mathrm{Pt}-\mathrm{M}(\mathrm{M}=\mathrm{Fe}, \mathrm{Co}, \mathrm{Ni}, \mathrm{Cu})$ nanocubes. Afterwards, this synthetic methodology can be extended to the bimetallic alloy containing two late $3 \mathrm{~d}$ metals such as Fe-Co alloy. The bimetallic Fe-Co NCs were prepared by the co-decomposition of $\mathrm{Fe}(\mathrm{CO})_{5}$ and $\mathrm{Co}_{2}(\mathrm{CO})_{8}$ which were dissolved in tetrahydronaphthalene. To guarantee the composition of bimetallic can reflect the ratio in the precursor, Thanh and co-workers [308] developed a decomposition process via bimetallic carbonyl clusters. Because of the stable metalmetal bonds in the bimetallic carbonyl cluster anions such as $\left[\mathrm{FeCO}_{3}(\mathrm{CO})_{12}\right]^{-},\left[\mathrm{Fe}_{3} \mathrm{Pt}_{3}(\mathrm{CO})_{15}\right]^{2-},\left[\mathrm{FeNi}_{5}(\mathrm{CO})_{13}\right]^{2-}$, and $\left[\mathrm{Fe}_{4} \mathrm{Pt}(\mathrm{CO})_{16}\right]^{2-}$, the synthesis of bimetallic NCs can prevent the phase segregation and achieve the composition control reflecting the precursor.

\section{Decomposition for semiconductors}

Single precursor has been developed for the synthesis of monodispersed semiconductor NCs. The selected precursors should contain both the metal and corrsponding chalcogen elements, which can guarantee the success. The semiconductors can be prepared by directly decomposing the precursors under high temperature. The left or free ligands in solution also play a vital role in dictating the shape of final products. For example, $\left[\mathrm{M}_{10} \mathrm{Se}_{4}(\mathrm{SPh})_{16}\right]^{4-}$ $(\mathrm{M}=\mathrm{Cd}, \mathrm{Zn})$ were utilized by Strouse and co-workers [309] to synthesize monodispersed CdSe and ZnSe NCs in hexadecylamine solvent. Later on, Korgel and co-workers $[310,311]$ reported the solventless method to obtain $\mathrm{Cu}_{2} \mathrm{~S}$ $\mathrm{NCs}$ by mixing $\mathrm{Cu}$ salts and thiols to metal thiolate, which overcame the shortage that single precursor was usually hard to design and synthesize. Since the metal containing organometallic precursor is difficult to obtain, Murray et al. [312] developed the hot-injection method, during which the anion-containing part decomposed at high temperature and reacted with the pre-dissolved metal containing solution, to prepare CdSe NCs. The hot-injection method has been successfully generalized to high-quaity ZnSe, ZnS [313-315], PbS [316], PbSe, PbTe [317,318], InAs, InP $[319,320]$ and so on. For the effort in seeking "non-injection" methods for semiconductor NCs, Our group developed a facile "dispersion-decomposition" route to metal sulfide NCs with the help of alkanethiol [321] (Fig. 18). This method is carried out by employing simple inorganic metal salts as the starting reactants, which are easy to find and avoid the usage of toxic organometallic compounds. A long carbon chain alkanethiol, such as dodecanethiol, can be utilized as the phase transfer agents to drive to insoluble inorganic metal salts to form metal complex and dissolve in organic solution. In addition, the dodecanethiol can also serve as sulfur source due to the decomposition reaction, the ligand, and sometimes the solvent. The synthesis of semiconductor NCs undergoes

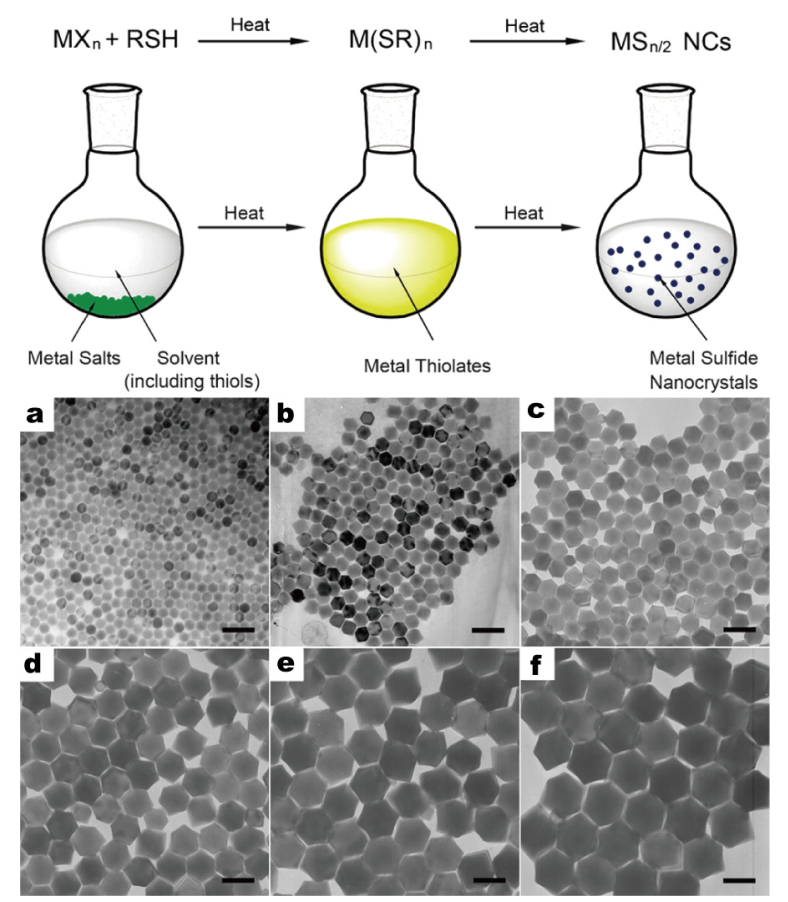

Figure 18 Scheme of the direct-heating "dispersion-decomposition" synthetic procedure and the TEM images of the octahedral PbS NCs obtained by using different amounts $\left(\mathrm{mol} \mathrm{L}^{-1}\right)$ of $\mathrm{Pb}(\mathrm{Ac})_{2} \cdot 3 \mathrm{H}_{2} \mathrm{O}$ : (a) 0.033 , (b) 0.050 , (c) 0.067 , (d) 0.100 , (e) 0.133 , and (f) 0.167 . All the scale bars are $100 \mathrm{~nm}$. Reproduced with permission from Ref. [321], Copyright 2011, WILEY-VCH Verlag GmbH \& Co. 
sequential dispersion and decomposition steps. First, the intermediate thiolate with low melting point is generated by the reaction between solid metal salt and alkanethiol, which can disperse in organic solvents homogeneously. The thiolate then decomposes into metal sulfide NCs such as $\mathrm{ZnS}, \mathrm{CdS}, \mathrm{PbS}, \mathrm{Ni}_{3} \mathrm{~S}_{4}, \mathrm{Ag}_{2} \mathrm{~S}$ and $\mathrm{Cu}_{2} \mathrm{~S}$ at a certain high temperature. The excess alkanethiol can also act as ligand which will bring the effect of shape-dictating in the growth of the NCs. Further, the control over size and shape can be achieved by varying the concentration of the reactants and oleic acid. For example, the size of PbS NCs can be easily controlled by the starting concentration of reactants. This finding provides a facile and general bottom-up method to generate a set of metal sulfide NCs. Utilizing simple inorganic metal salts as reactants, this strategy also avoids the injecting process with air-free protection during the synthesis, which is beneficial for the following large-scale synthesis.

In summary, the thermal decomposition method has largely been applied to the synthesis of functional nanomaterials such as semiconductor NCs, metals, oxides and so on. Systematically considering the appropriate reactants and ligands, the nucleation rate and decomposition temperature, the sizes, composition and morphologies of NCs can be well controlled. However, for resolving the problems such as toxic reactants and air-free reaction lines, large scale synthesis for commercial application still requires further improvement.

\section{TOP-DOWN STRATEGY}

Top-down strategies are mostly based on the bottom-up strategies and can be regarded as the carving of the pre-formed nanostructures which are obtained by the bottom-up strategies. For the example in physical method, the ball milling and lithographic is a powerful nonequilibrium processing tool to synthesize a variety of nanomaterials [322]. While physical top-down nanofabrication is capable of manufacturing nanoscale patterns on substrates, the chemical top-down approach has been proven to exhibit advantages in constructing more sophisticated nanostructures in solids and solutions. In this review, we only talk about the chemical top-down methods such as replacement, chemical etching, cation exchange, and exfoliation. In replacement reaction, an electron and material transfer between the bulk materials and free ions is used to direct the nanoscale features. For chemical etching, an oxidative etching which can remove the more reactive metals in bimetallic or polymetallic structure is involved, especially for the hollow or concave structures. For the cation ex- change, the exchange between two cations which is driven by precipitation equilibrium is utilized to obtain hybrid structures. For exfoliation method, the hierarchical structures which are made up by monomers can be delaminated into the monomers.

\section{Replacement reaction}

Galvanic replacement is a combination of oxidative and reductive processes, in which a metal transfers electrons to the ions of a second metal having higher reduction potential (see Table 2). Generally, a galvanic replacement reaction can be divided into two half reactions: one met-al losing electrons is corroded at the anode and the ions of the second metal are reduced, subsequently deposited at the cathode. For galvanic replacement reaction in solution, the template is oxidized and dissolved in solution, and then the ion of the second metal is reduced and deposited on the surface of the first metal. The driving force for the galvanic replacement reaction is the reduction potential difference between the two metallic species, and the first metal (which is often referred to as a sacrificial template) must have lower reduction potential than the second one. As such, when one piece of zinc ( $\mathrm{Zn}$ ) plate was immersed in $1 \mathrm{~mol} \mathrm{~L}^{-1} \mathrm{Cu}^{2+}$ aqueous solution, the zinc ( $\mathrm{Zn})$ starts to dissolve in the solution and the copper $(\mathrm{Cu})$ substance begins to form as a result of the reduction potential of $\mathrm{Cu}^{2+} / \mathrm{Cu}(0.34 \mathrm{~V}$ relative to the standard hydrogen electrode (SHE)) higher than $\mathrm{Zn}^{2+} / \mathrm{Zn}(-0.76 \mathrm{~V} v s$. SHE). This involves two half reactions: $\mathrm{Zn}-2 \mathrm{e}^{-} \rightarrow \mathrm{Zn}^{2+}, \mathrm{Cu}^{2+}+2 \mathrm{e}^{-} \rightarrow \mathrm{Cu}$. The rates of these two half reactions must be consistent to guarantee the electroneutrality of reaction system. Once the deposition of

Table 2 Reduction potentials of metals relative to the standard hydrogen electrode (SHE)

\begin{tabular}{cc}
\hline Reduction reaction & $E_{0}(\mathrm{~V}$ vs. SHE $)$ \\
\hline $\mathrm{CO}^{2+}+2 \mathrm{e}^{-} \rightarrow \mathrm{Co}$ & -0.28 \\
$\mathrm{Cu}^{2+}+2 \mathrm{e}^{-} \rightarrow \mathrm{Cu}$ & 0.34 \\
$\mathrm{Rh}^{3+}+3 \mathrm{e}^{-} \rightarrow \mathrm{Rh}$ & 0.76 \\
$\mathrm{Ag}^{+}+\mathrm{e}^{-} \rightarrow \mathrm{Ag}$ & 0.80 \\
$\mathrm{Pd}^{2+}+2 \mathrm{e}^{-} \rightarrow \mathrm{Pd}$ & 0.95 \\
$\mathrm{Ir}^{3+}+3 \mathrm{e}^{-} \rightarrow \mathrm{Ir}$ & 1.16 \\
$\mathrm{Pt}^{2+}+2 \mathrm{e}^{-} \rightarrow \mathrm{Pt}$ & 1.18 \\
$\mathrm{Au}^{3+}+3 \mathrm{e}^{-} \rightarrow \mathrm{Au}$ & 1.50 \\
\hline
\end{tabular}


copper fully covers the surface of the zinc substrate, the galvanic reaction is stopped by the compact and stable $\mathrm{Cu}$ surface. Significantly, the $\mathrm{Zn}^{2+} / \mathrm{Zn}$ and $\mathrm{Cu}^{2+} / \mathrm{Cu}$ system can also be applied to other noble metal systems.

This synthesis method can be applied to generate a number of nanostructures, including hollow, nanoframe, hetreojunction, core-shell, yolk-shell nanostructures and so on. Galvanic replacement in manipulating the properties of metal nanostructure is mainly from the following several aspects. 1) Composition. The elementary composition of the final product can be tuned by changing the amounts of precursor salts or sacrificial template. When more than one metal participate in galvanic replacement reaction, the spatial distributions of different elements can be controlled by changing the order of the addition for precursor salts [323]. 2) Internal structure. The atoms of the template are corroded and dissolved in the solution, which occurs in specific sites of the template due to different chemical reactivity or the coverage of the layer of the second metal. As a result, the outer shell of the template is mostly porous, and the thickness of the shell can be adjusted by the amount of the precursor salts. 3) Morphology. In general, the atoms which are reduced newly deposit on the surface of the template, so the final product type is similar to the template or just slightly altered. Overall, galvanic replacement provides a flexible and simple route to produce a number of advanced multifunctional nanostructures characterized by strictly controlled shapes and sizes, hollow interiors, porous walls and adjustable elemental compositions. What's more, galvanic replacement reaction does favor for core/shell or hollow nanostructure without additional reducing agent. To explain how galvanic replacement works in forming new nanostructures in detail, hollow and heterojunction structures are discussed as examples below.

\section{Replacement reaction for metals}

Hollow NCs have drawn much interest due to their potential application in catalytic, magnetics, electricity, and optics [324-327] and considerable efforts have been made in the synthesis of hollow NCs [326,328-333]. The galvanic replacement is an amazingly versatile and flexible route to synthesize hollow nanostructures with controllable compositions, wall thickness and pore structures. In general, these reactions involve an etching process which is driven by the difference in the electrochemical reduction potentials of two metallic species and accompanied with the Kirkendall effect [326,334-336]. The inner hollow is generated by oxidative dissolution of the metal NCs which are used as templates. For example, Chen and co-workers
[337] reported $\mathrm{Ag}$ nanocubes transformed into $\mathrm{Pd}-\mathrm{Ag}$ nanoboxes by adding $\mathrm{Na}_{2} \mathrm{PdCl}_{4}$ via replacement reaction, which depended on the different reduction potentials between the two metals $\left(\mathrm{Ag}^{+} / \mathrm{Ag} 0.8 \mathrm{~V}\right.$ vs. $\left.\mathrm{Pd}^{2+} / \mathrm{Pd} 0.95 \mathrm{~V}\right)$. By tuning the amount of $\mathrm{Na}_{2} \mathrm{PdCl}_{4}$ or $\mathrm{Na}_{2} \mathrm{PtCl}_{4}$ salt added, they could control the extent of Ag nanocubes (sacrificial template) etched and the product morphology (Fig. 19). The replacement reaction usually occurs on the surface of the Ag nanocubes, primarily at the edge atoms [338]. The atomic step edges necessarily presenting at the corners may provide the most reactive sites for initiation of pitting at this location [339]. During replacement reaction, Ag atoms from the interior rather than the surface of the $\mathrm{Ag}$ nanocube are oxidated, due to the $\mathrm{Pd}$ atoms reduced and deposited on the surface of the template, therefore further blocking the dissolution of the surficial Ag atoms. It is known that $\mathrm{Pd}$ and $\mathrm{Ag}$ atoms can form $\mathrm{Pd}-\mathrm{Ag}$ binary alloy [340], plating of Pd on the surface will result in the formation of walls composed of Pd-Ag alloy. In addition to $\mathrm{Pd}$-Ag nanoboxes, other noble metal nanoboxes ( $\mathrm{Au}-\mathrm{Ag}$ [341], Pt-Ag [342]) have also been synthesized.

For the synthesis of metal hollow structure, Sun and Xia [326] gained the first breakthrough, during which $\mathrm{Au}$ nanoboxes were prepared by the replacement of $\mathrm{Ag}$ nanocube with $\mathrm{HAuCl}_{4}$. They further reviewed the synthesis of Au nanocages and their biomedical applications [343]. Recently, González and co-workers [344] combined the galvanic exchange and Kirkendall growth to achive the

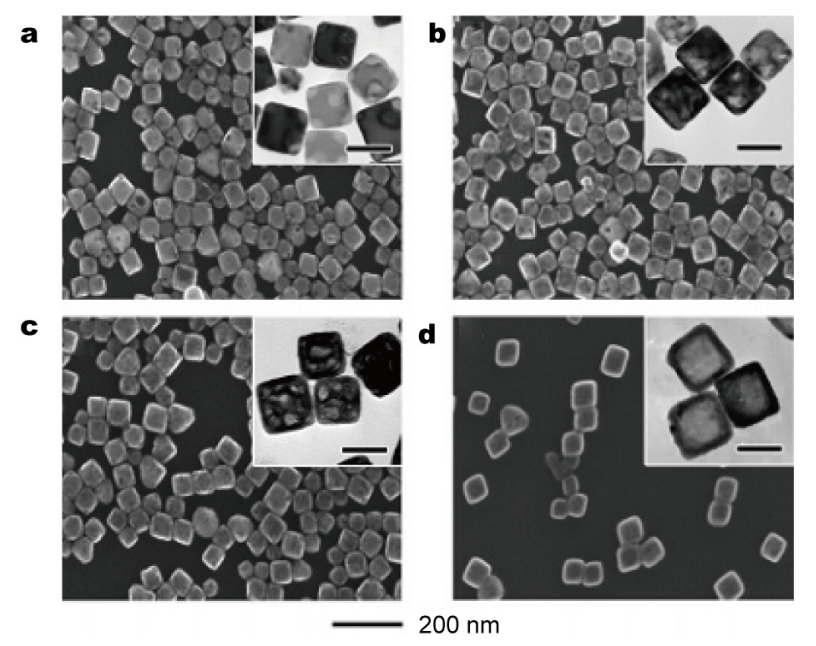

Figure 19 Formation of Ag-Pd nanoboxes through galvanic replacement between $\mathrm{Ag}$ nanocubes and $\mathrm{Na}_{2} \mathrm{PdCl}_{4}$. The SEM images were taken after reacting $\mathrm{Ag}$ nanocubes with (a) 0.3 , (b) 0.6 , (c) 0.9 , and (d) $1.5 \mathrm{~mL}$ of a 0.5 mmol L-1 aqueous solution of $\mathrm{Na}_{2} \mathrm{PdCl}_{4}$. Insets are corresponding TEM images with a $50 \mathrm{~nm}$ scale bar. Reproduced with permission from Ref. [337], Copyright 2005, American Chemical Society. 
carving at nanoscale. They provided a synthetic route for the production of polymetallic hollow structures with different morphologies and compositons, controlled by the simultaneous or sequential action of galvanic replacement and the Kirkendall effect.

\section{Replacement reaction for oxides}

The galvanic replacement can also occur in oxide NCs and produce hollow oxide nanostructures. Oh and co-workers [345] recently synthesized the hollow box-shaped NCs of $\mathrm{Mn}_{3} \mathrm{O}_{4} / \gamma-\mathrm{Fe}_{2} \mathrm{O}_{3}$ ("nanoboxes") and transferred these nanoboxes into hollow cagelike $\mathrm{Fe}_{2} \mathrm{O}_{3}$ ("nanocages") via replacement reaction. The replacement reaction shown in this study was promoted by the difference in the standard reduction potentials of $\mathrm{Fe}^{3+} / \mathrm{Fe}^{2+}(0.77 \mathrm{~V})$ and $\mathrm{Mn}_{3} \mathrm{O}_{4} / \mathrm{Mn}^{2+}$ $(1.82 \mathrm{~V})$ pairs, in which $\mathrm{Fe}^{2+}$ ions reduced $\mathrm{Mn}^{3+}$ ions in the $\mathrm{Mn}_{3} \mathrm{O}_{4}$ NCs (Fig. 20). When the galvanic reaction started, the atoms on the surface of $\mathrm{Mn}_{3} \mathrm{O}_{4}$ were initially oxided and dissolved into the solution, and thereby $\gamma-\mathrm{Fe}_{2} \mathrm{O}_{3}$ was reduced and deposited on the $\mathrm{Mn}_{3} \mathrm{O}_{4}$ surface, which prevented outward $\mathrm{Mn}^{2+}$ diffusion. This transformation preferentially happened at the edge atoms of $\mathrm{Mn}_{3} \mathrm{O}_{4} \mathrm{NCs}$ [332] (Fig. 20g, inset). This was because a galvanic replacement would be initiated immediately at the site with the highest surface energy (e.g., defect, stacking fault, or step) $[66,346,347]$. The increase in the number of empty octahedral sites caused by the dissolution of the reduced $\mathrm{Mn}^{2+}$ could result in the complete disruption of the remaining lattice which consisted of tetrahedral $\mathrm{Mn}^{2+}$ and oxygen anions [348]. This process generated pinholes which were not covered by the reduced $\mathrm{g}-\mathrm{Fe}_{2} \mathrm{O}_{3}$ layer at the initial stage of the reaction. Pores were developed inside the NCs by a mechanism comparable to pinhole corrosion, in which the pinholes were used as transport paths during the dissolution of the $\mathrm{Mn}_{3} \mathrm{O}_{4}$ core. As the reaction progresses, the electrons released from the $\mathrm{Fe}^{2+}$ migrate inward and reduce the octahedral $\mathrm{Mn}^{3+}$ in the interior. Along with the increase of surface deposition of $\mathrm{g}-\mathrm{Fe}_{2} \mathrm{O}_{3}$, it maked the
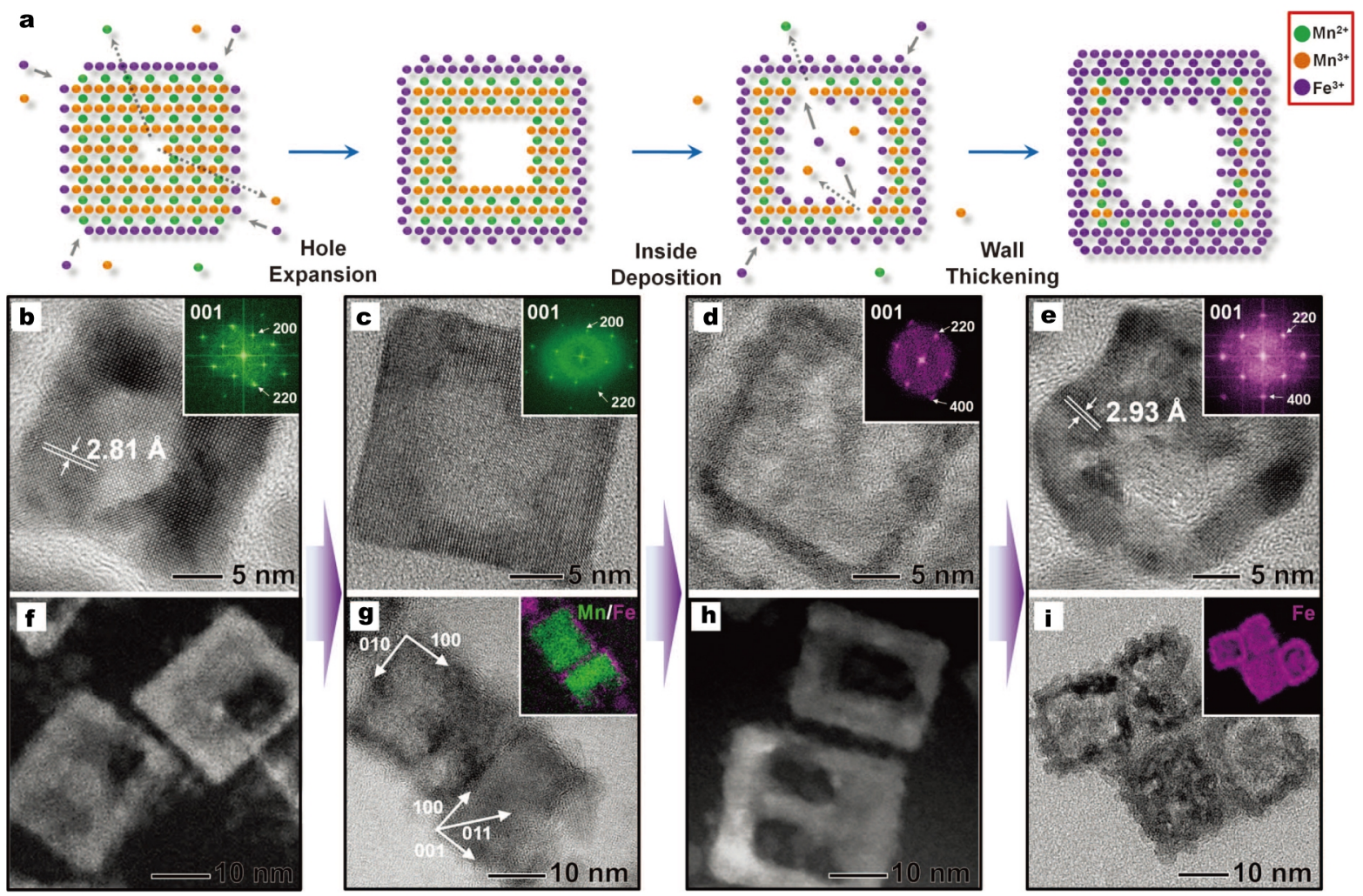

Figure 20 (a) Schematic illustration of the transformation of $\mathrm{Mn}_{3} \mathrm{O}_{4} \mathrm{NCs}$, showing the evolution of their morphology via the localized dissolution of $\mathrm{Mn}_{3} \mathrm{O}_{4}$ and the surface precipitation of $\mathrm{g}-\mathrm{Fe}_{2} \mathrm{O}_{3}$. (b-e) HRTEM images of the hollow nanostructures synthesized by the reaction of $\mathrm{Mn}_{3} \mathrm{O}_{4} \mathrm{NCs}$ with 1 $\mathrm{mL}$ of aqueous solutions of iron(II) perchlorate having different concentrate ions: (b) 0.4, (c ) 0.6, (d) 1.0, and (e) $1.6 \mathrm{~mol} \mathrm{~L}^{-1}$. Insets show the corresponding FT patterns. (f) High-angle annular dark-field scanning TEM (HAADF- STEM) image of the nanoboxes shown in (b). (g) TEM image and a corresponding energy filtered TEM (EFTEM) image of the nanoboxes shown in (c). (h) HAADF-STEM image of the nanocages shown in (d). (i) TEM image and a corresponding EFTEM image of the nanocages shown in (e). Reproduced with permission from Ref. [345], Copyright 2013, the American Association for the Advancement of Science. 
dissolution rate of $\mathrm{Mn}_{3} \mathrm{O}_{4}$ slow down by hindering the diffusion of ions and electrons to its surface [349]. The authors also found if the volume of $\mathrm{Mn}_{3} \mathrm{O}_{4}$ was below a certain critical limit, its complete dissolution and thus the nearly total replacement of $\mathrm{Mn}$ by Fe could be achieved. When a less concentrated solution was used, the cores of the $\mathrm{Mn}_{3} \mathrm{O}_{4} \mathrm{NCs}$ were dissolved partially, and the nanoboxes with relatively thick walls were formed (Figs 20b, c, f and g). It should be pointed out that, in addition to hollow binary nanoboxes and oxide nanostructures, galvanic replacement could also be applied to generate hollow nanotubes [329,350,351], nanocages [352-354], multiple-walled nanoshell [355] and so on. Most recently, Yang and co-workers [356] reported the galvanic replacement of $\mathrm{Ru}$ and a set of transition metals such as $\mathrm{Co}, \mathrm{Cu}, \mathrm{Fe}$ and $\mathrm{Ni}$ to fabricate hollow nanostructures. Moreover, after a simple thermal treatment in air, the bimetallic structures can be in-situ transformed into the hollow metal oxides structures without changing their symmetry.

\section{Replacement reaction for heterojunction structures}

Heterojunction nanostructures constructed with different building blocks of nanoscale dimensions have attracted special attention owing to their unique and tailored properties for various applications in materials science [357-364]. The galvanic replacement is a straight-forward method to prepare heterojunction nanomaterials through partial replacement of one metal NP with another metal component $[365,366]$. Galvanic replacement between oxidizable metal substances and reducible ions in the solution has been reported [365,366]. For example, Kim and co-workers [365] successfully synthesized $\mathrm{Pt} / \mathrm{Mn}_{3} \mathrm{O}_{4}$ heterojunction structure via employing the galvanic replacement process happening between the $\mathrm{Mn}_{3} \mathrm{O}_{4}$ surface and $\mathrm{PtCl}_{4}{ }^{2-}$ complex (Fig. 21). In this reaction, $\mathrm{PtCl}_{4}{ }^{2-}$ ions were spontaneously reduced to $\mathrm{Pt}$ atoms deposited on the surface of $\mathrm{Mn}_{3} \mathrm{O}_{4}$ $\mathrm{NCs}$, and thereby part of the $\mathrm{Mn}_{3} \mathrm{O}_{4}$ particles were oxidized and dissolved into the solution resulting in $\mathrm{Pt} / \mathrm{Mn}_{3} \mathrm{O}_{4}$ heterojunction structure. The driving force of the reaction is different reduction potentials between $\mathrm{PtCl}_{4}{ }^{2-}(0.758$ $\mathrm{V})$ and $\mathrm{Mn}_{3} \mathrm{O}_{4}(0.712 \mathrm{~V})$. The reactions involved are as follows:

$$
\begin{gathered}
\mathrm{PtCl}_{4}^{2-}+2 \mathrm{e}^{-} \rightarrow \mathrm{Pt}(\mathrm{s})+4 \mathrm{Cl}^{-}, \\
\mathrm{Mn}_{3} \mathrm{O}_{4}(\mathrm{~s})-\mathrm{e}^{-}+8 \mathrm{H}^{+} \rightarrow 3 \mathrm{Mn}^{3+}+4 \mathrm{H}_{2} \mathrm{O}, \\
\mathrm{PtCl}_{4}^{2-}+2 \mathrm{Mn}_{3} \mathrm{O}_{4}(\mathrm{~s})+16 \mathrm{H}^{+} \rightarrow \mathrm{Pt}(\mathrm{s})+6 \mathrm{Mn}^{3+}+4 \mathrm{Cl}^{-}+8 \mathrm{H}_{2} \mathrm{O} .
\end{gathered}
$$

The method explored in this study can be further applied

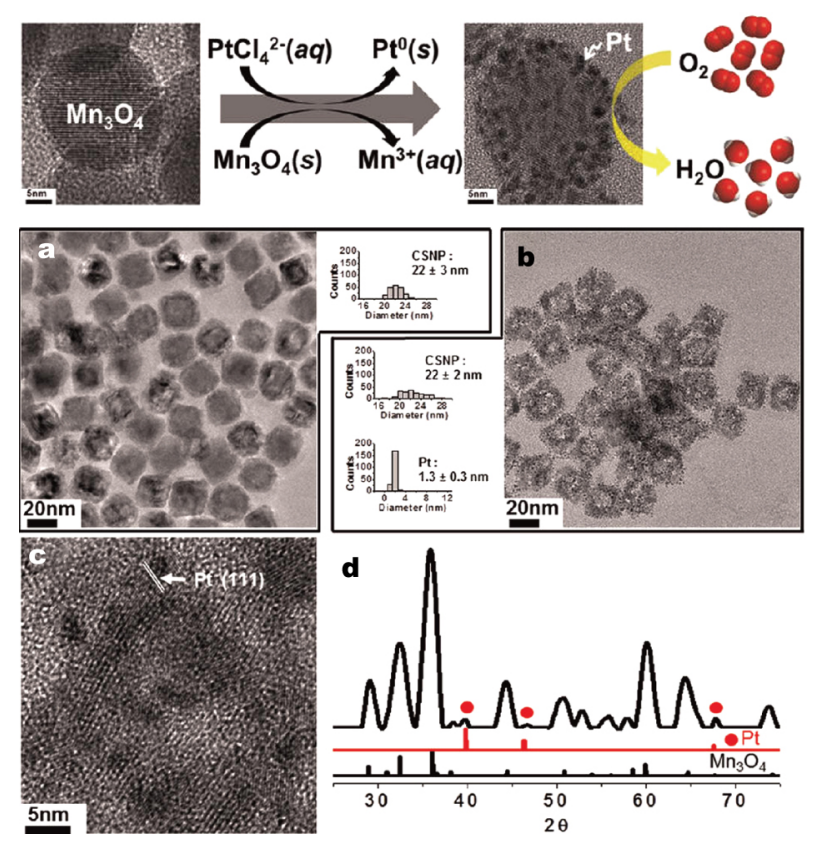

Figure 21 The top is the scheme illustrating the replacement reaction between $\mathrm{Mn}_{3} \mathrm{O}_{4}$ and $\mathrm{PtCl}_{4}^{2-}$. (a and b) TEM and size distribution histogram, (c) HRTEM image of hybrid structures, and (d) XRD pattern of NPs obtained by treating $\mathrm{Mn}_{3} \mathrm{O}_{4} \mathrm{NCs}$ in a $\mathrm{Na}_{2} \mathrm{PtCl}_{4}$ solution at $70^{\circ} \mathrm{C}$ for 30 min. Reproduced with permission from Ref. [365], Copyright 2013, American Chemical Society.

to $\mathrm{PdCl}_{4}{ }^{2-}$ system. In addition, galvanic replacement method was subsequently also applied to synthesize $\mathrm{Au} / \mathrm{Cu}_{2} \mathrm{O}$ heterojunction nanostructure by Xiong and co-workers [366].

\section{Chemical etching}

In the past decade, by altering a number of experimental parameters (e.g., metal precursor, reducing agent, temperature, solvent, and capping agent), the nucleation and growth of metal NCs and the crystallinity and surface facets of NCs can be manipulated [2,367-369]. Apart from the parameter control for the bottom-up synthesis, preserving the geometry of NCs and refining their microstructure through top-down strategy represent another significant challenge to nanoscience. For instance, extensive studies have demonstrated that the external stimulus can cause the dynamic (and sometimes reversible) shape changes of a metal NC in the ambient environment. Among the various environmental factors, oxidative etching has been proven as an effective tool to induce the structural changes [77,370-376]. In addition to the negative role such as metal loss, the oxidative etching can be also recognized as a facile and moderate strategy to access new structure under 
appropriate condition. Basically there are two complementary approaches for the synthesis of NCs: dissolution and growth. During the growth stage, the morphology of NCs is usually associated with the equilibrium between dissolution and growth rates at specific directions. When the synthesis is conducted in air, together with the presence of a proper coordination ligand for the metal ions, the zero-valent species (including atoms, clusters, and seeds) can all be possibly oxidized back to the ionic states which will alter the nucleation and growth modes [377,378]. As a result, oxidative etching can serve as an effective and robust tool for reshaping NCs via atomic addition and subtraction.

The essence of oxidative etching is the oxidation of zerovalent atoms, clusters, seeds to ionic states in the presence of oxidative species such as $\mathrm{O}_{2}$, halide ions, and iron(III) in solution. In our daily life, oxidative etching is a common phenomenon. A typical case is corrosion that when steel is in contact with both air and water, the rust formation will begin to occur at the surface of steel. Similarly, oxidative etching caused by the impurities reagent and oxygen in air has an inevitable influence on the synthesis, and thus it plays a vital role during NC synthesis. For example, the synthesis of Ag NCs involves the reduction of a salt precursor by a polyol process [241].

EG serves as solvent and reducing agent containing chloride and ferric impurities, both of which can corrode metals. The presence of $\mathrm{Cl}^{-}$ions, combined with oxygen from air, often results in significant changes to the crystallinity and morphology of the final products [379].

Since the oxidative etching was found to have impact on shape-controlled metal nanostructure synthesis in 2004, the chemical etching approach has become an effective and elegant "top-down" method to precisely control over the nucleation and growth of metal NCs at the atomic level in solution. Subsequently, oxidative etching has attracted extensive attention and significant efforts have been made to expand and apply this method in the synthesis of NCs. Oxidative etching is capable to modify the shapes and structures of NCs via atomic subtraction and addition.

\section{Shape control}

The shape of a NC has an impregnable correlation with the interfacial energy of different surface facets. The formation of surface facets is determined by total interfacial free energy to make the final structure more thermodynamically favored. Adding suitable capping agent on to the surface is a useful tool to tailor the shape of NCs which can modify their surface energy. In general, the surface energies for fcc metals with three low-index facets follow the sequence of $\gamma(111)<\gamma(100)<\gamma(110)$. Owing to the term of the different surface energy, oxidative etching is a useful method to show specific selectivity toward different facets. Based on thermodynamics, atoms are expected to nucleate and grow into cuboctahedra (with a nearly spherical shape) composed by (111) and (100) facets to minimize the total surface energy [380-382]. In a solution-phase process, the surface free energies can be altered by adsorption of impurities or capping agents, and thus new shapes will be induced. Xiong et al. [77] demonstrated a simple and versatile method for promoting the formation of Pd single-crystal nanorods composed of (100) and (110) facets as side surfaces by introducing bromide into the reaction solution. In this process, bromide was regarded as a crucial role in the formation of nanostructures enclosed by the (100) and (110) side facets. One side of single-crystal seeds was selectively activated by oxidative etching while certain species passivated the surface of the seeds thus inducing their anisotropic growth in this work. This principle was also demonstrated by using a mixture of $\mathrm{NH}_{4} \mathrm{OH}$ and $\mathrm{H}_{2} \mathrm{O}_{2}$ to etch single-crystal Ag NPs [199]. It was noted that the $\{100\}$ facets of Ag NCs exhibited slower etching rate than that on the $\{111\}$ facets. Owing to this, the morphology of Ag octahedra successfully evolved to concave octahedra, eventually yielded octapods by the higher concentration etchant (Fig. 22).

In general, oxidative etching of NCs is inclined to initially occur from sites with sharp features on the surface. These sites with rich low-coordination atoms are more sensitive to the oxidative etching. Repairing the atoms at these sites can significantly reduce the total free energy, thus making the final structure thermodynamics dominated. For instance, our group presented a controllable "top-down" synthesis of concave Pt-Ni alloys which exhibited higher catalytic activity compared to the uncorroded alloys through a coordination assisted chemical-etching process (Fig. 23) [383]. Coordinating agent (dimethylglyoxime) with the presence of oxygen was used to control the chemical etching process at room temperature to synthesize a concave structure. The dimethylglyoxime, which was a selective binding agent to $\mathrm{Ni}$, can promote the transformation of zero-valent $\mathrm{Ni}$ to $\mathrm{Ni}^{2+}$ by the oxygen in air. This oxidative etching promoted by coordination was demonstrated by the etching of octahedral Ni-rich Pt-Ni alloys, which can be finally eroded to concave octahedral $\mathrm{Pt}_{3} \mathrm{Ni}$. Octahedral $\mathrm{PtNi}_{10}$ was utilized as a probe to elucidate the morphological evolution during the etching process. The etching process initially started at the corners, then the edges and the facets were excavated to curved cavities and finally the concave structure was gen- 

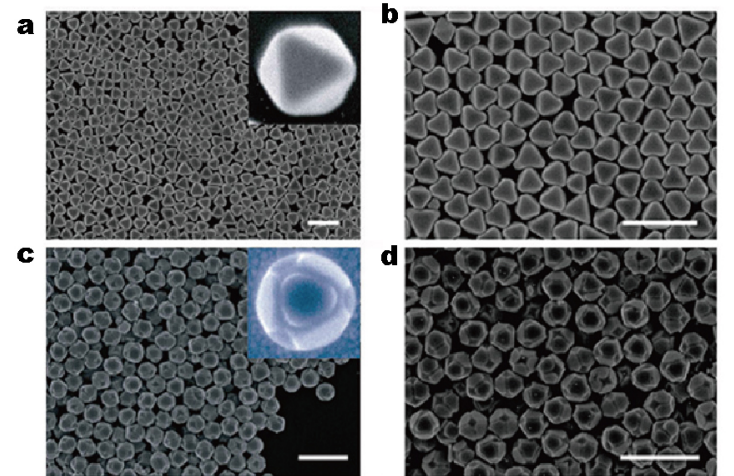

e

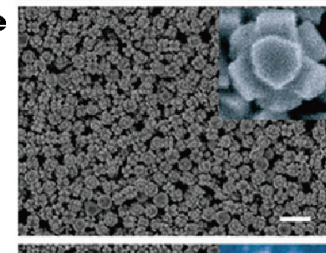

$\mathbf{g}$
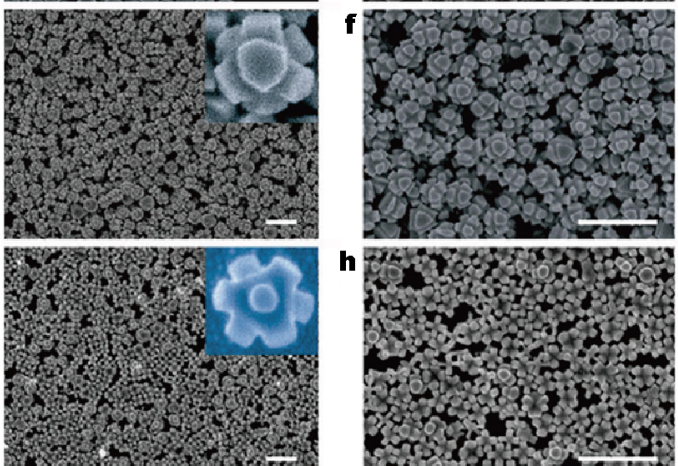

h

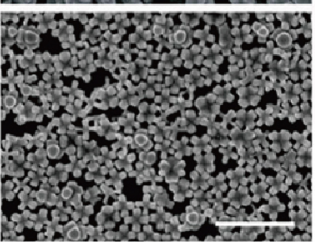

i

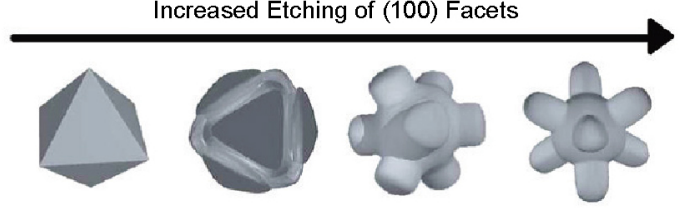

Figure 22 SEM images of the Ag NPs: (a, b) octahedra-shaped starting material; (c, d) using a small amount of etchant; (e, f) a slight higher concentration of etching solution; $(\mathrm{g}, \mathrm{h})$ relatively high concentration of etching solution. (i) Scheme showing the effects of selectively etching the $\{100\}$ faces of Ag octahedra. All scale bars shown represent $1 \mu \mathrm{m}$. Reproduced with permission from Ref. [199], Copyright 2009, American Chemical Society.

erated. By implementing a similar strategy, another type of Pt-Ni alloy with a predictable concave cubic morphology was also obtained.

Hollow metal nanostructures with increased surface area and hollow interior always show exceptional chemical and physical properties compared to their solid structures [373]. Oxidative etching is helpful to create hollow nanostructures by removing the interior of solid NCs [353]. Single-crystal Pd nanoboxes and nanocages could be obtained via the selective removal of internal Pd from Pd nanocubes by etchant without the involvement of exotic templates [384]. Pitting corrosion was used in this procedure and six representative stages were observed: reduction of $\mathrm{PdCl}_{4}{ }^{2-}$ to form $\mathrm{Pd}$ nanocubes with slightly

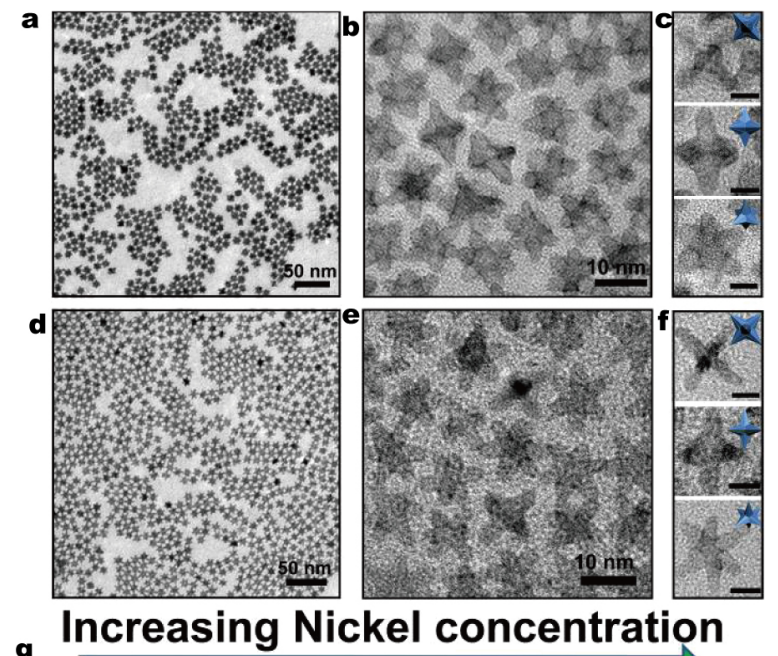

g

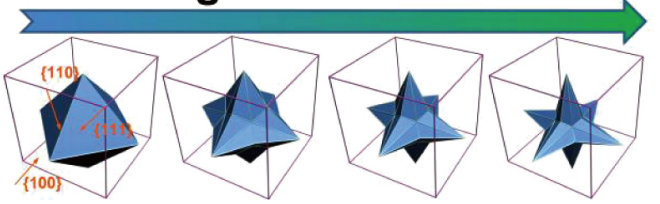

Figure 23 TEM images of (a) corroded $\mathrm{PtNi}_{3}$ and (d) corroded $\mathrm{PtNi}_{10}$. Magnified TEM images of (b) corroded $\mathrm{PtNi}_{3}$ and (e) corroded $\mathrm{PtNi}_{10}$. Panels (c) and (f) are the HRTEM images and corresponding models orientated along $\{100\}$ (top panel), $\{110\}$ (middle panel), and $\{111\}$ (bottom panel) directions, respectively (scale bars are $5 \mathrm{~nm}$ ). (g) Scheme illustration showing the evolution in NPs shape as a function of Ni:Pt mole ratio in the original Ni-rich alloys. Reproduced with permission from Ref. [383], Copyright 2012, WILEY-VCH Verlag GmbH \& Co.

truncated corners; oxidative etching surpasses the reduction process over the Pd nanocubes; the interior of nanocubes becomes more empty and the defects on the surface shrink to generate the hollow box; the inner voids further connect with each other to form bigger hollow interior inside the nanocube and the hole in the surface finally disappears; small holes start to appear at the corners to turn the nanobox into nanocage; the nanocage is finally transferred into a ring due to the additional etching and Ostwald ripening. This approach can also be implemented to produce monometallic NCs by selectively removing one of the metals from binary NCs. For example, Rh cubic nanoframes could be produced by selectively removing the cores of $\mathrm{Pd}-\mathrm{Rh}$ concave nanocubes with an aqueous etchant containing $\mathrm{Fe}^{3+}$ species [376]. Additionally, $\mathrm{Ag}$ can be selectively removed from $\mathrm{Au}-\mathrm{Ag}$ nanoboxes to leave $\mathrm{Au}$ cubic nanocages and nanoframes with the combined action of $\mathrm{Fe}\left(\mathrm{NO}_{3}\right)_{3}, \mathrm{NH}_{4} \mathrm{OH}$, or $\mathrm{H}_{2} \mathrm{O}_{2}[341,385]$. Oxidative etching serves as a simple and elegant method for the fabrication of hollow nanostructures. 


\section{Size control}

Particle size is one of the most crucial factors strongly related to the chemical and physical properties of nanomaterials. For instance, surface plasmonic features of $\mathrm{Ag}$ and $\mathrm{Au}$ NCs can be controlled by tuning their sizes. Therefore, considerable efforts have been made to tune the particle size to obtain excellent properties in various synthetic systems. Adjusting the synthesis temperature and the concentration of metal ions are useful tools to control the particle size $[109,250]$. These existing methods are unable to tune particles size in a wide range. The etching can also be employed as a tool to control the particle size in the nucleation stage. Oxidative etching has multiple functions to tune the particle size. In a reductive environment, the precursor ions oxidized from the initially formed seeds will be subsequently reduced to metal atoms and diffused in or deposited on the remaining seeds, finally increasing their size. The method of oxidative etching is an effective tool to control the particle size with consistent geometrical shapes. In a typical process, Xiong et al. [192] synthesized Pd nanocubes with different size dependent on the added concentration of $\mathrm{FeCl}_{3}$ by using the same concentration of Pd precursor. In similar strategy, they also demonstrated that $\mathrm{HCl}$ can serve as an effective etchant during the nucleation stage to control the size. The sizes of Pd nanocubes could be tuned from 8 to $20 \mathrm{~nm}$ in an aqueous synthesis when the concentration of $\mathrm{HCl}$ was increased (Fig. 24). This similar mechanism was also applied to tune the size of Pd cuboctahedra from 5 to $20 \mathrm{~nm}$ [386]. It is noted that the geometrical shapes of products can be kept consistent in this size controlled method, allowing researching their size-dependent performance and further applications.

\section{Structure control}

In a typical synthesis of NCs, the seeds derived from nuclei play a dominant role in the final resultant NCs. Oxidative etching has an effective function on each stage. The crystallinity of the NC (single crystal versus twinned) is determined by the structure of seeds obtained from the nucleation and evolution stages. Therefore, oxidative etching is capable of manipulating the synthesis of multiply twinned to single-crystal structure during the reaction in several systems. For instance, in a polyol synthesis of Ag NCs, Ag NPs with twin defects were selectively dissolved by employing $\mathrm{Cl}^{-}$and air as etchants, resulting in high yields of single-crystal seeds [387]. By replacing $\mathrm{Cl}^{-}$with $\mathrm{Br}^{-}$, the single twin seeds was dominant to facilitate the formation of right bipyramids due to the etching ability of $\mathrm{Br}^{-}$was weaker

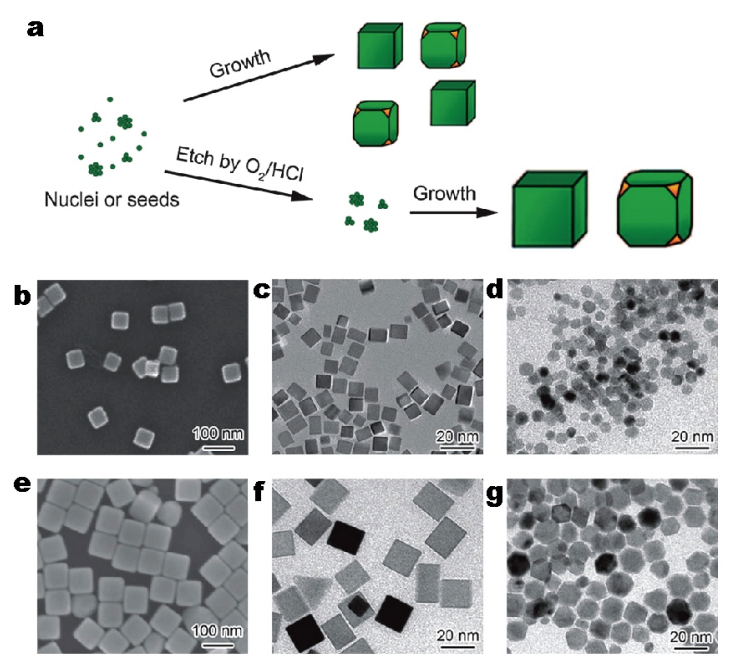

Figure 24 (a) Schematic illustration for size control of metal nanocubes via oxidative etching. SEM images of Ag nanocubes prepared in EG in the presence of different amounts of $\mathrm{HCl}$ : (b) 0.21 and (c) $0.28 \mathrm{mmol} \mathrm{L}^{-1}$. TEM images of Pd nanocubes prepared in water in the presence of $\mathrm{HCl}$ at different concentrations: (d) 0 and (e) $109 \mathrm{mmol} \mathrm{L}^{-1}$. TEM images of $\mathrm{Pd}$ cuboctahedra prepared in water in the presence of $\mathrm{HCl}$ at different concentrations: (f) 0 and (g) $218 \mathrm{mmol} \mathrm{L}^{-1}$. Reproduced with permission from Ref. [386], Copyright 2012, WILEY-VCH Verlag GmbH \& Co.

than that of $\mathrm{Cl}^{-}$. High yields of the single twinned seeds ensure the further growth of right bipyramids in the presence of $\mathrm{Br}^{-}$(Fig. 25). The $\mathrm{Br}^{-}$are relatively weak to completely prevent those seeds with only one twin defect compared to $\mathrm{Cl}^{-}$, thus having a remarkable effect on etching the seeds with multiple twin defects to remain single-twinned structures.

In summary, oxidative etching has been employed as an effective and convenient tool in the synthesis of metal NCs. Considerable progress has been made to control the shape, size and structure of metal NCs by using oxidative etching. The NCs obtained by this method have been applied in physical and chemical fields. However, when needed, it is essential to diminish or eliminate the role of oxidative etching in the reaction system $[388,389]$. In short, if controlled appropriately, oxidative etching provides accessibility and possibility for synthesizing NCs and refining their function for application.

\section{Cation exchange}

Cation exchange reaction can be defined as the process involving the replacement of cations in a NC without changing the anionic part. The overall cation exchange reaction is $\mathrm{A}^{n+}($ liquid $)+\mathrm{B}-\mathrm{C}(\mathrm{NC})=\mathrm{B}^{n+}($ liquid $)+\mathrm{A}-\mathrm{C}(\mathrm{NC})$. This 

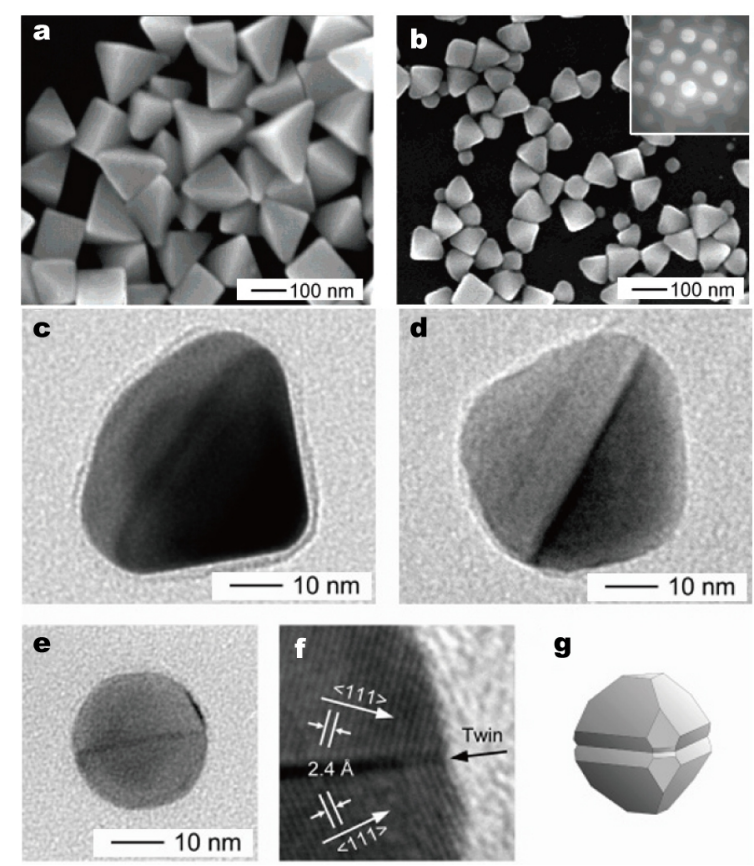

$\mathbf{g}$

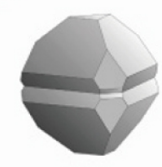

Figure 25 SEM images of bipyramids approximately (a) $150 \mathrm{~nm}$ and (b) $75 \mathrm{~nm}$ in edge length. (c) TEM of a right bipyramid $40 \mathrm{~nm}$ in edge length taken from the reaction at $t=2.5 \mathrm{~h}$. An internal difference in contrast suggests that a twin plane bisects its two halves. (d) The twin plane that bisects the bipyramid is clearly visible after tilting the same particle by $45^{\circ}$. (e) At $t=1.5 \mathrm{~h}$, single twinned seeds approximately $25 \mathrm{~nm}$ in diameter were observed. (f) HRTEM of a twinned seed shows the lattice fringes reflecting across the (111) twin plane. (g) Model of the seed in (c) showing (111) truncation of corners, reentrant (111) surfaces at the twin boundary and reentrant (100) surfaces at the twin boundary corners. Reproduced with permission from Ref. [253], Copyright 2006, American Chemical Society.

process is driven by the different equilibrium constants of precipitation and started by exposing the parent ionic crystal to other cations. The new cations will replace the parent crystal at the same time and the initial cations diffuse out, which occurs either in solid or solvated liquid form. In the equation, A means the ingoing metal ion, B and $\mathrm{C}$ mean the cation and anion in the parent NC. It is unusual in thermodynamic equilibrium for exchange reaction at room temperature, so that the outcome of the reaction is mostly determined by the nucleation energy barrier. Usually, the ion exchange takes place between the dissolution and re-precipitation processes simultaneously in an adjacent space, allowing mass communication between the parent and product crystals [390-393].

In tradition, the development of cation exchange has undergone two distinct stages which are the bulk materials and nanomaterials. Several thermodynamic factors should be considered in the cation exchange process, which are crystal energies related to the dissociation of $\mathrm{C}-\mathrm{A}$ and the association of M-A, desolvation and solvation energies of the cations $\mathrm{M}$ and $\mathrm{C}$. It is not difficult to find out that the more strongly the parent cation can be solvated in the reaction solvent than the ingoing cation, the more easily the whole cation exchange can take place. The prediction of the thermodynamic information involved can aid the judgement of likelihood of cation exchange process. For instance, the isovalent exchange may be dictated more by the lattice energy and ionic size considerations. In contrast, the nonequivalent exchange such as exchange between the $\mathrm{Cu}^{+}$ and CdS is strongly dominated by the solvation difference between the two ions. In the cases that influence from the lattice or solvation is negligible, the intrinsic law of mass action for each elementary reaction and precipitation equilibrium can be used to drive the cation exchange.

In fact, ion exchange in newly formed phase is also influenced by the kinetic factors. Since each sub-reaction is linked to the cation exchange process, all of the activation energies would determine the inherent outcome of the reaction. The cation exchange rate can be accelerated by the increasing of the temperature, though the stability or manufacturability of the material would be obviously reduced. For the bulk materials, the cation exchange usually lasts for a long time stage. That is, the early stage usually may last for several days at the temperature of $100-800^{\circ} \mathrm{C}$. On the premise of preserving the materials' stability, the reaction temperature should be elevated as high as possible to guarantee the following cation exchange process under thermodynamic equilibrium, preventing the generation of metastable or non-equilibrium structures.

Under nanometer scale, the kinetics roles will hold the advantage for the cation exchange reaction due to the dramatically increased surface area and high reactivity. For the NCs, the cation exchange reactions can be likened to the molecular reaction rather than the solid state, which may be two orders of magnitude faster than that for bulk materials. Hence, the reaction at room-temperature becomes easier to occur and the reaction parameters will be largely afffected by the sizes, compositions, and morphologies [394]. More importantly, the high surface area and reduced subreaction barriers resulting from the NCs are beneficial for the full cation exchange between the two cations, enabling more possibility to access metastable phases. In this situation, the reorganizations, strain relief and more complexity can be anticipated for the cation exchange in nanoscale.

\section{Coulombic repulsive force}

If the phase remains unchanged in the presence of other cations, the Coulombic repulsive force will be generated 
between these two cations. Actually, the Coulombic repulsive force is similar to the competition of two cations in the cation exchange except for no phase change taking place. This effect does have important effect on the controlled synthesis of many metal-chalcogenide structures. For instance, in a reaction system containing both $\mathrm{Au}^{3+}$ and $\mathrm{Cd}^{2+}$, the cationic repulsive force prevented the nucleation of gold on cadmium-rich end. As a result, gold nucleated and grew preferentially at sulfur-rich end, generating a unique $\mathrm{Au}-\mathrm{CdS}$ hybrid structure [395]. It is worth noting that the strong coordination bond between $\mathrm{Au}-\mathrm{S}$ is also conducive for the stabilization of Au at sulfur-rich end. Therefore, the interfacial energy that should be conquered between gold and cadmium-rich surface surpasses that of the gold and sulfur-rich surface, which play a dominant role in dictating the selective growth for many metal-chalcogenide heterostructures [391,396]. Interestingly, the role of anionic repulsive force could also denote a contribution to the growth of composite materials, which is seldomly considered. Recently, Yan's group [397] reported the facet-selective epitaxial growth of $\mathrm{NaYF}_{4}$ on $\mathrm{NaCl}$ nanocubes, which is a rare growth mode of traditional NCs. In their report, one-pot solution route method was used to produce the high quality $\mathrm{NaYF}_{4}-\mathrm{NaCl}$ core-cage NCs (Fig. 26). The repulsive force between $\mathrm{Cl}^{-}$and $\mathrm{F}^{-}$ions is the dominant role in dictating the facet-selective het-

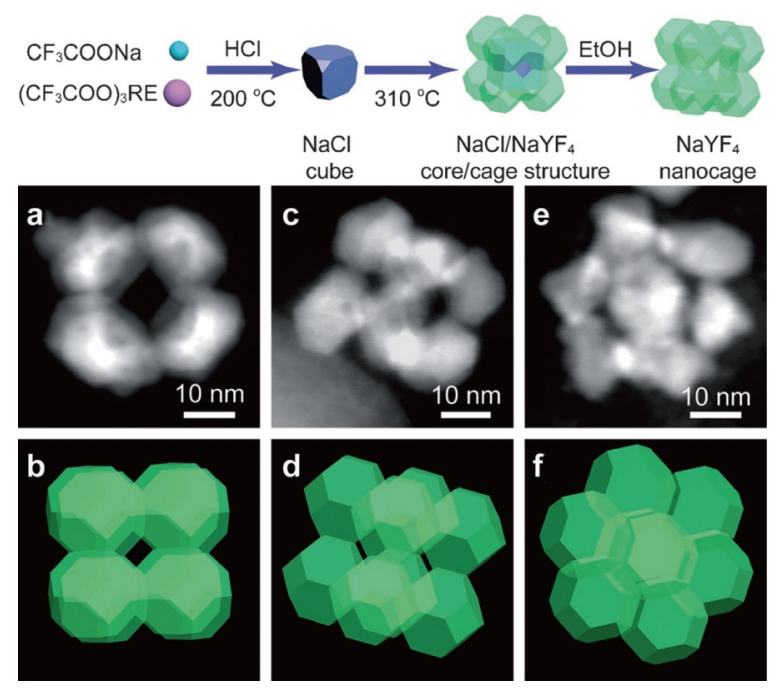

Figure 26 The top is the scheme for the formation of a $\mathrm{NaCl}$ cube, a $\mathrm{NaCl}-\mathrm{NaYF}_{4}$ core-cage structure, and a $\mathrm{NaYF}_{4}$ nanocage. HAADF-STEM images and corresponding models of an individual $\mathrm{NaYF}_{4}$ nanocage. (a, $\mathrm{c}$ and e) HAADF-STEM images observed in [001], [011], and [111] directions, respectively. (b, $\mathrm{d}$ and $\mathrm{f}$ ) Corresponding 3D models. Reproduced with permission from Ref. [397], Copyright 2012, WILEY-VCH Verlag $\mathrm{GmbH} \& \mathrm{Co}$. eroepitaxial growth of $\mathrm{NaYF}_{4}$ on $\mathrm{NaCl}$ nanocubes. From the viewpoint of crystallology, the $\mathrm{Na}^{+}$and $\mathrm{Y}^{3+}$ randomly occupy the face-centered cubic sites, while the $\mathrm{F}^{-}$locates at the tetrahedral sites, making the $\mathrm{NaYF}_{4} \mathrm{NCs}$ a calcium fluoride type structure. For $\mathrm{NaCl}, \mathrm{Na}^{+}$distributes at the face-centered cubic stacking sites and the $\mathrm{Cl}^{-}$takes all the octahedral sites, resulting in a similar fcc structure. The lattice mismatch between $\mathrm{NaYF}_{4}$ and $\mathrm{NaCl}$ can be calculated to $2.8 \%$ and both materials belong to the $F m 3 m$ space group. According to the DFT and the related experimental results, the epitaxial growth of $\mathrm{NaYF}_{4}$ on $\mathrm{NaCl}$ nanocubes is believed to be determined by the anionic repulsive force and the coordination numbers of $\mathrm{Y}^{3+}$. Their findings also open a new routine to control the heteroepitaxial growth by using the anionic repulsive force at the solid-solid interface.

\section{Cation exchange for heterostructures}

Cation exchange is an effective tool to introduce the complexity in synthesized NCs to form a multi-cation profile without tuning the synthetic parameters before the nucleation and growth. During the heterogeneous mechanism, the new phase nucleates at a surface facet and is followed by growth process, usually resulting in the phase topotaxially towards the interior of the NC. The modification in initial phase and the growth of new phase allow the use of partial exchange of interfaces or heterojunctions within a NC. For example, electron and hole transport and confinement for semiconductors can be systematically controlled by constructing the multicomponent NCs with heterojunctions or functional interfaces [398-401]. The precise control over the band engineering in heterostructures is highly desired for their optoelectronic applications such as light emitting diodes and photovoltaic cells.

Traditionally, producing a semiconductor onto seed NCs via the method of epitaxial growth is achieved by hot injection in multistep, however, the processing of cation exchange in the synthesis of semiconductor is simpler and more flexible. Taking $\mathrm{PbX}(\mathrm{X}=\mathrm{S}, \mathrm{Se}, \mathrm{Te})$ as an example, $\mathrm{PbSe} / \mathrm{CdSe}$ core-shell heterostructure has been obtained by the exchange of $\mathrm{Pb}^{2+}$ with $\mathrm{Cd}^{2+}$, where the exchange can be confined within the outermost shell of the NC $[402,403]$. More importantly, electron and hole carriers are confined within the lower band-gap PbSe core, largely improving the quantum yield excitonic emission and oxidative stability (Fig. 24a).

Manna' group [404] overcame the problems (uniform size, monodispersed shape, superior stability, the complex shape of these particles) and developed a new synthesis 
method to create octapod-shaped NCs, in which eight CdS "pods" were grown from a central CdSe NCs. In addition to the self-assembly of the highly oriented structures, they also found the $\mathrm{Cd}^{2+}$ ion can be exchanged by $\mathrm{Cu}$ in the $\mathrm{NC}$ by cation exchange followed by $\mathrm{O}_{2}$ plasma treatment. Although the exchange occurred in the solution quickly and almost completely, the 3D geometry of the assembly successfully was preserved. The as-formed $\mathrm{Cu}_{2} \mathrm{O}$ shell around each octapod will further enhance the mechanical strength.

Alivisatos and co-workers [405] demonstrated that in the synthesis of CdS- $\mathrm{Cu}_{2} \mathrm{~S} \mathrm{NC}$ heterostructures (Fig. 27a), the crystallographic selectivity for cation exchange to occur at different facets of ionic NCs played a critical role. Due to the high stability of $\mathrm{CdS}-\mathrm{Cu}_{2} \mathrm{~S}$ interfaces, the nucleation and growth of $\mathrm{Cu}_{2} \mathrm{~S}$ at the ends of CdS nanorods are preferential during the $\mathrm{Cu}^{+}$exchange formed at these facets. At the same time they found, there was nonselectivity in the synthesis of CdS- $\mathrm{Ag}_{2} \mathrm{~S} \mathrm{NC}$ heterostructures due to the nucleation in $\mathrm{Ag}^{+}$exchange which led to the formation of multiple $\mathrm{Ag}_{2} \mathrm{~S}$ regions within the nanorod. The differences between these two systems lie in both the chemical favorability for creating interfacial bonds and the elastic distortions between attachments connecting various facets of the two materials. The relative stabilities of the interfaces we have modeled correlate well with the frequency that the corresponding morphologies are observed. As both the shape and size of the NCs determine the crystallographic facets exposed at the surface, these parameters can be used to control the NC's reactivity. Recently, Zhang's group [406] prepared the $\mathrm{Cu}$-doped $\mathrm{CdS} \mathrm{NCs}$ from $\mathrm{Cu}_{2} \mathrm{~S}$ through a reverse cation exchange between $\mathrm{Cu}$ and $\mathrm{Cd}$. In detail, the cation exchange between $\mathrm{Cd}$ ions and $\mathrm{Cu}_{2} \mathrm{~S}$ NCs can indeed occur to obtain dominant $\mathrm{Cu}(\mathrm{I})$-doped CdS NCs. The atomic doping of $\mathrm{Cu}(\mathrm{I})$ can be verified by the X-ray absorption near edge structure (XANES) and extended X-ray absorption fine structure (EXAFS) spectroscopy [406]. Selective facet reactivity can in turn provide tunability of the physical properties of NC heterostructures through control of the spatial arrangement of their components. In their report, the cation exchange reaction, which is a useful chemical transformation tool, could overcome the limitation in traditional colloidal synthesis. In the cation exchange reaction, the atomic arrangement of the NC often follows the inherent symmetry of the underlying lattice. In their protocols used in cation exchange reactions, the used regents in the cation exchange with CdS, resulting in a highly anisotropic hybrid structure with $\mathrm{PbS}$ nanorods (Fig. 27b) [407], serve as an important probe for investigation of 2D

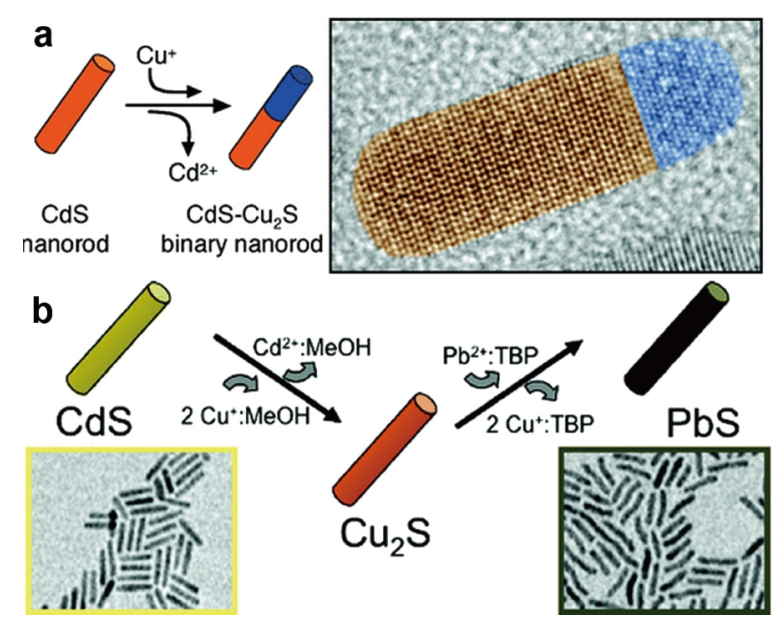

Figure 27 (a) The preparation of $\mathrm{CdS} / \mathrm{Cu}_{2} \mathrm{~S}$ hybrid structure. Reproduced with permission from Ref. [405], Copyright 2009. American Chemical Society. (b) The top is the preparation of PbS nanorods. Reproduced with permission from Ref. [407], Copyright 2009, American Chemical Society.

quantum confinement. If the hetero NCs are composed of different segments, the composition and the growth direction of the foreign part derived from the cation exchange reaction can be highly selective for the heterostructure.

$\mathrm{Kim}$ and the co-workers [408] reported the $\mathrm{CuInS}_{2} / \mathrm{ZnS}$ core/shell quantum dots by cation exchange. In their report, the $\mathrm{CuInS}_{2}$ quantum dots were prepared firstly, followed by the cation exchange reaction by $\mathrm{Zn}^{2+}$ to finally generate the $\mathrm{CuInS}_{2} / \mathrm{ZnS}$ core/shell quantum dots. The core/shell quantum dots were confirmed by TEM, which indicated the cation exchange reaction occurred in the internal core. So, the increased blue-shift in their emission was believed to be ascribed to the reduced core size. Also, they extended this partly cation exchange to prepare the $\mathrm{AgInS}_{2} / \mathrm{ZnS}$ core/shell quantum dots, which showed a blue shift of $70 \mathrm{~nm}$ in the emission spectra [409]. Agarwal and co-workers [410] used the protocol of size-dependent cation exchange reactions in transformation of composition-controlled single-crystalline nanowires involving the cation exchange between $\mathrm{Zn}$ and $\mathrm{Cd}$. As a result, a series of $\mathrm{NC}$ heterostructures such as $\mathrm{Zn}_{x} \mathrm{Cd}_{(1-x)} \mathrm{S}$ metal-semiconductor superlattices $\left(\mathrm{Zn}_{x} \mathrm{Cd}_{(1-x)} S\right)$, single-crystalline $\mathrm{ZnS}$ nanotubes, and eventually metallic $\mathrm{Zn}$ nanowires can be successfully prepared by a sequential strategies including size-dependent cation-exchange reaction, temperature control and gas-phase reactant supply. In their report, the thin nanowire $(<25 \mathrm{~nm})$ of CdS could be transformed into $\mathrm{ZnS}$ nanowire completely. Intermediate sized nanowires (30-90 nm) undergo radially uniform partial cation exchange and their bandgaps shift to higher energies with 
increasing $\mathrm{Zn}$ content, which was confirmed by photoluminescence measurements. Thicker nanowires $(>120 \mathrm{~nm})$ were obtained by selective cation exchange localized to a region of $25-30 \mathrm{~nm}$ from the surface, leading to formation of core -shell heterostructures with CdS and $\mathrm{Zn}_{x} \mathrm{Cd}_{(1-x)} \mathrm{S}$ in the core and the shell regions.

\section{Exfoliation}

Since Novoselov et al. [412] reported a few layer graphene films can be prepared by mechanical exfoliation (repeated peeling) from pyrolytic graphite, exfoliation has been confirmed to be an important strategy to obtain single layer or a few layer 2D structure for layered materials. The as-prepared graphene, which is a few atoms thick, exhibits monocrystalline fashion and strong ambipolar electric field effect. This top-down method ensures the successful preparation of graphene thin film up to $10 \mu \mathrm{m}$ in size. To satisfy the demand of exploiting the properties of graphene, large scale growth and large-scale exfoliation methods have been reported. The pristine method for preparing graphene is the "peeling off" [413] and "epitaxial growth" [414,415]. For the top-down method in solution system, Li et al. [411] reported that the exfoliation-reintercalation-expansion of graphite can generate single-layer graphene sheets with high quality which can disperse in organic solvent. They used the expandable graphite, which was obtained by chemical interaction of sulphuric acid and nitric acid [416], as starting precursor to prepare single-layer graphene. This high-quality single-layer graphene can readily turn into large transparent conducting films in a layer-by-layer manner through Langmuir-Blodgett assembly (Fig. 28). This synthetic strategy shed light to the chemically derived manufacturing of scalable graphene devices. Coleman and co-workers [417] demonstrated the organic solvents such as $\mathrm{N}$-methyl-pyrrolidone can result in the exfoliation of graphite and generation of graphene solution with concentrations up to $\sim 0.01 \mathrm{mg} \mathrm{mL}^{-1}$. The increased surface energy of each single-layer graphene is believed to be balanced by solvent-graphene interaction whose energies can match the exfoliation. Based on this method, individual graphene can be prepared, which was evidenced by Raman spectroscopy, TEM and electron diffraction. According to the X-ray photoelectron, infrared and Raman spectroscopies, the as-prepared graphene is defect-free and graphene oxide-free. These findings indeed enable a range of potential large-area application of graphene through the large-scale synthesis.

Later, a series of $2 \mathrm{D}$ layered materials such as $\mathrm{MoS}_{2}, \mathrm{WS}_{2}$, $\mathrm{MoSe}_{2}, \mathrm{MoTe}_{2}, \mathrm{TaSe}_{2}, \mathrm{NbSe}_{2}, \mathrm{NiTe}_{2}, \mathrm{BN}$, and $\mathrm{Bi}_{2} \mathrm{Te}_{3}$ nano-

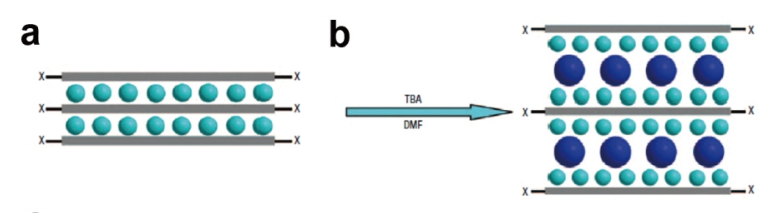

C
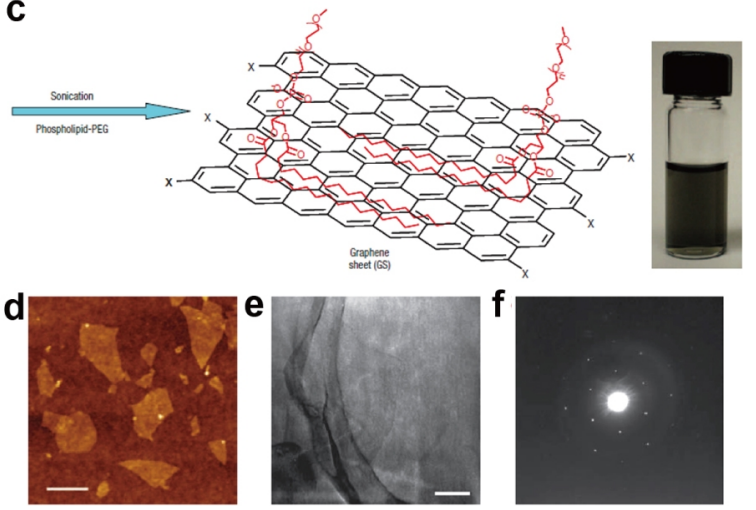

Figure 28 (a) Schematic of the exfoliated graphite reintercalated with sulphuric acid molecules (teal spheres) between the layers. (b) Schematic of tetrabutylammonium (blue spheres) insertion into the intercalated graphite. (c) Schematic of graphenen sheets (GS) coated with distearoyl-sn-glycero-3-phosphoethanolamine conjugated polyethylene glycol (DSPE-mPEG) molecules and a photograph of a DSPE-mPEG/dimethylformamide (DMF) solution of GS. (d) An AFM image of a typical GS. The scale bar is $300 \mathrm{~nm}$. (e) Low-magnification TEM images of a typical GS. The scale bar is $100 \mathrm{~nm}$. (f) Electron diffraction (ED) pattern of an as-made GS. Reproduced with permission from Ref. [411], Copyright 2008, Nature Publishing Group.

sheets have been produced by liquid exfoliation, which can generate thin films with a few layers thickness [419]. This discovery predicts that the layered bulk materials can serve as the diverse source to obtain $2 \mathrm{D}$ crystals which exhibit useful application in the areas of electronics, optics and energy storage. Electron microscopy results clearly indicate that the layered materials can be exfoliated into individual layers which can be deposited as individual flakes or form into films. Ramaprabhu and co-workers [420] extended the exfoliation of graphite oxide by using focused solar radiation as driving force for highly conducting graphene. Zhang's group [50,418,421] developed an electrochemical Li-intercalation and exfoliation method which was effective to prepare various metal dichalcogenide, $\mathrm{BN}$ and graphene nanosheets. A Li-ion battery setup with the layered bulk crystal as cathode and lithium foil as anode was used to exfoliate the layered materials (Fig. 29). After the lithium intercalation by discharge process, the Li-intercalated layered structures $\left(\mathrm{Li}_{x} \mathrm{MoS}_{2}\right.$, where $x$ is the number of $\mathrm{Li}$ atoms in $\mathrm{Li}_{x} \mathrm{MoS}_{2}$ ) will form. During the sonication in water, the intercalated Li will react with water and generate $\mathrm{H}_{2}$, which can exfoliate the 


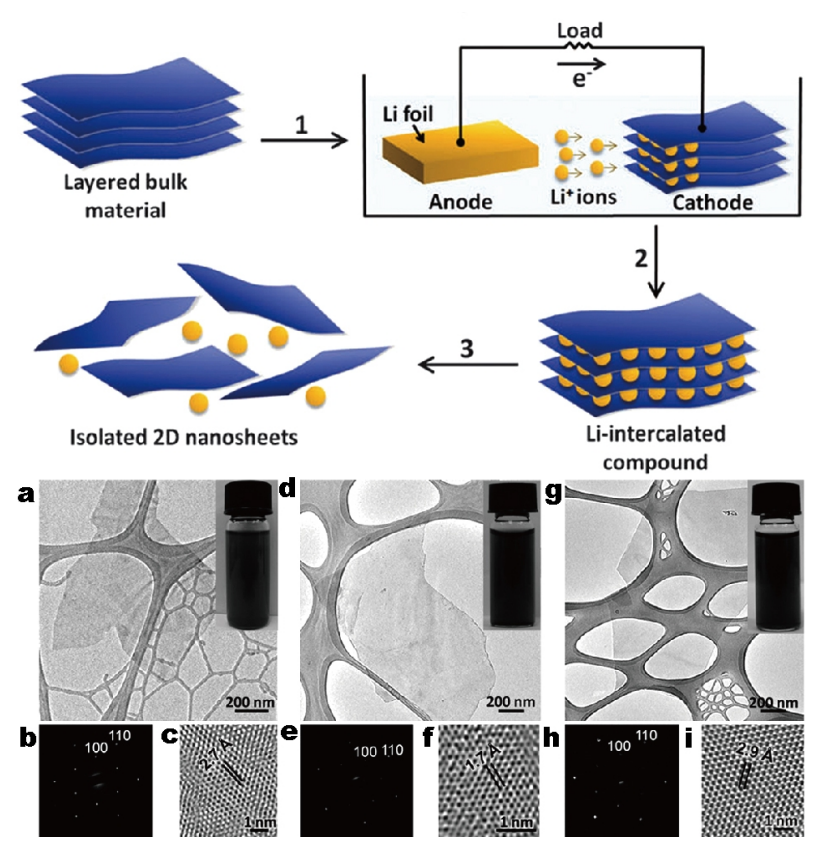

Figure 29 The top is the scheme of electrochemical lithiation and exfoliation process for fabrication of 2D nanomaterials from the layered bulk crystals. (a) TEM image of a typical single-layer $\mathrm{WS}_{2}$ nanosheet. (b) Selected area electron diffraction (SAED) pattern of a single-layer $\mathrm{WS}_{2}$ nanosheet. (c) HRTEM image of a single-layer $\mathrm{WS}_{2}$ nanosheet. (d-f) TEM image, SAED pattern and HRTEM image of a single-layer $\mathrm{TiS}_{2}$ nanosheet. (g-i) TEM image, SAED pattern and HRTEM image of a single-layer $\mathrm{TaS}_{2}$ nanosheet. Reproduced with permission from Ref. [418], Copyright 2011, WILEY-VCH Verlag GmbH \& Co.

layered structure into one-layer nanosheets. This method offers an excellent opportunity to control the Li-intercalation by varying the discharge curve, which can optimize the quantity of Li and quality of the final products.

Exfoliation is an interesting route for producing LDH nanosheets. Unlike the cationic clays such as montmorillonite and laponite that can be exfoliated into single clay sheets, the exfoliation of LDHs into charged thin platelets is difficult in aqueous solution. The strong interlayer electrostatic interactions between the LDHs layers with high charge density and the high anion content result in the difficulty to be delaminated. Further, the lamellaes will stack tightly due to the extensive interlamellar hydrogen bonding networks. A good solution is combining the charged thin platelets with a thickness of a few atomic layers with polymers or other building units to form designed organic-inorganic or inorganic-inorganic nanocomposites. The first example of delamination of $\mathrm{Zn}-\mathrm{Al}-\mathrm{NO}_{3} \mathrm{LDHs}$ was achieved by using sodium dodecylsulfate as an anionic surfactant and butanol as the dispersant, which was reported by Adachi-Pagano and co-workers in 1999 [422]. Later, O'Leary et al. [423] reported the delamination of LDHs in acrylates. Afterwards, Jobbágy and Regazzoni [424] extended the exfoliation systems to the solvents of toluene and $\mathrm{CCl}_{4}$. After a few years of development about the synthetic methodology on LDHs and its exfoliation, Ma et al. [425] contributed a brief review on how to delaminate the LDHs and gain the corresponding positively charged nanosheets in formamide. In detail, LDHs with nitrate as counter anions can be exfoliated into high-yield, micrometer-sized and well-defined LDH nanosheets [426,427]. An LDH-carbonate was firstly prepared via the so-called homogeneous precipitation. Then, a conversion into a nitrate form was observed through a unique acid-salt exchange procedure. Finally, the complete exfoliation was finished by the intercalation of formamide.

\section{BOTTOM-UP AND BOTTOM-UP}

In the catalytic field, interfacial behaviors have been proven to be where the reaction takes place. Actually, the interfaces resulted from the foreign species may enhance the activity, selectivity and durability. For example, the interface between Au and Pt can indeed strength the preferential oxidation of $\mathrm{CO}$ and facilitate the resistance against dissolution of Pt under potential cycling regimes [428]. In addition, designed interface between metal and metal-organic frameworks (MOFs) was also built up by sequential nucleation to achieve gas harvest and molecular sieving $[150,429,430]$. Bao and co-workers [431] reported the interface between metal and oxides can be utilized to stabilize the coordinatively unsaturated ferrous sites, which could largely improve the catalytic performance towards reactions involving dioxygen activation such as $\mathrm{CO}$ oxidation at low temperature. Most recently, Zheng's group [432] reported the fabrication of interface between the surface capping agents and metal could impart the functionality to tune the electronic structure of the metal catalysts, which could further endow the control over the selectivity during the catalytic performance. Since the interfacial structures play a pivotal role in modulating the intrinsic properties such as catalytic behavior, considerable research efforts have been made for developing the strategy to effectively fabricate interface between metal-metal, metal-oxides, metal-semiconductor, metal-organic and so on. In this review, we focus on the typical procedures and recent advances for optimizing the synthetic strategy over the composition, shape, interface and structures especially the composites and complex structures. In each section, the important and representative works are given to illustrate how to use this strategy to achieve the tailoring of complex and hybrid architectures. 


\section{Seed growth}

As each reaction stage brings along adjustable experimental conditions, such as temperature and pressure, constructing multiple stages in producing a functional nanomaterial enables more free parameters, which can result in more flexibility and precision of tailoring the functionality of the final product. The seed-mediated growth approach is an extremely popular method in fabricating metal [433-435], semiconductor $[67,436-438]$ and meal oxide $[40,439]$ NPs with complex structure and novel functions. Naturally, the nucleation and growth can be regarded as two different stages since they undergo different chemical processes [41]. The nucleation process requires supersaturation of the solute (for example, zero-valent atoms), which is sometimes referred as critical concentration. In this stage, the minimization of Gibbs free energy drives the formation of the seed [90]. In the growth stage, these pre-obtained seeds are used as heterogeneous nucleation sites to deposit other materials. The activation energy for the secondary material deposition onto an already formed particle is significantly lower than for homogeneous nucleation of seed particles in solution, thus milder experimental condition such as lower temperature can be used [41]. Tuning the size, shape, structure, and composition of both the seed and overgrowth material enables the flexibility of designing the properties of nanomaterials.

The simplest case of seed-mediated growth is to grow the same material on the surface of the seed. A classic example is seed-mediated synthesis of gold nanorods $[68,91,98,123]$. In chemical synthesis, gold nanorods are produced by reducing gold ions in the present of small nanogold seed [97]. Using the seed-mediated method, the aspect ratio of the gold nanorods, which is crucial for the plasmonic mode within the rods, is adjustable by changing the experimental conditions such as the amount of the reduction agent or $\mathrm{Ag}^{+}$ions in the solution in the growth stage $[3,8,37,68,440]$ (Fig. 30). This example demonstrates the flexibility of controlling the shape of the final product using seed-mediated growth. In fact, the parameter of the seeds, for example, the structure, is equally important for the shape of the final product $[87,433]$. In the community of shape-controlled synthesis of noble metal NPs, such as Ag [131], Pt [42] and Pd [87], selective adsorbates are frequently used to block the growth of certain facet ( $\{111\}$ or $\{100\})$ of the NPs [433], thus the final product exposes particular facets. As a consequence, the shape of the NPs can be controlled. However, different initial seed structures can result in the variation of the final shape of the NPs despite the same type of adsorbate is used (Fig. 31). If the seed starts from a single cry-

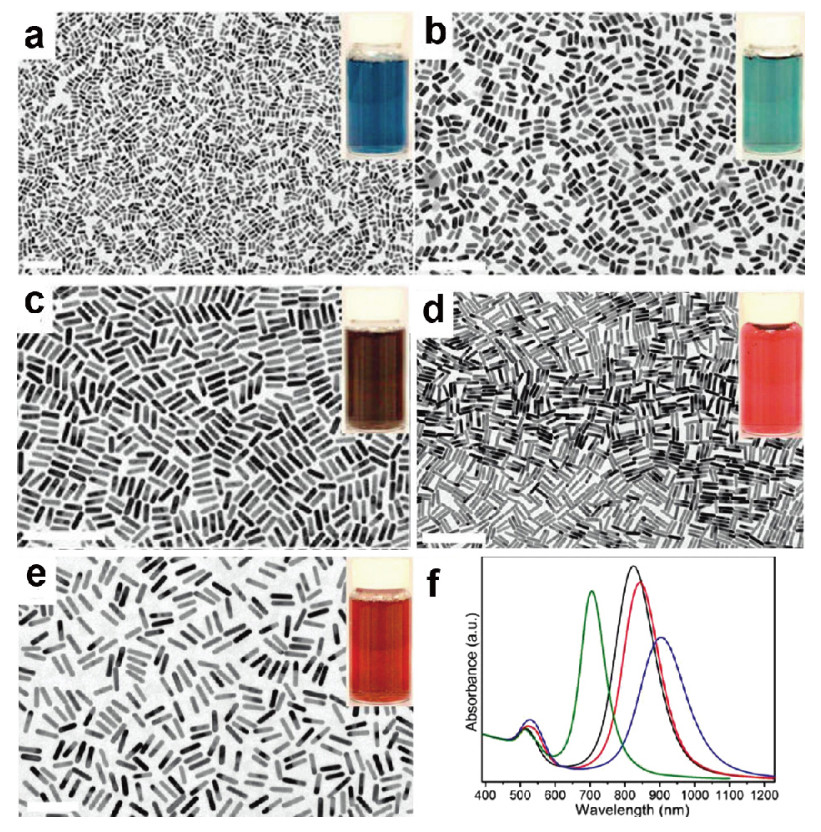

Figure 30 (a-e) The TEM images and (f) corresponding UV-vis-NIR spectra of Au nanorods with different sizes. Reproduced with permission from Ref. [440], Copyright 2012, American Chemical Society.

stal seed, the shape-controlled NPs usually end up with cube (with $\{100\}$ facet adsorbate), bar (with $\{100\}$ facet adsorbate), octahedron (with $\{111\}$ facet adsorbate) or tetrahedron (with $\{111\}$ facet adsorbate). If it starts from lamellar twinned NPs, bipyramid (with $\{100\}$ facets adsorbate) or triangle or hexagonal plate (with $\{111\}$ facet adsorbate) shape can be obtained. In the case of multiply twined seeds, the final product is rod with fivefold symmetry (with $\{100\}$ facet adsorbate) or quasi-spherical decahedral or icosahedral NPs (with $\{111\}$ facet adsorbate).

When another material is deposited on the surface of the seed, the growth mode of the secondary materials is crucial to the property of the final product. There are two typical growth modes for the growth of the secondary material, Frank-van der Merwe (F-W) mode and Volmer-Webber (V-W) mode (Fig. 32). Stranski-Krastanow (S-K) is the hybrid of F-W and V-W modes. In the case of F-W growth mode (epitaxial growth), the secondary material grows on the surface layer by layer. V-W means the secondary material grows on the surface island by island.

The growth mode is determined by the physical parameters such as lattice mismatch, difference in electronegativity, interface energies, and diffusion rate. Lattice mismatch is a measure that quantifies the difference in lattice plane,

$$
L=\left|\frac{a_{1}-a_{2}}{a_{1}}\right|,
$$




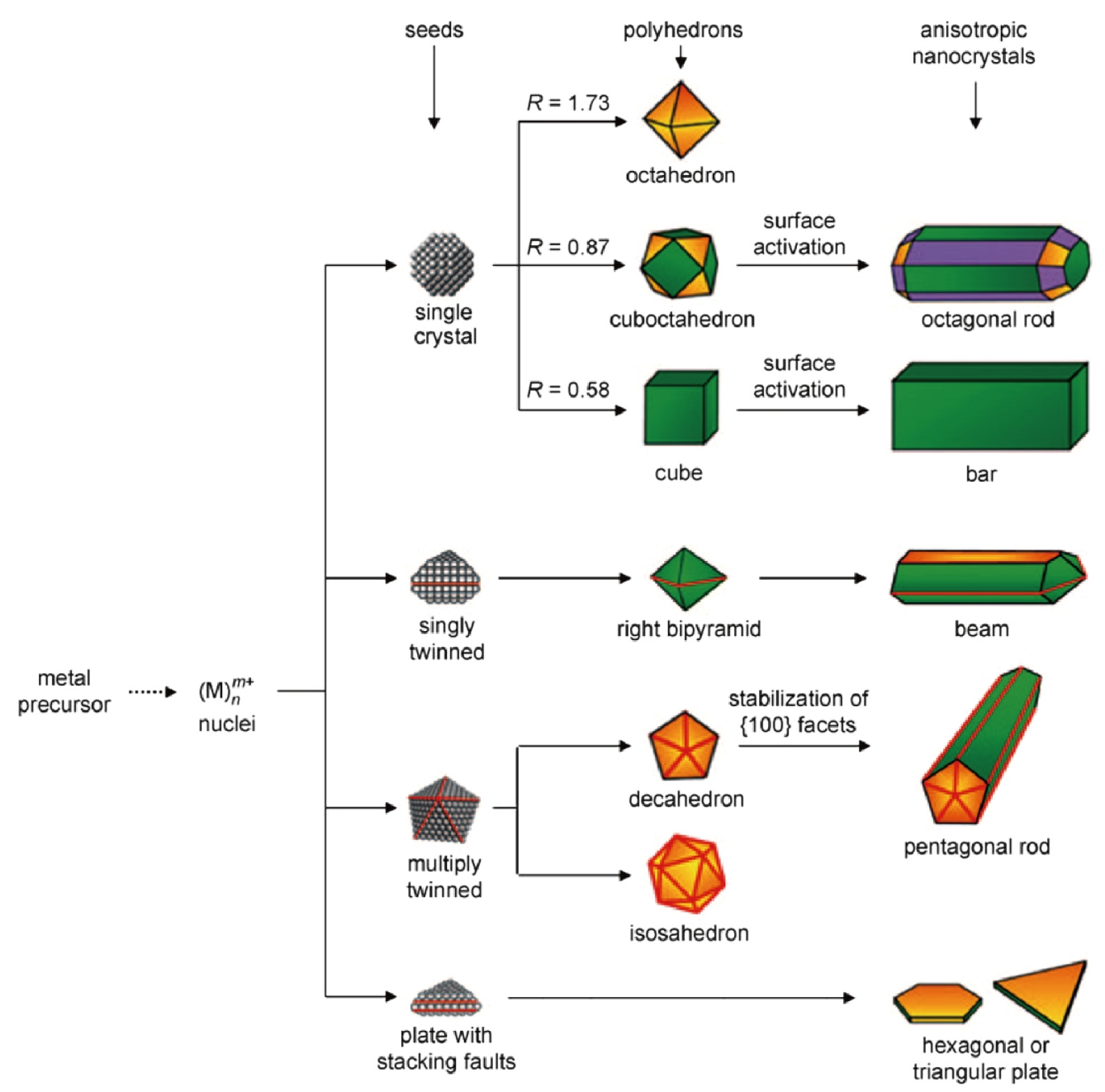

Figure 31 Reaction pathways that lead to fcc metal NCs having different shapes. Reproduced with permission from Ref. [2], Copyright 2009, WILEYVCH Verlag GmbH \& Co.

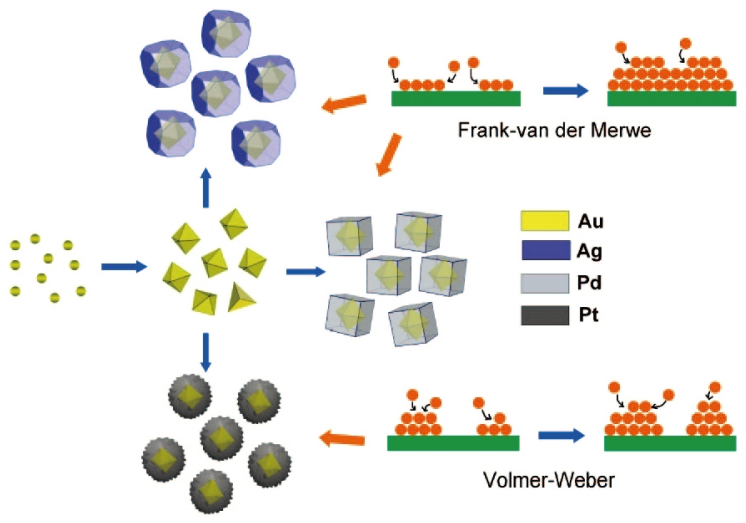

Figure 32 Illustrations of the growth model on the surface of a seed. Reproduced with permission from Ref. [225], Copyright 2008, American Chemical Society. where $a_{1}$ and $a_{2}$ are the lattice parameters of growing planes for the seed and the overgrowth material, respectively. A lattice mismatch can cause strain within the two materials. To grow the material on a surface with less defects, an epitaxial growth mode is usually preferred. Epitaxial growth requires close lattice match between the seed and overgrowth materials (less than 5\%), for example Pd and Pt [95]. If the lattice match is large enough, the lattice strain is so large that the overgrowth layer cannot form complete layer with thickness more than the critical layer thickness (about two monolayers). Therefore, the epitaxial condition is broken and the overgrowth material forms island on the seed with random orientation (V-W mode). This nonepitaxial growth prevents the conformal overgrowth and generates defects which can lead to the failure of functional 
nanomaterials. For example, owning to the lattice strain, it is very difficult to grow $\mathrm{CdS}$ or $\mathrm{ZnS}$ on the surface of CdSe, despite the coating can greatly improve the optical quality [146]. To overcome the lattice mismatch limit for epitaxial growth, Gu et al. [85] found shrinking the size of Pd seed down to tens of nanometers can successfully grow $\mathrm{Cu}$ epitaxially on the surface despite their large lattice mismatch is $\sim 7.1 \%$. Xiong et al. [77] reported the nonepitaxial growth of coating single crystalline CdS on Au core with large lattice mismatches. Through cation exchange, it is possible to trigger the amorphous AgS layer to transform into a single crystalline layer CdS. The electronegativity is of importance in the case of depositing a metal on another metal. If the electronegativity of the seed is lower than the secondary metal, it will cause the galvanic replacement, instead of the epitaxial growth, between the core and the shell. This phenomenon has been observed in the system of Pt@Au,Ag@Pd,Ag@Au and so on [81]. In addition, to grow in a layer-by-layer fashion, the bonding between the seed and the secondary material should be stronger than the bonding within the overlayer. Otherwise the secondary materials tend to nucleate within themselves, such as in the system of Pt on the surface of $\mathrm{Au}$ [153].

The diffusion of atoms is also a possible parameter that can affect the final product synthesized by seed-mediated growth. The diffusion rate can be written as an Arrheniuslike equation as follows,

$$
D=D_{0} \exp \left(-E_{\text {diff }} / R T\right),
$$

where $D_{0}, E_{\text {diff, }} R$ and $T$ are the diffusion preexponential factor, the potential energy barrier to diffusion, the ideal gas constant, and the absolute temperature (in Kelvin), respectively [130]. There are two types of diffusion, lateral diffusion and inter diffusion. Lateral diffusion is the diffusion of the secondary material on the surface of the seed. In general, higher temperature can promote the atom diffusion on the surface. Also, the size of the seed, with respect to the diffusion distance, can have impact on the morphology of the product. It has been investigated by Zhuang et al. [130] on both Pd-Pd and Pd-Pt systems, where adatoms can reach to the side faces much faster when the seed is smaller. The inter-diffusion between the seed and the secondary material can also be used as a tool to construct the structure and shape of the final product. For example, Wang et al. [145] showed that in the Pd-Pt core-shell NPs produced by the seed-mediated growth method, Pd atoms can diffuse into the Pt layer, which formed Pd channels in the Pt layer. These channels are extremely crucial for the synthesis of
Pt-enriched cages through selective removal of Pd. In addition, by employing this inter-diffusion mechanism, more complicated NPs can be synthesized. For example, if the outward diffusion rate is much faster than the inward diffusion of the overgrowth materials, the NPs could become a hollow structure, i.e., Kirkendall effect $[48,125]$.

The most important application of seed-mediated growth is probably to construct core-shell NPs, for example, constructing Pt-based core-shell NPs. As the Pt is a very expensive material and extremely rare in the earth's crust, Pt-based core-shell structure recently draws attention as it has the advantage of replacing the core with cheaper and more abundant materials, such as Pd. The sophistication in shape-controlling of Pd seed and its moderate lattice mismatch give more freedom in designing the exposed facet and structure of core-shell Pd-Pt NPs, such as cube [156], octahedron [154] or icosahedron [155]. Also, for materials that is extremely easy to homogenously nucleate, such as Ir, which usually results in roughly spherical shape, seed-mediated growth offers a possible way to control its shape [441]. In addition, the seed-mediated growth method has also been combined with other techniques such as lithography [40]. For example, by depositing the $\mathrm{ZnO}$ seeds over a mask with a particular pattern, the further overgrowth of $\mathrm{ZnO}$ can maintain the pattern and position on the substrate. Eustis and El-Sayed [71] demonstrated a general approach to fabricate the $\mathrm{ZnO}$ on a universal substrate, which enabled this technique to find potential applications in nanodevices.

\section{Self-assembly}

Self-assembly is a common phenomenon in material area and even the nature. The reproducible formation of a hierarchical structure of myriad possibilities requires further information such as instructions or guidance to dictate the programmed assembly. The information added to the assembly must contain the location and connectivity of the building blocks within the assembled structure. As a sequential bottom-up approach, the information is mainly distributed among the building blocks and the corresponding interactions executed by spontaneous process under physical and/or chemical driving force [442]. Accounting for the interaction that determines the system's free energy and constraints that determine the accessible configurations, the encoded information plays a decisive role in the complexity of the final structure under equilibrium. In detail, the colloidal interactions usually include van der Waals, electrostatic, magnetic, molecular surface forces, depletion and DNA hybridization and so on, which can 
largely promote the designing of various monodispersed particles with well-defined sizes and shapes [443-445]. Recently, the by-product of $t$-butylamine hydrochloride generated from the reduction of metal precursor was found to facilitate the self-assembly of Au superlattice [446]. Most recently, Wang et al. [447] denoted a comprehensive review of understanding the forces involved in the self-assembly of 3D superlattice.

The self-assembly of colloidal NCs is a promising method to fabricate highly ordered nanostructures, which may enhance mechanical, electronic and magnetic properties $[448,449]$. These improvements can promote the corresponding applications such as energy capture and storage, theranostics (combined diagnostics and therapy), photonics and electronics. In 1995, Murray and co-workers $[312,450]$ demonstrated the self-organization of monodispersed CdSe NCs into 3D superlattices. During the assembly, the size and spacing of the quantum dots were well controlled with atomic precision. Actually, this work shed light to the fast development of self-assembly in the following years. The assembly routes for monodispersed NCs of insulating, semiconducting, magnetic, oxides materials are readily achieved by other groups. Redl et al. [451] extended the self-assembly to a binary NP superlattices. They selected the monodispersed PbSe semiconductor quantum dots and $\mathrm{Fe}_{2} \mathrm{O}_{3}$ magnetic NCs as the building blocks to fabricate well-defined ordered $3 \mathrm{D}$ superlattices through self-assembly. The different specific size of these two types of NCs make the final structures into $A_{13}$ or $A B_{2}$ superlattices whose optical and magnetic properties can be further tuned by the control over the assembly process. These binary NCs system has been further studied by Murray's group. In detail, 15 different binary NP superlattice structures were further discovered and investigated by using different combinations of semiconducting, metallic and magnetic NPs building blocks [452]. Afterwards, the same group discovered the quasicrystalline order in self-assembled binary NP superlattices [453]. They observed that the formation of assemblies with dodecagonal quasicrystalline order during the self-assembly process can be archieved by several combinations of two different NCs: 13.4-nm $\mathrm{Fe}_{2} \mathrm{O}_{3}$ and 5-nm Au NCs, 12.6-nm $\mathrm{Fe}_{3} \mathrm{O}_{4}$ and 4.7-nm Au NCs, and 9-nm PbS and 3-nm Pd NCs. Such compositional diversity reveals the formation of quasicrystalline-like assemblies is a common phenomenon in nature, which is believed to attribute to the driving force of entropy and strong interparticle interaction. Our group discovered a simple and facile microemulsion-based approach for the synthesis of a wide variety of colloidal spheres with different sizes, shapes, and properties by using dispersible NCs [454]. Moreover, other types of NPs such as semiconducting metal chalcogenides, metal oxides, and rare-earth compounds with the hydrophobic ligands on the surface can serve as the building blocks by taking advantage of the oil-in-water $(\mathrm{O} / \mathrm{W})$ microemulsion system. The hydrophobic van der Waals interaction between the surfactant ligands will gather, assemble and fix the NCs together spontaneously. After the evaporation of the low-boiling oil solvent, a restricted, micrometer-sized 3D colloidal sphere will form from the microemulsion droplets. Our discovery enables the preparation of new types of 3D colloid crystals from monodispersed NCs. By the way, the hydrophobic building blocks will be changed to water-soluble after the self-assembly process due to the usage of hydrophilic surfactants. The oil phases play an important role in the formation of colloidal sphere: (1) the low-boiling solvent in the oil phase can be easily evaporated; (2) the nonpolar oil media can serve as excellent solvents to disperse the building blocks capped with hydrophobic ligands. The basic processes and principles of the emulsion-based bottom-up self-assembly method are illustrated in Fig. 33. Using one single building units such as $2-\mathrm{nm}$ Au NPs, multivalent assembly to generate one-, two-, and three-dimensional nanostructures can be constructed by kinetically controlling the diffusion of ultrasmall Au NPs [455].

The addition of small adsorbates such as metal ions, polymer, and amino acid can also result in the nanoassemblies based on the strong interaction with the NPs' surface. Hong et al. [456] presented the addtion of Pluronic P123 surfactant can lead to the assembly of AuAg nanowires into the AuAg nanobelts. Sun et al. [457] discovered the usage of $\mathrm{Fe}$ ions into the synthesis of Pt nanocubes can dictate the formation of $\mathrm{Pt}$ nanocube assemblies. This process may result from the interparticle electrostatic repulsion between charged-NPs [458,459]. Chen's group [460] showed the arginie, which is an amino acids owning guaidino group, can be utilized to interact with carboxyl groups on Pt NPs' surface by hydrogen-bond and electrostatic interaction to lead to the self-assembly of Pt NPs.

In addition to the spherical nanoscale building blocks, significant interest has been generated to the anisotropic 1D NCs and 2D nanosheets $[456,461,462]$. Zhang et al. [463] contributed a review about the self-assembly of colloidal 1D NCs. Especially, the attention was paid to the anisotropic nanowires and nanorods and several strategies were highlighted in the review. In summary, the self-assembly of $1 \mathrm{D}$ NCs usually takes place under the following 

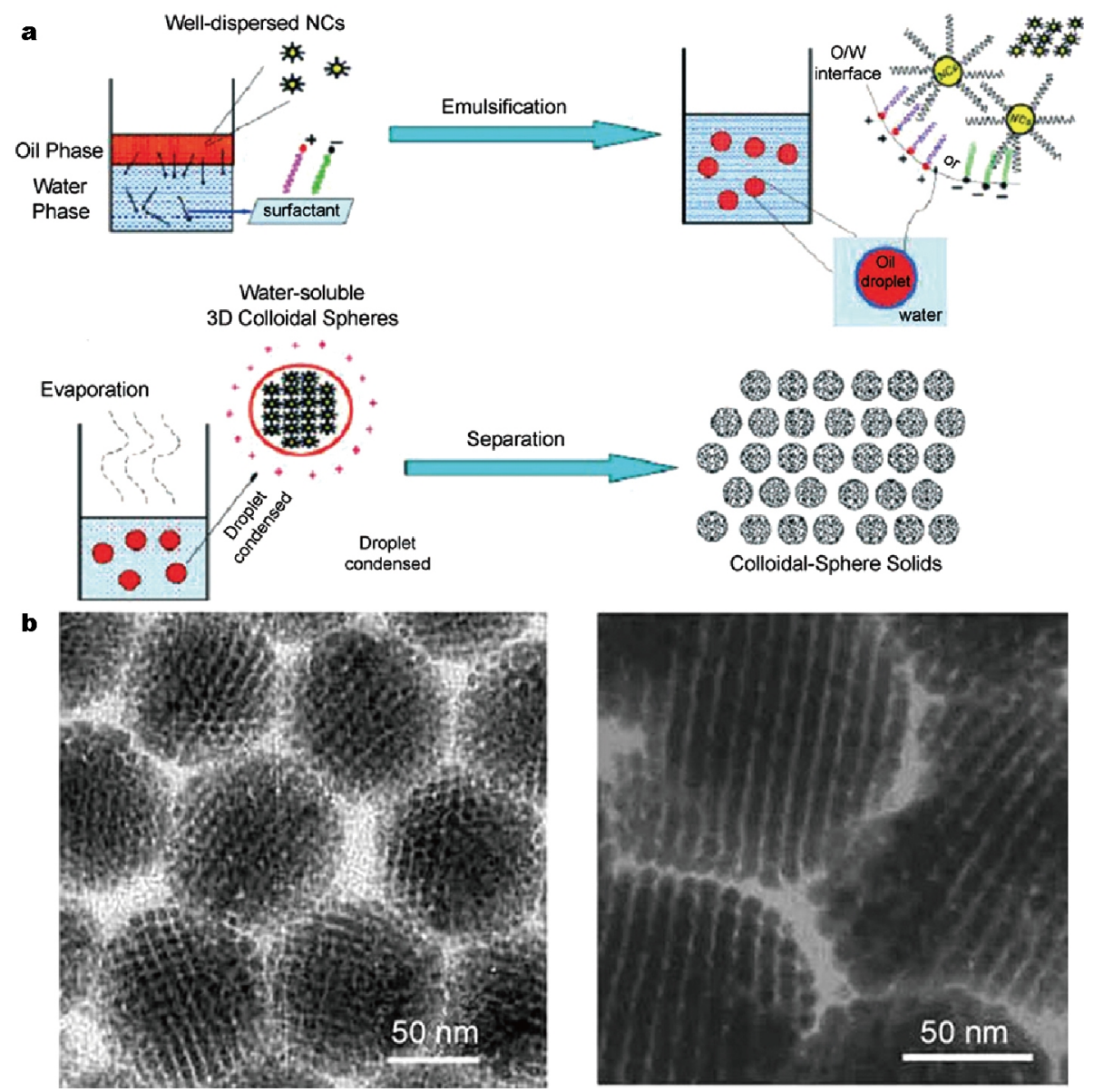

Figure 33 (a) Schematic of the emulsion-based bottom-up self-assembly method. (b) Typical TEM images and high-resolution images of $\mathrm{BaCrO}_{4}$ colloidal spheres. Reproduced with permission from Ref. [454], Copyright 2007, WILEY-VCH Verlag GmbH \& Co.

situations: (1) substrates driven by evaporation under external field facilitation or template, (2) interface of liquid-liquid and gas-liquid, and (3) solution through specific chemical bond. For example, Wang et al. [464] reported the construction of self-assembly from the complex building blocks of CdSe-CdS core-shell nanorods. Their strategy involves the generation of water-dispersible nanorod micelles in mixing a chloroform solution and evaporation of chloroform (Fig. 34). TEM tilting experiments demonstrate that the resulting structure is made up of multiple well-defined supercrystalline domains which are strictly dependent on the total number $(N)$ of constituent nanorods in each assembly structure.

Despite fruitful progress towards "programmability" has been made in the past decades, self-assembly remains limited to highly symmetric structures such as nanowire, nanoplate, small clusters, polyhedron, superlattice and linear chains [465-475]. It is still challenging to extend the self-assembly to arbitary structures with specified size and defined building blocks. In a recent commentary, Bishop and Cademartiri [476] proposed two conceptual strategies of puzzle and folding approaches which may guarantee the equilibrium self-assembly of colloidal building blocks into arbitrary structures. Looking forward, how to achieve the "complex matter" which is found in natural materials such as muscle, wood or the cytoskeleton may require the encoding information within the building blocks.

The arrangement of colloidal NC into highly ordered architecture is a first step to simulate the fabrication of artificial solids and new devices. As is well known, several types of forces were believed to be involved in the self-assembly process such as van der Waals attractions between the particles, steric repulsions between the long-chain surfactants, capillary forces during solvent evaporation, attractive depletion forces, Coulombic repulsive forces between two positive surface charges or two electric dipoles, and magnetic forces [443,445,477-480]. In most cases, for example, the assembly by spherical NPs, smectic-like multilayers of 


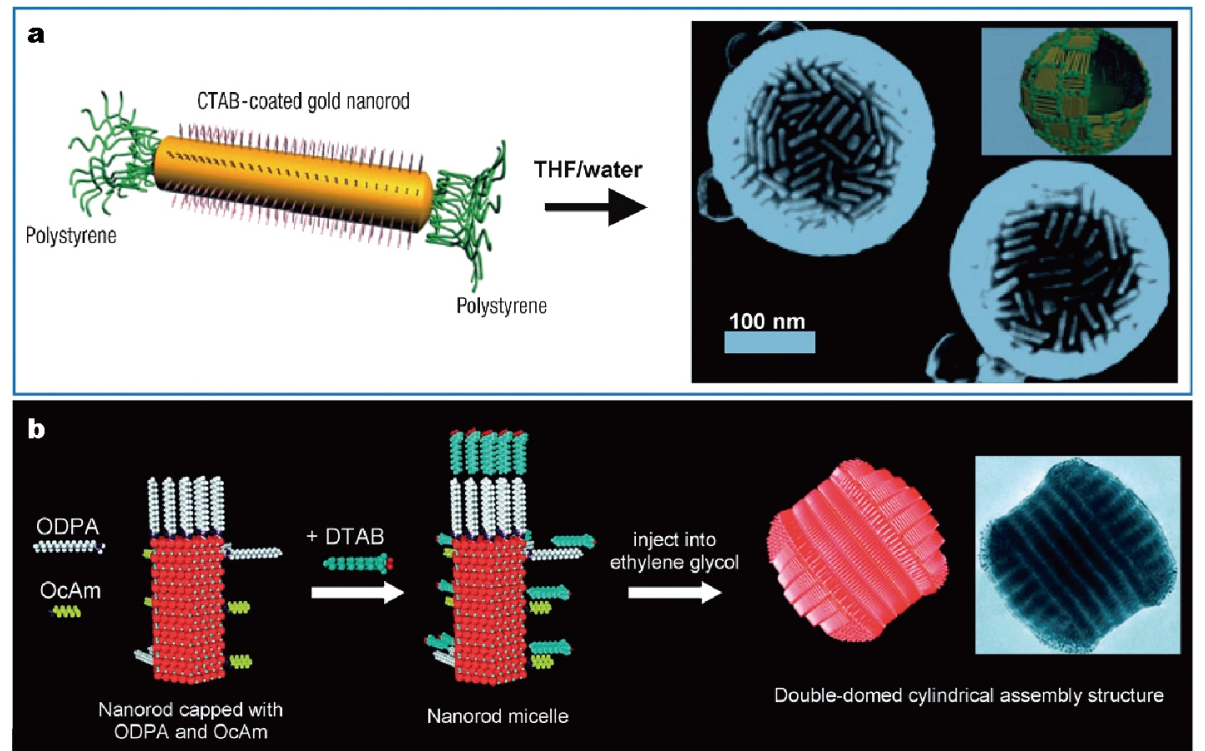

Figure 34 (a) Schematic illustration that shows the formation of 3D hollow spherical assembly structures from amphiphilic Au nanorods. SEM images of the self-assembled structures are also shown. (b) Schematic depiction of the formation of a 3D double-domed cylindrical structure from core-shell CdSe-CdS nanorods capped with octadecylphosphonic acid and octylamine. Also shown is a representative TEM image of the resulting $3 \mathrm{D}$ assembly structure. Reproduced with permission from Ref. [464], Copyright 2012, the American Association for the Advancement of Science.

hexagonally packed nanorods and so on, the assembly was found to be solely driven by entropy [481-483]. The combination of the nanometer-scale building blocks and their synergistic effect endows these assemblies novel properties for their further applications in thermoelectric, photovoltaic, memory storage, catalysis, and chemical sensing aspects [484,485]. In 2011, Manna’s group [486] demonstrated linear chains of interlocked octapods with two sequential levels can be self-assembled by monodispersed colloidal octapod-shaped NCs self-assemble. Moreover, the mechanical strength of these architectures was significantly improved by welding the unit cell together. The approach they presented such as cation exchange during the self-assembly showed an innovative route towards the realization of ordered mesostructures and potential applications in welded nanostructures, porous electrodes and ion sensors. In optical field, because the optical spectra of periodic sphere arrays are most sensitive to the lattice spacing of arrays, the assemblies show great potential applications in optical sensors, electrochromic and electromechanochromic inks, and so on [487-489]. Kim and co-workers [490] demonstrated the color of 'M-ink' can be tuned by varying the magnetic and photochemical parameters. Most recently, Dong's group [491] reported the self-assembly can be utilized to construct highly ordered mesoporous few-layer graphene framework from the $\mathrm{Fe}_{3} \mathrm{O}_{4}$ units. After selective etching the inner $\mathrm{Fe}_{3} \mathrm{O}_{4}$, the remaining ordered mesoporous graphene frameworks exhibited excellent cycling stability and rate performance for lithium-ion batteries.

\section{Seed-diffused method}

For seed growth process discussed in the above section, the adatoms almost nucleate on the surface of seeds for further growth. This situation can be understood by the smaller coordination number than that of atoms in the bulk, which will decrease much more surface energy. Unlikely, our group recently reported a seed-based diffusion route, achieving the diffusion of newly formed $\mathrm{Cu}$ atoms into $\mathrm{Au}$ seeds to form intermetallic $\mathrm{CuAu}$ and $\mathrm{Cu}_{3} \mathrm{Au}$ NCs [492],

$$
\mathrm{Au}+\mathrm{Cu}^{2+}+\mathrm{OA} / \mathrm{TOA} \stackrel{\Delta}{\rightarrow} \mathrm{Cu}_{3} \mathrm{Au} \text { or } \mathrm{CuAu} .
$$

According to the equation, the final products were prepared by heating a mixture of $\mathrm{Cu}\left(\mathrm{CH}_{3} \mathrm{COO}\right)_{2}$, OA, tri-noctylamine (TOA) and Au seeds at an specific temperature for nearly $2 \mathrm{~h}$. Due to the synergetic effect of TOA/OA and $\mathrm{Au}$ surface, the $\mathrm{Cu}^{2+}$ ions can be reduced to $\mathrm{Cu}$ atoms or small clusters on the surface of Au seeds. Driven by the heating energy at high temperature and the entropy exchange from the disorder alloy to order intermetallics, the reduced $\mathrm{Cu}$ atoms will collide with the active sites on $\mathrm{Au}$ surface and diffuse from the surface inward to the lattice of $\mathrm{Au}$ seeds, resulting in $\mathrm{CuAu}$ or $\mathrm{Cu}_{3} \mathrm{Au}$ intermetallics (Fig. 35). In a related work, Wang et al. [493] discovered the noble-metal-induced reduction (NMIR) process on Au sur- 

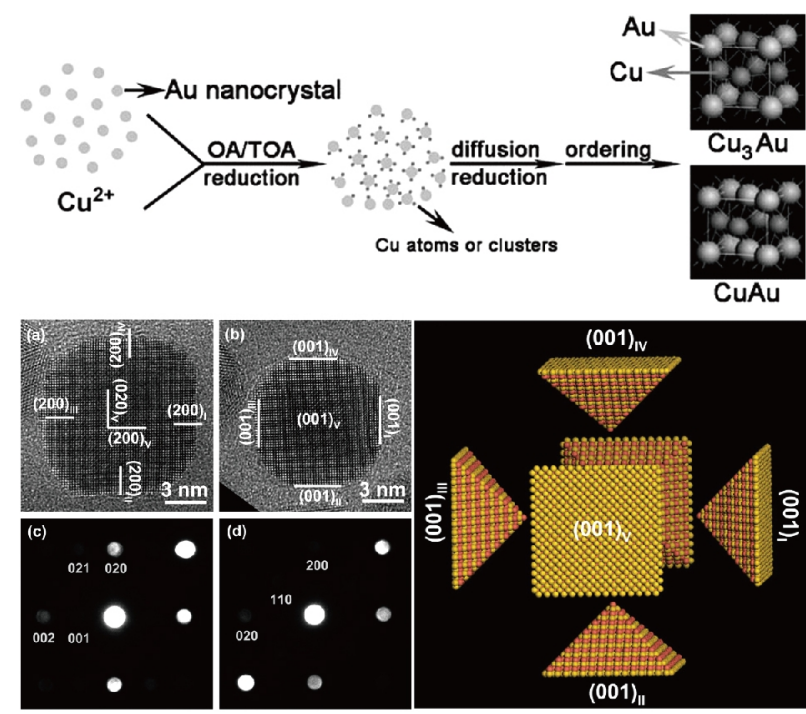

Figure 35 The top is the scheme of seed-based diffusion method for the formation of $\mathrm{CuAu}$ and $\mathrm{Cu}_{3} \mathrm{Au}$ intermetallics. Reproduced with permission from Ref. [492], Copyright 2010, WILEY-VCH Verlag GmbH \& Co. (a-d) HRTEM of multishell and corresponding electron nanodiffraction patterns of nano-onions for the sample of $\mathrm{CuAu}$ and $\mathrm{Cu}_{3} \mathrm{Au}$. Reproduced with permission from Ref. [494], Copyright 2010, American Physical Society.

face. Namely, in an alkyl amine system, the preformed $\mathrm{Au}$ seeds can facilitate the electron transfer from the amine to the late $3 \mathrm{~d}$ metals such as $\mathrm{Fe}, \mathrm{Co}$, and $\mathrm{Ni}$ ions and accelerate the reduction process. Although the reduction of transition-metal ions by amine is thermodynamically unfavorable, the coexistence of amine and noble metals can make the reduction happen. So, back to the former work, the reduction of $\mathrm{Cu}$ ions could also be facilitated by $\mathrm{Au}$ seeds and follow the NMIR process. Afterwards, Yu et al. [494] detailedly investigated the multishell intermetallic onions structure of the $\mathrm{Cu}$-Au intermetallics by atomically resolved HAADF-STEM and theoretical calculation (Figs $35 \mathrm{a}-\mathrm{d})$. Through DFT calculations, it is shown that the energy penalties for introducing antiphase boundaries, which are small for alloy systems compared to typical surface energies, results in the diffusion of $\mathrm{Cu}$ atoms into the NPs, making it feasible to prepare intermetallic onions. Actually, the diffusion of element with smaller atomic radius into the lattice with bigger ones is commonly in the nanoscaled metal carbides, borides, nitrides and phosphides [495]. In these materials, most of the metal-metalloid bonds contain some proportion of covalent component. In 2011, Wang et al. [496] firstly discovered the $\mathrm{Cu}_{3} \mathrm{~N}$ can be prepared by the decomposition of $\mathrm{Cu}\left(\mathrm{NO}_{3}\right)_{2}$ in the octadecylamine. They also investigated the effects of solvents, metal salts and so on for the formation of $\mathrm{Cu}_{3} \mathrm{~N}$. During the preparation of nitrides, the usage of $\mathrm{NH}_{3}$ is popular for the synthesis of transition metal nitrides nanostructures such as $\mathrm{VN}, \mathrm{Ni}_{3} \mathrm{~N}$, and $\mathrm{Ni}_{3} \mathrm{FeN}$ [496-500]. Similarly, some active $\mathrm{B}$-containing and $\mathrm{P}$-containing agents such as $\mathrm{NaBH}_{4}$, $\mathrm{NaH}_{2} \mathrm{PO}_{2}$, trioctylphosphine and so on have been developed to achieve the B-doped and P-doped transition metal borides and phosphides through the in-situ phase transfer process [501-503].

Recently, Niu et al. [504] confirmed this mechanism again and extended this method to the synthesis of highly branched Pt-Ni NCs enclosed by stepped surface of $\{112\}$ facets. Using the same shape of branch Pt as starting seeds, the Pt-Ni bimetallic nanobundles (NBs) with branched morphology and stepped surfaces have been prepared via the diffusion of $\mathrm{Ni}$ atoms. This mild diffusion process did not bring significant size and shape changes to the final bimetallic NCs versus the starting Pt NCs. The surface atomic steps can also remain after this structural evolution.

In summary, the seed-based diffusion method indeed offers a method to transfer the monometallic NCs to its corresponding bimetallic structures, while maintaining the comparable size and morphology. Further, the compositon of the as-prepared bimetallic structure can be tuned by the quantity of adatoms. The crystalline phase such as disordered alloy or ordered intermetallics can also be controlled by the heating temperature.

\section{TOP-DOWN AND BOTTOM-UP}

The top-down strategy is the dissolution of ordered structures such as crystalline NCs, assembly, polymers, superlattice into the smaller pieces such as ions, clusters, monomers, and so on. The emphasis of top-down strategy is the carving of nanostructure in the purpose of more complex structure with desired properties and more excellent behavior. So, the remaining residue is the focus in top-down strategy and the leaving counterpart is usually discarded. This section will briefly discuss the new emerging synthetic strategies involving the integration of top-down and following bottom-up process in the aim of utilizing the abandoned units. The re-organization of the leaving counterparts with the additional building blocks may bring new functionalities and properties in the as-obtained composites. To date, the study about this sequential strategy is still primary and the related mechanism is elusive.

For example, dealloying process of bimetallic NPs is an old and versatile top-down strategy to generate more reactive sites and carve the structure by selectively removing the useless part. Driven by the difference in the chemical 
reactivity of two metallic species, the dealloying process has gained great success in constructing hollow [505], porous [506], concave [383,507] and frame structures [238] with fascinating geometrical beauty and interesting chemical and physical properties. The generated reactive sites such as interior vacancies and surface defects are strictly related to the dissolution of more active metals and rearrangement of the reserving residue. However, there is an easily neglected drawback with dealloying process which cannot afford to ignore-no matter the dealloying process originates from the oxidative etching which usually utilizes oxidant and acid [508,509] or galvanic reaction which involves the replacement between two different metals [344,510], the active ones are consistently oxidized to cationic counterpart and abandoned. The dealloying usually undergoes a drastic process and the leaving ions are elusive to capture, resulting in the lack of effective strategy to utilize the abandoned ionic systems and maximize the atom utilization [511]. Most recently, Ren et al. [512] reported an incorporation of chemical etching of Pt-Ni alloy and in-situ precipitation of LDH to decorate nickel-aluminum layered double hydroxide (NiAl-LDH) onto the Pt-Ni alloy surface. By this method, not only the abandoned $\mathrm{Ni}^{2+}$ via dealloying process can be effectively utilized, but also the basic active sites can be integrated to metallic catalysts (Figs 36a-c). Three possible equations that match dependency and synchronization of chemical etching and precipitation from electron and species transfer were speculated as follows:

$$
\begin{gathered}
\frac{1}{2} \mathrm{O}_{2}+\mathrm{H}_{2} \mathrm{O}+2 \mathrm{e}^{-} \rightleftharpoons 2 \mathrm{OH}^{-}, \\
\mathrm{Ni}(0)-2 \mathrm{e}^{-} \rightleftharpoons \mathrm{Ni}^{2+}, \\
\mathrm{Ni}^{2+}+\mathrm{Al}^{3+}+2 \mathrm{OH}^{-}+3 \mathrm{NO}_{3}^{-} \rightleftharpoons\left[\mathrm{NiAl}(\mathrm{OH})_{2}\right]\left(\mathrm{NO}_{3}\right)_{3} .
\end{gathered}
$$

Equations (1) and (2) are two half reactions in oxidative etching process and related to the electron transfer from $\mathrm{Ni}(0)$ to oxygen. Equation (3) tells the nucleation process of NiAl-LDH. The NiAl-LDH growth is a thermodynamically spontaneous process which can break the equilibrium of etching reactions, thus drastically increasing the etching rate. It is deduced that the electron and species transfer in an orderly way between the two reactions, to a certain extent achieving synchronization. The related LDH-growth assisted etching of Pt-Ni NPs is schematically illustrated in Fig. 36c. Again, based on the same idea, this sequential top-down and bottom-up strategy can be generalized to

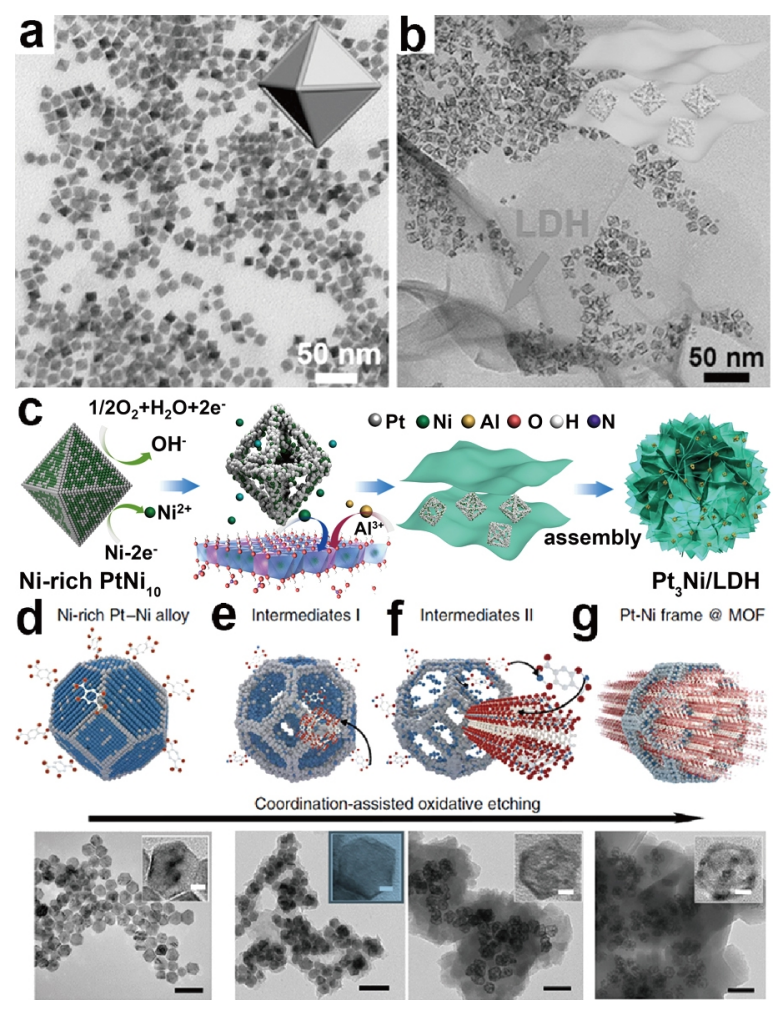

Figure 36 (a) TEM image and corresponding schematic model of initial solid $\mathrm{PtNi}_{10}$ octahedron; b) TEM image of hybrid $\mathrm{Pt}_{3} \mathrm{Ni} / \mathrm{LDH}$. (c) Schematic illustration of the proposed chemical etching mechanism of octahedral $\mathrm{PtNi}_{10}$ and formation of NiAl-LDH. Reproduced with permission from Ref. [512], Copyright 2015, WILEY-VCH Verlag GmbH \& Co. (d-g) Scheme and corresponding TEM images of the coordination-assisted oxidative etching process. Reproduced with permission from Ref. [129], Copyright 2015, Nature Publishing Group.

synthesize Pt-Ni frame@MOF frame and frame structures, which was reported by Li et al. [129] most recently. The PVP capped Ni-rich Pt-Ni truncated octahedron NCs were dispersed in DMF to form a turbid solution, followed by the addition of 2,5-dioxidoterephthalate solution. According to the mentioned mechanism, a well-defined MOF of $\mathrm{Ni}_{2}$ dobdc (dobdc 4 -(2,5-dioxidoterephthalate)), commonly known as Ni-MOF-74, was constructed on the surface of dealloyed Pt-Ni NCs. In detail, the top-down part including the oxidative etching of Pt-Ni alloy and the bottom-up part including the in-situ nucleation of MOF-74 are linked with each other. After the Pt-Ni polyhedra being etched to a Pt-rich bimetallic framework, another MOF containing the $\mathrm{Ni}$ anodes would immediately appear and cover the surface of bimetallic framework (Figs 36d-g).

\section{SUMMARY AND OUTLOOK}

Over a few decades, fruitful studies have been witnessed 
in the field of shape-controlled synthesis of NCs. However, these are mainly built upon the growth regime (at the scale $>1 \mathrm{~nm}$ ). To date, abundant theories have been established to explain the experimental results during the nucleation and growth process of NCs. Despite tremendous efforts have been paid, limited success have been achieved for the research systems whose main study subjects are at the scale $<1 \mathrm{~nm}$. This is mainly because the tracking, understanding, and controlling the nucleation are still elusive. Accompanying with the fast development of nanoscience, the properties of nanomaterials are usually found to be related to the sub-nano structures of single atoms, clusters, defects, segregation and so on. Studies focus on the realtime and real-space study of nucleation and growth processes are the keypoints for the nanoscience, cluster-science and single atom-science, which still remain great challenges. One obstacle is the shortage of experimental tools and well-established theory to capture, characterize, and monitor the nucleation which is decisive to the final product. Recently, some in-situ techniques such as in-situ EM and in-situ XAFS have gained outstanding improvement to endow the ability of following the nucleation process in liquid phase. We foresee the investigations of a broad set of scientific issues which fall between the homogeneous and heterogeneous fields especially in the sub-nano regime may bring new salient experimental and theoretical advances.

The advances of synthetic method, nucleation and growth mechanism and in-situ techniques will greatly improve our understanding of the controlled synthesis of nanomaterials which own the "smart" geometric and electronic structures. We believe the synthesis of nanostructure is case-by-case, which relies on the integration of several methods and basic principles. Especially the control of more complex and sophisticated structures, the successful preparation is strictly dependent on the advances of understanding of the nucleation and growth process.

\section{Received 12 August 2016; accepted 30 August 2016;} published online 14 November 2016

1 Wang X, Peng Q, Li Y. Interface-mediated growth of monodispersed nanostructures. Acc Chem Res, 2007, 40: 635-643

2 Xia Y, Xiong Y, Lim B, et al. Shape-controlled synthesis of metal nanocrystals: simple chemistry meets complex physics? Angew Chem Int Ed, 2009, 48: 60-103

3 Lim B, Xia Y. Metal nanocrystals with highly branched morphologies. Angew Chem Int Ed, 2011, 50: 76-85

4 Zhang H, Jin M, Xia Y. Noble-metal nanocrystals with concave surfaces: synthesis and applications. Angew Chem Int Ed, 2012, 51: 7656-7673

5 Xiong Y, Wiley BJ, Xia Y. Nanocrystals with unconventional shapes-a class of promising catalysts. Angew Chem Int Ed, 2007,
46: 7157-7159

6 Zhou ZY, Tian N, Li JT, et al. Nanomaterials of high surface energy with exceptional properties in catalysis and energy storage. Chem Soc Rev, 2011, 40: 4167-4185

7 Quan Z, Wang Y, Fang J. High-index faceted noble metal nanocrystals. Acc Chem Res, 2013, 46: 191-202

8 Chen M, Wu B, Yang J, et al. Small adsorbate-assisted shape control of Pd and Pt nanocrystals. Adv Mater, 2012, 24: 862-879

9 Liu Y, Goebl J, Yin Y. Templated synthesis of nanostructured materials. Chem Soc Rev, 2013, 42: 2610-2653

10 Chiu CY, Ruan L, Huang Y. Biomolecular specificity controlled nanomaterial synthesis. Chem Soc Rev, 2013, 42: 2512-2527

11 Shi W, Song S, Zhang H. Hydrothermal synthetic strategies of inorganic semiconducting nanostructures. Chem Soc Rev, 2013, 42: 5714-5743

12 Modeshia DR, Walton RI. Solvothermal synthesis of perovskites and pyrochlores: crystallisation of functional oxides under mild conditions. Chem Soc Rev, 2010, 39: 4303-4325

13 Nadagouda MN, Speth TF, Varma RS. Microwave-assisted green synthesis of silver nanostructures. Acc Chem Res, 2011, 44: $469-478$

$14 \mathrm{Xu} \mathrm{H}$, Zeiger BW, Suslick KS. Sonochemical synthesis of nanomaterials. Chem Soc Rev, 2013, 42: 2555-2567

15 Tong G, Guan J, Xiao Z, et al. In situ generated gas bubble-assisted modulation of the morphologies, photocatalytic, and magnetic properties of ferric oxide nanostructures synthesized by thermal decomposition of iron nitrate. J Nanopart Res, 2010, 12: 3025-3037

16 Stoner EC, Wohlfarth EP. A mechanism of magnetic hysteresis in heterogeneous alloys. Philos Trans R Soc A-Math Phys Eng Scis, 1948, 240: 599-642

$17 \mathrm{Ku}$ JY, Aruguete DM, Alivisatos AP, et al. Self-assembly of magnetic nanoparticles in evaporating solution. J Am Chem Soc, 2011, 133: $838-848$

18 Alphandéry E, Ding Y, Ngo AT, et al. Assemblies of aligned magnetotactic bacteria and extracted magnetosomes: what is the main factor responsible for the magnetic anisotropy? ACS Nano, 2009, 3: 1539-1547

19 Petit C, Russier V, Pileni MP. Effect of the structure of cobalt nanocrystal organization on the collective magnetic properties. J Phys Chem B, 2003, 107: 10333-10336

20 LaMer VK, Dinegar RH. Theory, production and mechanism of formation of monodispersed hydrosols. J Am Chem Soc, 1950, 72: 4847-4854

21 Auer S, Frenkel D. Prediction of absolute crystal-nucleation rate in hard-sphere colloids. Nature, 2001, 409: 1020-1023

22 Erdemir D, Lee AY, Myerson AS. Nucleation of crystals from solution: classical and two-step models. Acc Chem Res, 2009, 42: 621-629

23 Wolde PR. Enhancement of protein crystal nucleation by critical density fluctuations. Science, 1997, 277: 1975-1978

24 Nicolis G, Nicolis C. Enhancement of the nucleation of protein crystals by the presence of an intermediate phase: a kinetic model. Phys A-Statistical Mech its Appl, 2003, 323: 139-154

25 Gavezzotti A. Molecular aggregation of acetic acid in a carbon tetrachloride solution: a molecular dynamics study with a view to crystal nucleation. Chem Eur J, 1999, 5: 567-576

26 Talanquer V, Oxtoby DW. Crystal nucleation in the presence of a metastable critical point. J Chem Phys, 1998, 109: 223-227

27 Peng X, Wickham J, Alivisatos AP. Kinetics of II-VI and III-V colloidal semiconductor nanocrystal growth: "focusing" of size distributions. J Am Chem Soc, 1998, 120: 5343-5344 
28 Peng ZA, Peng X. Mechanisms of the shape evolution of CdSe nanocrystals. J Am Chem Soc, 2001, 123: 1389-1395

29 Peng ZA, Peng X. Nearly monodisperse and shape-controlled CdSe nanocrystals via alternative routes: nucleation and growth. J Am Chem Soc, 2002, 124: 3343-3353

30 Thessing J, Qian J, Chen H, et al. Interparticle influence on size/size distribution evolution of nanocrystals. J Am Chem Soc, 2007, 129: 2736-2737

31 Lin Z, Gilbert B, Liu Q, et al. A thermodynamically stable nanophase material. J Am Chem Soc, 2006, 128: 6126-6131

32 Zhang J, Lin Z, Lan Y, et al. A multistep oriented attachment kinetics: coarsening of $\mathrm{ZnS}$ nanoparticle in concentrated $\mathrm{NaOH}$. J Am Chem Soc, 2006, 128: 12981-12987

33 Li S, Xie T, Peng Q, et al. Nucleation and growth of $\mathrm{CeF}_{3}$ and $\mathrm{NaCeF}_{4}$ nanocrystals. Chem Eur J, 2009, 15: 2512-2517

34 Xie $\mathrm{T}, \mathrm{Li}$, Peng Q, et al. Monodisperse $\mathrm{BaF}_{2}$ nanocrystals: phases, size transitions, and self-assembly. Angew Chem Int Ed, 2009, 48: 196-200

35 Xie T, Li S, Wang W, et al. Nucleation and growth of $\mathrm{BaF} \mathrm{Cl}_{2-x}$ nanorods. Chem Eur J, 2008, 14: 9730-9735

36 Wu Y, Wang D, Li Y. Nanocrystals from solutions: catalysts. Chem Soc Rev, 2014, 43: 2112-2124

37 Hulliger J. Chemistry and crystal growth. Angew Chem Int Ed, 1994, 33: 143-162

38 Klionsky DJ, Abdalla FC, Abeliovich H, et al. Guidelines for the use and interpretation of assays for monitoring autophagy. Autophagy, 2002, 8: 445-544

39 Manna L, Scher EC, Alivisatos AP. Synthesis of soluble and processable rod-, arrow-, teardrop-, and tetrapod-shaped CdSe nanocrystals. J Am Chem Soc, 2000, 122: 12700-12706

40 Peng ZA, Peng X. Formation of high-quality CdTe, CdSe, and CdS nanocrystals using $\mathrm{CdO}$ as precursor. J Am Chem Soc, 2001, 123: 183-184

41 Lee JH, Huh YM, Jun Y, et al. Artificially engineered magnetic nanoparticles for ultra-sensitive molecular imaging. Nat Med, 2007, 13: 95-99

42 Dai Y, Zhang Y, Li QK, et al. Synthesis and optical properties of tetrapod-like zinc oxide nanorods. Chem Phys Lett, 2002, 358: 83-86

43 Tao A, Sinsermsuksakul P, Yang P. Polyhedral silver nanocrystals with distinct scattering signatures. Angew Chem Int Ed, 2006, 45: 4597-4601

44 Xia X, Zeng J, McDearmon B, et al. Silver nanocrystals with concave surfaces and their optical and surface-enhanced raman scattering properties. Angew Chem, 2011, 123: 12750-12754

45 Tian N, Zhou ZY, Sun SG, et al. Synthesis of tetrahexahedral platinum nanocrystals with high-index facets and high electro-oxidation activity. Science, 2007, 316: 732-735

46 Fan Z, Huang X, Han Y, et al. Surface modification-induced phase transformation of hexagonal close-packed gold square sheets. Nat Commun, 2015, 6: 6571

47 Bort $\mathrm{H}$, Jüttner K, Lorenz WJ, et al. Underpotential alloy formation in the system $\mathrm{Ag}(h k l) / \mathrm{Cd}^{2+}$. Electrochim Acta, 1983, 28: 993-1001

48 Wang Y, Wan D, Xie S, et al. Synthesis of silver octahedra with controlled sizes and optical properties via seed-mediated growth. ACS Nano, 2013, 7: 4586-4594

49 Huang X, Qi X, Boey F, et al. Graphene-based composites. Chem Soc Rev, 2012, 41: 666-686

50 Huang X, Zeng Z, Zhang H. Metal dichalcogenide nanosheets: preparation, properties and applications. Chem Soc Rev, 2013, 42: 1934-1946

51 Allen MJ, Tung VC, Kaner RB. Honeycomb carbon: a review of graphene. Chem Rev, 2010, 110: 132-145

52 Haruta M. Gold catalysts prepared by coprecipitation for low-temperature oxidation of hydrogen and of carbon monoxide. J Catal, 1989, 115: 301-309

53 Valden M. Onset of catalytic activity of gold clusters on titania with the appearance of nonmetallic properties. Science, 1998, 281: $1647-1650$

54 Chen D, Feng H, Li J. Graphene oxide: preparation, functionalization, and electrochemical applications. Chem Rev, 2012, 112: 6027-6053

55 Zhao G, Schwartz Z, Wieland M, et al. High surface energy enhances cell response to titanium substrate microstructure. J Biomed Mater Res A, 2005, 74A: 49-58

56 Dreyer DR, Park S, Bielawski CW, et al. The chemistry of graphene oxide. Chem Soc Rev, 2010, 39: 228-240

57 Loh KP, Bao Q, Eda G, et al. Graphene oxide as a chemically tunable platform for optical applications. Nat Chem, 2010, 2: 1015-1024

58 Zhang $\mathrm{H}$, Jin $\mathrm{M}$, Xiong $\mathrm{Y}$, et al. Shape-controlled synthesis of Pd nanocrystals and their catalytic applications. Acc Chem Res, 2013, 46: $1783-1794$

59 Schwartz-Duval AS, Misra SK, Mukherjee P, et al. An anisotropic propagation technique for synthesizing hyperbranched polyvillic gold nanoparticles. Nano Res, 2016

60 Fang Z, Zhang Y, Du F, et al. Growth of anisotropic platinum nanostructures catalyzed by gold seed nanoparticles. Nano Res, 2008, 1: 249-257

$61 \mathrm{Xu} \mathrm{X}, \mathrm{Lu} \mathrm{Y}$, Yang $\mathrm{Y}$, et al. Tuning the growth of metal-organic framework nanocrystals by using polyoxometalates as coordination modulators. Sci China Mater, 2015, 58: 370-377

62 Niu Z, Li Y. Removal and utilization of capping agents in nanocatalysis. Chem Mater, 2014, 26: 72-83

63 Huang X, Tang S, Mu X, et al. Freestanding palladium nanosheets with plasmonic and catalytic properties. Nat Nanotech, 2011, 6: 28-32

64 Zeng J, Zheng Y, Rycenga M, et al. Controlling the shapes of silver nanocrystals with different capping agents. J Am Chem Soc, 2010, 132: $8552-8553$

65 Dou L, Wong AB, Yu Y, et al. Atomically thin two-dimensional organic-inorganic hybrid perovskites. Science, 2015, 349: 1518-1521

66 Wang ZL. Transmission electron microscopy of shape-controlled nanocrystals and their assemblies. J Phys Chem B, 2000, 104: 1153-1175

67 Wiley B, Sun Y, Mayers B, et al. Shape-controlled synthesis of metal nanostructures: the case of silver. Chem Eur J, 2005, 11: 454-463

68 Nikoobakht B, El-Sayed MA. Preparation and growth mechanism of gold nanorods (nrs) using seed-mediated growth method. Chem Mater, 2003, 15: 1957-1962

69 Wadams RC, Fabris L, Vaia RA, et al. Time-dependent susceptibility of the growth of gold nanorods to the addition of a cosurfactant. Chem Mater, 2013, 25: 4772-4780

70 Ye X, Jin L, Caglayan $\mathrm{H}$, et al. Improved size-tunable synthesis of monodisperse gold nanorods through the use of aromatic additives. ACS Nano, 2012, 6: 2804-2817

71 Eustis S, El-Sayed MA. Why gold nanoparticles are more precious than pretty gold: noble metal surface plasmon resonance and its enhancement of the radiative and nonradiative properties of nanocrystals of different shapes. Chem Soc Rev, 2006, 35: 209-217

72 Murphy CJ, Gole AM, Stone JW, et al. Gold nanoparticles in biology: beyond toxicity to cellular imaging. Acc Chem Res, 2008, 41: $1721-1730$

73 Dickerson EB, Dreaden EC, Huang X, et al. Gold nanorod assisted near-infrared plasmonic photothermal therapy (PPTT) of squa- 
mous cell carcinoma in mice. Cancer Lett, 2008, 269: 57-66

$74 \mathrm{Hu}$ M, Chen J, Li ZY, et al. Gold nanostructures: engineering their plasmonic properties for biomedical applications. Chem Soc Rev, 2006, 35: 1084-1094

75 Ghosh SK, Pal T. Interparticle coupling effect on the surface plasmon resonance of gold nanoparticles: from theory to applications. Chem Rev, 2007, 107: 4797-4862

76 Jones MR, Osberg KD, Macfarlane RJ, et al. Templated techniques for the synthesis and assembly of plasmonic nanostructures. Chem Rev, 2011, 111: 3736-3827

77 Xiong Y, Cai H, Wiley BJ, et al. Synthesis and mechanistic study of palladium nanobars and nanorods. J Am Chem Soc, 2007, 129: 3665-3675

78 Huang X, Zhang H, Guo C, et al. Simplifying the creation of hollow metallic nanostructures: one-pot synthesis of hollow palladium/platinum single-crystalline nanocubes. Angew Chem, 2009, 121: 4902-4906

79 Li C, Sato R, Kanehara M, et al. Controllable polyol synthesis of uniform palladium icosahedra: effect of twinned structure on deformation of crystalline lattices. Angew Chem, 2009, 121: 7015-7019

80 Huang X, Zheng N. One-pot, high-yield synthesis of 5-fold twinned Pd nanowires and nanorods. J Am Chem Soc, 2009, 131: 4602-4603

81 Bratlie KM, Lee H, Komvopoulos K, et al. Platinum nanoparticle shape effects on benzene hydrogenation selectivity. Nano Lett, 2007, 7: 3097-3101

82 Sneed BT, Kuo CH, Brodsky CN, et al. Iodide-mediated control of rhodium epitaxial growth on well-defined noble metal nanocrystals: synthesis, characterization, and structure-dependent catalytic properties. J Am Chem Soc, 2012, 134: 18417-18426

83 Sneed BT, Brodsky CN, Kuo CH, et al. Nanoscale-phase-separated $\mathrm{Pd}-\mathrm{Rh}$ boxes synthesized via metal migration: an archetype for studying lattice strain and composition effects in electrocatalysis. J Am Chem Soc, 2013, 135: 14691-14700

84 Yin AX, Liu WC, Ke J, et al. Ru nanocrystals with shape-dependent surface-enhanced Raman spectra and catalytic properties: controlled synthesis and DFT calculations. J Am Chem Soc, 2012, 134: 20479-20489

85 Gu J, Zhang YW, Tao FF. Shape control of bimetallic nanocatalysts through well-designed colloidal chemistry approaches. Chem Soc Rev, 2012, 41: 8050-8065

$86 \mathrm{Yu} \mathrm{JH}$, Joo J, Park HM, et al. Synthesis of quantum-sized cubic ZnS nanorods by the oriented attachment mechanism. J Am Chem Soc, 2005, 127: 5662-5670

87 Huang X, Li S, Huang Y, et al. Synthesis of hexagonal close-packed gold nanostructures. Nat Commun, 2011, 2: 292

88 Zitoun D, Pinna N, Frolet N, et al. Single crystal manganese oxide multipods by oriented attachment. J Am Chem Soc, 2005, 127: 15034-15035

89 Penn RL. Imperfect oriented attachment: dislocation generation in defect-free nanocrystals. Science, 1998, 281: 969-971

90 Penn RL, Banfield JF. Oriented attachment and growth, twinning, polytypism, and formation of metastable phases; insights from nanocrystalline $\mathrm{TiO}_{2}$. Am Miner, 1998, 83: 1077-1082

91 Pacholski C, Kornowski A, Weller H. Self-assembly of ZnO: from nanodots to nanorods. Angew Chem Int Ed, 2002, 41: 1188-1191

92 Banfield JF. Aggregation-based crystal growth and microstructure development in natural iron oxyhydroxide biomineralization products. Science, 2000, 289: 751-754

93 Cho KS, Talapin DV, Gaschler W, et al. Designing PbSe nanowires and nanorings through oriented attachment of nanoparticles. J Am
Chem Soc, 2005, 127: 7140-7147

94 Halder A, Ravishankar N. Ultrafine single-crystalline gold nanowire arrays by oriented attachment. Adv Mater, 2007, 19: 1854-1858

95 Zhang Q, Liu SJ, Yu SH. Recent advances in oriented attachment growth and synthesis of functional materials: concept, evidence, mechanism, and future. J Mater Chem, 2009, 19: 191-207

96 Zhang X, Xie Y. Recent advances in free-standing two-dimensional crystals with atomic thickness: design, assembly and transfer strategies. Chem Soc Rev, 2013, 42: 8187-8199

97 Zhang X, Zhang J, Zhao J, et al. Half-metallic ferromagnetism in synthetic $\mathrm{Co}_{9} \mathrm{Se}_{8}$ nanosheets with atomic thickness. J Am Chem Soc, 2012, 134: 11908-11911

98 Schliehe $\mathrm{C}$, Juarez $\mathrm{BH}$, Pelletier $\mathrm{M}$, et al. Ultrathin $\mathrm{PbS}$ sheets by two-dimensional oriented attachment. Science, 2010, 329: 550-553

99 Duan H, Yan N, Yu R, et al. Ultrathin rhodium nanosheets. Nat Commun, 2014, 5: 3093

100 Hu S, Wang X. Ultrathin nanostructures: smaller size with new phenomena. Chem Soc Rev, 2013, 42: 5577-5594

101 Delahay P, Tobias CW, Gerischer H. Advances in Electrochemistry and Electrochemical Engineering, Vol. 11. New York: John Wiley and Sons, 1978: 446

102 Sudha V, Sangaranarayanan MV. Underpotential deposition of metals: structural and thermodynamic considerations. J Phys Chem B, 2002, 106: 2699-2707

103 Bockris JOM, Reddy AKN. Modern Electrochemistry: an Introduction to an Interdisciplinary Area. Berlin: Springer Science \& Business Media, 2012

104 Personick ML, Langille MR, Zhang J, et al. Shape control of gold nanoparticles by silver underpotential deposition. Nano Lett, 2011, 11: 3394-3398

105 Zhang L, Zhang J, Kuang Q, et al. $\mathrm{Cu}^{2+}$-assisted synthesis of hexoctahedral Au-Pd alloy nanocrystals with high-index facets. J Am Chem Soc, 2011, 133: 17114-17117

106 Herrero E, Buller LJ, Abruña HD. Underpotential deposition at single crystal surfaces of $\mathrm{Au}, \mathrm{Pt}, \mathrm{Ag}$ and other materials. Chem Rev, 2001, 101: 1897-1930

107 Jackson SR, McBride JR, Rosenthal SJ, et al. Where's the silver? Imaging trace silver coverage on the surface of gold nanorods. J Am Chem Soc, 2014, 136: 5261-5263

108 Ge J, He D, Bai L, et al. Ordered porous Pd octahedra covered with monolayer Ru atoms. J Am Chem Soc, 2015, 137: 14566-14569

109 Sun S. Monodisperse FePt nanoparticles and ferromagnetic FePt nanocrystal superlattices. Science, 2000, 287: 1989-1992

110 Fan Z, Bosman M, Huang X, et al. Stabilization of $4 \mathrm{H}$ hexagonal phase in gold nanoribbons. Nat Commun, 2015, 6: 7684

111 Liu X, Luo J, Zhu J. Size effect on the crystal structure of silver nanowires. Nano Lett, 2006, 6: 408-412

112 Ling T, Xie L, Zhu J, et al. Icosahedral face-centered cubic Fe nanoparticles: facile synthesis and characterization with aberration-corrected TEM. Nano Lett, 2009, 9: 1572-1576

113 Kim C, Kim C, Lee K, et al. Shaped Ni nanoparticles with an unconventional hcp crystalline structure. Chem Commun, 2014, 50 6353-6356

114 Kusada K, Kobayashi H, Yamamoto T, et al. Discovery of face-centered-cubic ruthenium nanoparticles: facile size-controlled synthesis using the chemical reduction method. J Am Chem Soc, 2013, 135: 5493-5496

115 Li Y, Cheng H, Yao T, et al. Hexane-driven icosahedral to cuboctahedral structure transformation of gold nanoclusters. J Am Chem Soc, 2012, 134: 17997-18003 
116 Fan Z, Zhu Y, Huang X, et al. Synthesis of ultrathin face-centered-cubic Au@Pt and Au@Pd core-shell nanoplates from hexagonal-close-packed Au square sheets. Angew Chem Int Ed, 2015, 54: $5672-5676$

117 Zhang S, Guo S, Zhu H, et al. Structure-induced enhancement in electrooxidation of trimetallic FePtAu nanoparticles. J Am Chem Soc, 2012, 134: 5060-5063

118 Yao Y, He DS, Lin Y, et al. Modulating fcc and hcp ruthenium on the surface of palladium-copper alloy through tunable lattice mismatch. Angew Chem Int Ed, 2016, 55: 5501-5505

119 Fan Z, Zhang H. Crystal phase-controlled synthesis, properties and applications of noble metal nanomaterials. Chem Soc Rev, 2016, 45: $63-82$

120 Langille MR, Personick ML, Zhang J, et al. Bottom-up synthesis of gold octahedra with tailorable hollow features. J Am Chem Soc, 2011, 133: 10414-10417

121 Wang Y, Xia Y. Bottom-up and top-down approaches to the synthesis of monodispersed spherical colloids of low melting-point metals. Nano Lett, 2004, 4: 2047-2050

122 Zhang G, Jin X, Li H, et al. N-doped crumpled graphene: bottom-up synthesis and its superior oxygen reduction performance. Sci China Mater, 2016, 59: 337-347

123 Zhuang Z, Peng Q, Li Y. Controlled synthesis of semiconductor nanostructures in the liquid phase. Chem Soc Rev, 2011, 40: 5492-5513

124 Lesnyak V, Gaponik N, Eychmüller A. Colloidal semiconductor nanocrystals: the aqueous approach. Chem Soc Rev, 2013, 42: 2905-2929

125 Kershaw SV, Susha AS, Rogach AL. Narrow bandgap colloidal metal chalcogenide quantum dots: synthetic methods, heterostructures, assemblies, electronic and infrared optical properties. Chem Soc Rev, 2013, 42: 3033-3087

126 Gao MR, Xu YF, Jiang J, et al. Nanostructured metal chalcogenides: synthesis, modification, and applications in energy conversion and storage devices. Chem Soc Rev, 2013, 42: 2986-3017

127 Nirmal M, Dabbousi BO, Bawendi MG, et al. Fluorescence intermittency in single cadmium selenide nanocrystals. Nature, 1996, 383: 802-804

128 Ekimov AI, Efros AL, Onushchenko AA. Quantum size effect in semiconductor microcrystals. Solid State Commun, 1985, 56: 921-924

129 Li Z, Yu R, Huang J, et al. Platinum-nickel frame within metalorganic framework fabricated in situ for hydrogen enrichment and molecular sieving. Nat Commun, 2015, 6: 8248

130 Zhuang Z, Peng Q, Wang X, et al. Tetrahedral colloidal crystals of $\mathrm{Ag}_{2} \mathrm{~S}$ nanocrystals. Angew Chem, 2007, 119: 8322-8325

131 Zhuang Z, Peng Q, Zhang B, et al. Controllable synthesis of $\mathrm{Cu}_{2} \mathrm{~S}$ nanocrystals and their assembly into a superlattice. J Am Chem Soc, 2008, 130: 10482-10483

132 Liu Y, Deng Y, Sun Z, et al. Hierarchical $\mathrm{Cu}_{2} \mathrm{~S}$ microsponges constructed from nanosheets for efficient photocatalysis. Small, 2013, 9: $2702-2708$

133 Jiao S, Xu L, Jiang K, et al. Well-defined non-spherical copper sulfide mesocages with single-crystalline shells by shape-controlled $\mathrm{Cu}_{2} \mathrm{O}$ crystal templating. Adv Mater, 2006, 18: 1174-1177

134 Akkerman QA, Genovese A, George C, et al. From binary $\mathrm{Cu}_{2} \mathrm{~S}$ to ternary $\mathrm{Cu}-\mathrm{In}-\mathrm{S}$ and quaternary $\mathrm{Cu}-\mathrm{In}-\mathrm{Zn}-\mathrm{S}$ nanocrystals with tunable composition via partial cation exchange. ACS Nano, 2015, 9: $521-531$

135 Mews A, Eychmueller A, Giersig M, et al. Preparation, characterization, and photophysics of the quantum dot quantum well system cadmium sulfide/mercury sulfide/cadmium sulfide. J Phys Chem,
1994, 98: 934-941

136 Wang X, Liu M, Zhou Z, et al. Toward facet engineering of CdS nanocrystals and their shape-dependent photocatalytic activities. J Phys Chem C, 2015, 119: 20555-20560

137 Ma L, Liang S, Liu XL, et al. Synthesis of dumbbell-like gold-metal sulfide core-shell nanorods with largely enhanced transverse plasmon resonance in visible region and efficiently improved photocatalytic activity. Adv Funct Mater, 2015, 25: 898-904

138 Dutta S, Ray C, Mallick S, et al. A gel-based approach to design hierarchical $\mathrm{CuS}$ decorated reduced graphene oxide nanosheets for enhanced peroxidase-like activity leading to colorimetric detection of dopamine. J Phys Chem C, 2015, 119: 23790-23800

139 Zhao B, Shao G, Fan B, et al. Synthesis of flower-like CuS hollow microspheres based on nanoflakes self-assembly and their microwave absorption properties. J Mater Chem A, 2015, 3: 10345-10352

140 Deng C, Ge X, Hu H, et al. Template-free and green sonochemical synthesis of hierarchically structured CuS hollow microspheres displaying excellent Fenton-like catalytic activities. CrystEngComm, 2014, 16: 2738-2745

141 Huang WC, Lyu LM, Yang YC, et al. Synthesis of $\mathrm{Cu}_{2} \mathrm{O}$ nanocrystals from cubic to rhombic dodecahedral structures and their comparative photocatalytic activity. J Am Chem Soc, 2012, 134: 1261-1267

142 Shang Y, Shao YM, Zhang DF, et al. Recrystallization-induced selfassembly for the growth of $\mathrm{Cu}_{2} \mathrm{O}$ superstructures. Angew Chem Int Ed, 2014, 53: 11514-11518

143 Hung LI, Tsung CK, Huang W, et al. Room-temperature formation of hollow $\mathrm{Cu}_{2} \mathrm{O}$ nanoparticles. Adv Mater, 2010, 22: 1910-1914

144 Wu J, Ren Z, Du S, et al. A highly active oxygen evolution electrocatalyst: ultrathin $\mathrm{CoNi}$ double hydroxide/CoO nanosheets synthesized via interface-directed assembly. Nano Res, 2016, 9 : 713-725

145 Wang Q, O'Hare D. Recent advances in the synthesis and application of layered double hydroxide (LDH) nanosheets. Chem Rev, 2012, 112: 4124-4155

146 Xu ZP, Zhang J, Adebajo MO, et al. Catalytic applications of layered double hydroxides and derivatives. Appl Clay Sci, 2011, 53: 139-150

147 Gong M, Dai H. A mini review of NiFe-based materials as highly active oxygen evolution reaction electrocatalysts. Nano Res, 2015, 8: 23-39

148 Wang H, Yan Y, Li B, et al. Hierarchical self-assembly of surfactantencapsulated and organically grafted polyoxometalate complexes. Chem Eur J, 2011, 17: 4273-4282

149 Li JR, Sculley J, Zhou HC. Metal-organic frameworks for separations. Chem Rev, 2012, 112: 869-932

$150 \mathrm{Lu} \mathrm{G}, \mathrm{Li}$ S, Guo Z, et al. Imparting functionality to a metal-organic framework material by controlled nanoparticle encapsulation. Nat Chem, 2012, 4: 310-316

151 Yang J, Zhang F, Lu H, et al. Hollow Zn/Co ZIF particles derived from core-shell ZIF-67@ZIF-8 as selective catalyst for the semi-hydrogenation of acetylene. Angew Chem Int Ed, 2015, 54: 10889-10893

152 Song YF, Tsunashima R. Recent advances on polyoxometalate-based molecular and composite materials. Chem Soc Rev, 2012, 41: 7384-7402

153 Nyman M, Burns PC. A comprehensive comparison of transition-metal and actinyl polyoxometalates. Chem Soc Rev, 2012, 41 7354-7367

154 Proust A, Matt B, Villanneau R, et al. Functionalization and postfunctionalization: a step towards polyoxometalate-based materials. Chem Soc Rev, 2012, 41: 7605-7622 
155 Banerjee A, Bassil BS, Röschenthaler GV, et al. Diphosphates and diphosphonates in polyoxometalate chemistry. Chem Soc Rev, 2012, 41: 7590-7604

156 Wang Y, Weinstock IA. Polyoxometalate-decorated nanoparticles. Chem Soc Rev, 2012, 41: 7479-7496

157 Miras HN, Yan J, Long DL, et al. Engineering polyoxometalates with emergent properties. Chem Soc Rev, 2012, 41: 7403-7430

158 Yin P, Li D, Liu T. Solution behaviors and self-assembly of polyoxometalates as models of macroions and amphiphilic polyoxometalate-organic hybrids as novel surfactants. Chem Soc Rev, 2012, 41: 7368-7383

$159 \mathrm{Li} \mathrm{H}$, Yang Y, Wang Y, et al. In situ fabrication of flower-like gold nanoparticles in surfactant-polyoxometalate-hybrid spherical assemblies. Chem Commun, 2010, 46: 3750-3752

160 Pradeep CP, Li FY, Lydon C, et al. Design and synthesis of "dumbbell" and "triangular" inorganic-organic hybrid nanopolyoxometalate clusters and their characterisation through ESI-MS analyses. Chem Eur J, 2011, 17: 7472-7479

161 Li Q, Wang E, Li S, et al. Template-free polyoxometalate-assisted synthesis for ZnO hollow spheres. J Solid State Chem, 2009, 182: 1149-1155

162 Azumi MA, Ishihara T, Nishiguchi $\mathrm{H}$, et al. Electrochemical intercalation of Li into heteropoly 12 molybdophosphoric acid ion-exchanged with Cs. Electrochemistry, 2002, 11: 869-874

$163 \mathrm{He}$, Xu B, Wang P, et al. A monolayer polyoxometalate superlattice. Adv Mater, 2014, 26: 4339-4344

164 Lee JY, Farha OK, Roberts J, et al. Metal-organic framework materials as catalysts. Chem Soc Rev, 2009, 38: 1450-1459

165 Schüth F, Sing KSW, Weitkamp J. Handbook of Porous Solids. Berlin: Wiley-VCH, 2002

166 Davis ME. Ordered porous materials for emerging applications. Nature, 2002, 417: 813-821

167 Zhou HC, Long JR, Yaghi OM. Introduction to metal-organic frameworks. Chem Rev, 2012, 112: 673-674

168 Xu R, Pang W, Yu J, et al. Chemistry of Zeolites and Related Porous Materials: Synthesis and Structure. Berlin: John Wiley \& Sons, 2009

169 Bansal RC, Goyal M. Activated Carbon Adsorption. Boca Raton: CRC press, 2005

170 Perry IV JJ, Perman JA, Zaworotko MJ. Design and synthesis of metal-organic frameworks using metal-organic polyhedra as supermolecular building blocks. Chem Soc Rev, 2009, 38: 1400-1417

171 Yaghi OM, O'Keeffe M, Ockwig NW, et al. Reticular synthesis and the design of new materials. Nature, 2003, 423: 705-714

$172 \mathrm{Li} \mathrm{CP}, \mathrm{Du} \mathrm{M}$. Role of solvents in coordination supramolecular systems. Chem Commun, 2011, 47: 5958-5972

173 Guillerm V, Kim D, Eubank JF, et al. A supermolecular building approach for the design and construction of metal-organic frameworks. Chem Soc Rev, 2014, 43: 6141-6172

174 Moon HR, Lim DW, Suh MP. Fabrication of metal nanoparticles in metal-organic frameworks. Chem Soc Rev, 2013, 42: 1807-1824

175 Zhu QL, Xu Q. Metal-organic framework composites. Chem Soc Rev, 2014, 43: 5468-5512

176 Liu XJ, Cui CH, Yu SH, et al. Pt-Ni alloyed nanocrystals with controlled architectures for enhanced methanol oxidation. Chem Comm, 2013, 49: 8703-8706

177 Chen Z, Waje M, Li W, et al. Supportless Pt and PtPd nanotubes as electrocatalysts for oxygen-reduction reactions. Angew Chem Int Ed, 2007, 46: 4060-4063

178 Ponrouch A, Garbarino S, Guay D. Effect of the nanostructure on the $\mathrm{CO}$ poisoning rate of platinum. Electrochemistry Commun, 2009, 11: 834-837
179 Colombi Ciacchi L, Pompe W, De Vita A. Growth of platinum clusters via addition of $\mathrm{Pt}(\mathrm{II})$ complexes: a first principles investigation. J Phys Chem B, 2003, 107: 1755-1764

180 Xia BY, Wu HB, Li N, et al. One-pot synthesis of Pt-Co alloy nanowire assemblies with tunable composition and enhanced electrocatalytic properties. Angew Chem, 2015, 127: 3868-3872

181 Zeng J, Lee J. Effects of preparation conditions on performance of carbon-supported nanosize Pt-Co catalysts for methanol electro-oxidation under acidic conditions. J Power Sources, 2005, 140: 268-273

182 Teng X, Liang X, Maksimuk S, et al. Synthesis of porous platinum nanoparticles. Small, 2006, 2: 249-253

183 Watt J, Cheong S, Toney MF, et al. Ultrafast growth of highly branched palladium nanostructures for catalysis. ACS Nano, 2010, 4: 396-402

184 Chen YH, Hung HH, Huang MH. Seed-mediated synthesis of palladium nanorods and branched nanocrystals and their use as recyclable Suzuki coupling reaction catalysts. J Am Chem Soc, 2009, 131: 9114-9121

185 Hao E, Bailey RC, Schatz GC, et al. Synthesis and optical properties of "branched" gold nanocrystals. Nano Lett, 2004, 4: 327-330

186 Lim B, Jiang M, Tao J, et al. Shape-controlled synthesis of Pd nanocrystals in aqueous solutions. Adv Funct Mater, 2009, 19: $189-200$

187 Nam KM, Shim JH, Ki H, et al. Single-crystalline hollow facecentered-cubic cobalt nanoparticles from solid face-centered-cubic cobalt oxide nanoparticles. Angew Chem Int Ed, 2008, 47: 9504-9508

188 Wang Y, Camargo PHC, Skrabalak SE, et al. A facile, water-based synthesis of highly branched nanostructures of silver. Langmuir, 2008, 24: 12042-12046

189 Wang X, Itoh $\mathrm{H}$, Naka K, et al. Tetrathiafulvalene-assisted formation of silver dendritic nanostructures in acetonitrile. Langmuir, 2003, 19: 6242-6246

190 Wen X, Xie YT, Mak WC, et al. Dendritic nanostructures of silver: facile synthesis, structural characterizations, and sensing applications. Langmuir, 2006, 22: 4836-4842

191 Hoefelmeyer JD, Niesz K, Somorjai GA, et al. Radial anisotropic growth of rhodium nanoparticles. Nano Lett, 2005, 5: 435-438

192 Xiong Y, Chen J, Wiley B, et al. Size-dependence of surface plasmon resonance and oxidation for Pd nanocubes synthesized via a seed etching process. Nano Lett, 2005, 5: 1237-1242

193 Lim B, Xiong Y, Xia Y. A water-based synthesis of octahedral, decahedral, and icosahedral Pd nanocrystals. Angew Chem, 2007, 119: 9439-9442

194 Jin M, Zhang H, Xie Z, et al. Palladium nanocrystals enclosed by $\{100\}$ and $\{111\}$ facets in controlled proportions and their catalytic activities for formic acid oxidation. Energy Environ Sci, 2012, 5: 6352-6357

$195 \mathrm{Lu}$ N, Chen W, Fang G, et al. 5-Fold twinned nanowires and single twinned right bipyramids of Pd: utilizing small organic molecules to tune the etching degree of $\mathrm{O}_{2} /$ halides. Chem Mater, 2014, 26: 2453-2459

196 Kimura F, Khalil G, Zettsu N, et al. Dual luminophore polystyrene microspheres for pressure-sensitive luminescent imaging. Meas Sci Technol, 2006, 17: 1254-1260

197 Humphrey SM, Grass ME, Habas SE, et al. Rhodium nanoparticles from cluster seeds: control of size and shape by precursor addition rate. Nano Lett, 2007, 7: 785-790

198 Peng Z, Yang H. PtAu bimetallic heteronanostructures made by post-synthesis modification of Pt-on-Au nanoparticles. Nano Res, 2009, 2: 406-415 
uvihill MJ, Ling XY, Henzie J, et al. Anisotropic etching of silver nanoparticles for plasmonic structures capable of single-particle SERS. J Am Chem Soc, 2010, 132: 268-274

200 Peng Z, Yang H. Synthesis and oxygen reduction electrocatalytic property of Pt-on-Pd bimetallic heteronanostructures. J Am Chem Soc, 2009, 131: 7542-7543

201 Zheng H, Smith RK, Jun Y, et al. Observation of single colloidal platinum nanocrystal growth trajectories. Science, 2009, 324: 1309-1312

202 Zhang HT, Ding J, Chow GM. Morphological control of synthesis and anomalous magnetic properties of 3-D branched Pt nanoparticles. Langmuir, 2008, 24: 375-378

203 Mazumdar D, Nagraj N, Kim HK, et al. Activity, folding and Z-DNA formation of the 8-17 DNAzyme in the presence of monovalent ions. J Am Chem Soc, 2009, 131: 5506-5515

204 Lim B, Kobayashi H, Camargo PHC, et al. New insights into the growth mechanism and surface structure of palladium nanocrystals. Nano Res, 2010, 3: 180-188

205 Yin Y, Alivisatos AP. Colloidal nanocrystal synthesis and the organic-inorganic interface. Nature, 2005, 437: 664-670

206 Niu Z, Peng Q, Gong M, et al. Oleylamine-mediated shape evolution of palladium nanocrystals. Angew Chem, 2011, 123: 6439-6443

207 DeSantis CJ, Skrabalak SE. Core values: elucidating the role of seed structure in the synthesis of symmetrically branched nanocrystals. J Am Chem Soc, 2013, 135: 10-13

208 Jin M, Liu H, Zhang H, et al. Synthesis of Pd nanocrystals enclosed by $\{100\}$ facets and with sizes $<10 \mathrm{~nm}$ for application in CO oxidation. Nano Res, 2011, 4: 83-91

209 Sau TK, Murphy CJ. Room temperature, high-yield synthesis of multiple shapes of gold nanoparticles in aqueous solution. J Am Chem Soc, 2004, 126: 8648-8649

210 Zhao H, Yang J, Wang L, et al. Fabrication of a palladium nanoparticle/graphene nanosheet hybrid via sacrifice of a copper template and its application in catalytic oxidation of formic acid. Chem Commun, 2011, 47: 2014-2016

211 Zhong C, Liu J, Ni Z, et al. Shape-controlled synthesis of Pt-Ir nanocubes with preferential (100) orientation and their unusual enhanced electrocatalytic activities. Sci China Mater, 2014, 57: $13-25$

212 Xu B, Yang H, Zhou G, et al. Strong metal-support interaction in size-controlled monodisperse palladium-hematite nano-heterostructures during a liquid-solid heterogeneous catalysis. Sci China Mater, 2014, 57: 34-41

213 Wu J, Pan YT, Su D, et al. Ultrathin and stable AgAu alloy nanowires. Sci China Mater, 2015, 58: 595-602

214 Guo S, Wang E. Noble metal nanomaterials: controllable synthesis and application in fuel cells and analytical sensors. Nano Today, 2011, 6: 240-264

215 Zhang L, Niu W, Xu G. Synthesis and applications of noble metal nanocrystals with high-energy facets. Nano Today, 2012, 7: 586-605

216 Yang CW, Chanda K, Lin PH, et al. Fabrication of Au-Pd core-shell heterostructures with systematic shape evolution using octahedral nanocrystal cores and their catalytic activity. J Am Chem Soc, 2011, 133: $19993-20000$

217 Wu HL, Kuo CH, Huang MH. Seed-mediated synthesis of gold nanocrystals with systematic shape evolution from cubic to trisoctahedral and rhombic dodecahedral structures. Langmuir, 2010, 26: 12307-12313

218 Yu Y, Zhang Q, Liu B, et al. Synthesis of nanocrystals with variable high-index Pd facets through the controlled heteroepitaxial growth of trisoctahedral Au templates. J Am Chem Soc, 2010, 132 18258-18265

219 Jin M, Zhang H, Xie Z, et al. Palladium concave nanocubes with high-index facets and their enhanced catalytic properties. Angew Chem Int Ed, 2011, 50: 7850-7854

220 Li J, Zheng Y, Zeng J, et al. Controlling the size and morphology of Au@Pd core-shell nanocrystals by manipulating the kinetics of seeded growth. Chem Eur J, 2012, 18: 8150-8156

221 Sun Y. Controlled synthesis of colloidal silver nanoparticles in organic solutions: empirical rules for nucleation engineering. Chem Soc Rev, 2013, 42: 2497-2511

222 Kramer RM, Li C, Naik RR. Engineered protein cages for nanomaterial synthesis. J Am Chem Soc, 2004, 126: 13282-13286

223 Ling T, Wang JJ, Zhang H, et al. Freestanding ultrathin metallic nanosheets: materials, synthesis, and applications. Adv Mater, 2015, 27: 5396-5402

224 Zhang H, Jin M, Wang J, et al. Nanocrystals composed of alternating shells of Pd and Pt can be obtained by sequentially adding different precursors. J Am Chem Soc, 2011, 133: 10422-10425

225 Fan FR, Liu DY, Wu YF, et al. Epitaxial growth of heterogeneous metal nanocrystals: from gold nano-octahedra to palladium and silver nanocubes. J Am Chem Soc, 2008, 130: 6949-6951

226 Bauer E, van der Merwe JH. Structure and growth of crystalline superlattices: from monolayer to superlattice. Phys Rev B, 1986, 33: $3657-3671$

227 Xie S, Peng HC, Lu N, et al. Confining the nucleation and overgrowth of Rh to the $\{111\}$ facets of Pd nanocrystal seeds: the roles of capping agent and surface diffusion. J Am Chem Soc, 2013, 135: $16658-16667$

228 Xia X, Figueroa-Cosme L, Tao J, et al. Facile synthesis of iridium nanocrystals with well-controlled facets using seed-mediated growth. J Am Chem Soc, 2014, 136: 10878-10881

229 Lee H, Habas SE, Somorjai GA, et al. Localized Pd overgrowth on cubic Pt nanocrystals for enhanced electrocatalytic oxidation of formic acid. J Am Chem Soc, 2008, 130: 5406-5407

230 Wu Y, Cai S, Wang D, et al. Syntheses of water-soluble octahedral, truncated octahedral, and cubic Pt-Ni nanocrystals and their structure-activity study in model hydrogenation reactions. J Am Chem Soc, 2012, 134: 8975-8981

231 Habas SE, Lee H, Radmilovic V, et al. Shaping binary meta nanocrystals through epitaxial seeded growth. Nat Mater, 2007, 6: 692-697

232 Zhang J, Fang J. A general strategy for preparation of Pt 3d-transition metal (Co, Fe, Ni) nanocubes. J Am Chem Soc, 2009, 131: $18543-18547$

233 Wu J, Yang H. Synthesis and electrocatalytic oxygen reduction properties of truncated octahedral $\mathrm{Pt}_{3} \mathrm{Ni}$ nanoparticles. Nano Res, 2011, 4: 72-82

234 Huo Z, Tsung C, Huang W, et al. Sub-two nanometer single crystal Au nanowires. Nano Lett, 2008, 8: 2041-2044

235 Jang K, Kim HJ, Son SU. Low-temperature synthesis of ultrathin rhodium nanoplates via molecular orbital symmetry interaction between rhodium precursors. Chem Mater, 2010, 22: 1273-1275

$236 \mathrm{Mu}$ R, Fu Q, Xu H, et al. Synergetic effect of surface and subsurface $\mathrm{Ni}$ species at $\mathrm{Pt}-\mathrm{Ni}$ bimetallic catalysts for $\mathrm{CO}$ oxidation. J Am Chem Soc, 2011, 133: 1978-1986

237 Wang C, Chi M, Li D, et al. Design and synthesis of bimetallic electrocatalyst with multilayered Pt-skin surfaces. J Am Chem Soc, 2011, 133: 14396-14403

238 Chen C, Kang Y, Huo Z, et al. Highly crystalline multimetallic nanoframes with three-dimensional electrocatalytic surfaces. Science, 2014, 343: 1339-1343 
239 Yu W, Porosoff MD, Chen JG. Review of Pt-based bimetallic catalysis: from model surfaces to supported catalysts. Chem Rev, 2012, 112: $5780-5817$

240 Niu W, Zhang L, Xu G. Shape-controlled synthesis of single-crystalline palladium nanocrystals. ACS Nano, 2010, 4: 1987-1996

241 Wiley B, Herricks T, Sun Y, et al. Polyol synthesis of silver nanoparticles: use of chloride and oxygen to promote the formation of single-crystal, truncated cubes and tetrahedrons. Nano Lett, 2004, 4: 1733-1739

242 Seo D, Park JC, Song H. Polyhedral gold nanocrystals with $O_{h}$ symmetry: from octahedra to cubes. J Am Chem Soc, 2006, 128: 14863-14870

243 Chen Y, Gu X, Nie CG, et al. Shape controlled growth of gold nanoparticles by a solution synthesis. Chem Commun, 2005, 4181

244 Chen J, Lim B, Lee EP, et al. Shape-controlled synthesis of platinum nanocrystals for catalytic and electrocatalytic applications. Nano Today, 2009, 4: 81-95

245 Lim SI, Ojea-Jiménez I, Varon M, et al. Synthesis of platinum cubes, polypods, cuboctahedrons, and raspberries assisted by cobalt nanocrystals. Nano Lett, 2010, 10: 964-973

246 Jin M, He G, Zhang H, et al. Shape-controlled synthesis of copper nanocrystals in an aqueous solution with glucose as a reducing agent and hexadecylamine as a capping agent. Angew Chem Int Ed, 2011, 50: 10560-10564

247 Zhang H, Li W, Jin M, et al. Controlling the morphology of rhodium nanocrystals by manipulating the growth kinetics with a syringe pump. Nano Lett, 2011, 11: 898-903

248 Dumestre F. Superlattices of iron nanocubes synthesized from $\mathrm{Fe}\left[\mathrm{N}\left(\mathrm{SiMe}_{3}\right)_{2}\right]_{3}$. Science, 2004, 303: 821-823

249 Seo D, Yoo CI, Chung IS, et al. Shape adjustment between multiply twinned and single-crystalline polyhedral gold nanocrystals: decahedra, icosahedra, and truncated tetrahedra. J Phys Chem C, 2008, 112: 2469-2475

250 Chen S, Wang ZL, Ballato J, et al. Monopod, bipod, tripod, and tetrapod gold nanocrystals. J Am Chem Soc, 2003, 125: 16186-16187

251 Lacroix LM, Gatel C, Arenal R, et al. Tuning complex shapes in platinum nanoparticles: from cubic dendrites to fivefold stars. Angew Chem, 2012, 124: 4768-4772

252 Xiong Y, Cai H, Yin Y, et al. Synthesis and characterization of fivefold twinned nanorods and right bipyramids of palladium. Chem Phys Lett, 2007, 440: 273-278

253 Wiley BJ, Xiong Y, Li ZY, et al. Right bipyramids of silver: a new shape derived from single twinned seeds. Nano Lett, 2006, 6: 765-768

254 Gao Y, Jiang P, Song L, et al. Studies on silver nanodecahedrons synthesized by PVP-assisted $N, N$-dimethylformamide (DMF) reduction. J Cryst Growth, 2006, 289: 376-380

255 Zhang W, Liu Y, Cao R, et al. Synergy between crystal strain and surface energy in morphological evolution of five-fold-twinned silver crystals. J Am Chem Soc, 2008, 130: 15581-15588

256 Pietrobon B, McEachran M, Kitaev V. Synthesis of size-controlled faceted pentagonal silver nanorods with tunable plasmonic properties and self-assembly of these nanorods. ACS Nano, 2009, 3: 21-26

257 Skrabalak SE, Xia Y. Pushing nanocrystal synthesis toward nanomanufacturing. ACS Nano, 2009, 3: 10-15

258 Choo H, He B, Liew KY, et al. Morphology and control of Pd nanoparticles. J Mol Catalysis A-Chem, 2006, 244: 217-228

259 Yang Y, Matsubara S, Xiong L, et al. Solvothermal synthesis of multiple shapes of silver nanoparticles and their SERS properties. J Phys Chem C, 2007, 111: 9095-9104

260 Washio I, Xiong Y, Yin Y, et al. Reduction by the end groups of poly(vinyl pyrrolidone): a new and versatile route to the kinetically controlled synthesis of Ag triangular nanoplates. Adv Mater, 2006, 18: $1745-1749$

261 Chu HC, Kuo CH, Huang MH. Thermal aqueous solution approach for the synthesis of triangular and hexagonal gold nanoplates with three different size ranges. Inorg Chem, 2006, 45: 808-813

262 Wang CY, Lu MY, Chen HC, et al. Single-crystalline $\mathrm{Pb}$ nanowires grown by galvanic displacement reactions of $\mathrm{Pb}$ ions on zinc foils and their superconducting properties. J Phys Chem C, 2007, 111: 6215-6219

263 Li H, Liao S. Synthesis of flower-like Co microcrystals composed of Co nanoplates in water/ethanol mixed solvent. J Phys D-Appl Phys, 2008, 41: 065004

264 Leng Y, Li Y, Li X, et al. Improved magnetic anisotropy of monodispersed triangular nickel nanoplates. J Phys Chem C, 2007, 111: 6630-6633

265 Zhang J, Yang H, Fang J, et al. Synthesis and oxygen reduction activity of shape-controlled $\mathrm{Pt}_{3} \mathrm{Ni}$ nanopolyhedra. Nano Lett, 2010, 10: 638-644

266 Wu J, Gross A, Yang H. Shape and composition-controlled platinum alloy nanocrystals using carbon monoxide as reducing agent. Nano Lett, 2011, 11: 798-802

267 Yin AX, Min XQ, Zhang YW, et al. Shape-selective synthesis and facet-dependent enhanced electrocatalytic activity and durability of monodisperse sub-10 nm Pt-Pd tetrahedrons and cubes. J Am Chem Soc, 2011, 133: 3816-3819

268 Xu D, Liu Z, Yang H, et al. Solution-based evolution and enhanced methanol oxidation activity of monodisperse platinum-copper nanocubes. Angew Chem Int Ed, 2009, 48: 4217-4221

269 Wu J, Qi L, You H, et al. Icosahedral platinum alloy nanocrystals with enhanced electrocatalytic activities. J Am Chem Soc, 2012, 134: $11880-11883$

270 Wang C, Hou Y, Kim J, et al. A general strategy for synthesizing FePt nanowires and nanorods. Angew Chem Int Ed, 2007, 46: 6333-6335

271 Liu Q, Yan Z, Henderson NL, et al. Synthesis of CuPt nanorod catalysts with tunable lengths. J Am Chem Soc, 2009, 131: 5720-5721

272 Hong JW, Lee YW, Kim M, et al. One-pot synthesis and electrocatalytic activity of octapodal $\mathrm{Au}-\mathrm{Pd}$ nanoparticles. Chem Commun, 2011, 47: 2553-2555

273 Chou SW, Zhu CL, Neeleshwar S, et al. Controlled growth and magnetic property of FePt nanostructure: cuboctahedron, octapod, truncated cube, and cube. Chem Mater, 2009, 21: 4955-4961

274 Wang L, Yamauchi Y. Controlled aqueous solution synthesis of platinum-palladium alloy nanodendrites with various compositions using amphiphilic triblock copolymers. Chem Asian J, 2010, 5: $2493-2498$

275 Lee YW, Kim M, Kim Y, et al. Synthesis and electrocatalytic activity of $\mathrm{Au}-\mathrm{Pd}$ alloy nanodendrites for ethanol oxidation. J Phys Chem C, 2010, 114: 7689-7693

276 Hong X, Wang D, Yu R, et al. Ultrathin $\mathrm{Au}-\mathrm{Ag}$ bimetallic nanowires with Coulomb blockade effects. Chem Commun, 2011, 47: 5160-5162

277 Peng Z, You H, Yang H. Composition-dependent formation of platinum silver nanowires. ACS Nano, 2010, 4: 1501-1510

278 Zhang $\mathrm{H}$, Jin $\mathrm{M}$, Liu $\mathrm{H}$, et al. Facile synthesis of Pd-Pt alloy nanocages and their enhanced performance for preferential oxidation of CO in excess hydrogen. ACS Nano, 2011, 5: 8212-8222

279 Yin $\mathrm{AX}$, Min XQ, Zhu W, et al. $\mathrm{Pt}-\mathrm{Cu}$ and $\mathrm{Pt}-\mathrm{Pd}-\mathrm{Cu}$ concave nanocubes with high-index facets and superior electrocatalytic activity. Chem Eur J, 2012, 18: 777-782

280 Phan DT, Uddin ASMI, Chung GS. A large detectable-range, 
high-response and fast-response resistivity hydrogen sensor based on $\mathrm{Pt} / \mathrm{Pd}$ core-shell hybrid with graphene. Sensors Actuators B-Chem, 2015, 220: 962-967

281 Wang F, Sun LD, Feng W, et al. Heteroepitaxial growth of core-shell and core-multishell nanocrystals composed of palladium and gold. Small, 2010, 6: 2566-2575

282 Bhattarai N, Prozorov T. In situ STEM investigation of shape-controlled synthesis of Au-Pd core-shell nanocubes. Microsc Microanal, 2015, 21: 951-952

283 Jin $\mathrm{M}$, Zhang $\mathrm{H}$, Wang J, et al. Copper can still be epitaxially deposited on palladium nanocrystals to generate core-shell nanocubes despite their large lattice mismatch. ACS Nano, 2012, 6: 2566-2573

284 Narula CK, Yang X, Li C, et al. A pathway for the growth of core-shell Pt-Pd nanoparticles. J Phys Chem C, 2015, 119: 25114-25121

285 Zhang P, Hu Y, Li B, et al. Kinetically stabilized Pd@Pt core-shell octahedral nanoparticles with thin Pt layers for enhanced catalytic hydrogenation performance. ACS Catal, 2015, 5: 1335-1343

286 Jiang M, Lim B, Tao J, et al. Epitaxial overgrowth of platinum on palladium nanocrystals. Nanoscale, 2010, 2: 2406-2411

287 Kobayashi H, Lim B, Wang J, et al. Seed-mediated synthesis of Pd-Rh bimetallic nanodendrites. Chem Phys Lett, 2010, 494: 249-254

288 Wang L, Yamauchi Y. Autoprogrammed synthesis of triple-layeredAu@Pd@Pt core-shell nanoparticles consisting of a Au@Pd bimetallic core and nanoporous Pt shell. J Am Chem Soc, 2010, 132: $13636-13638$

289 Wang F, Li C, Sun LD, et al. Heteroepitaxial growth of high-indexfaceted palladium nanoshells and their catalytic performance. J Am Chem Soc, 2011, 133: 1106-1111

290 Kim D, Lee YW, Lee SB, et al. Convex polyhedral Au@Pd core-shell nanocrystals with high-index facets. Angew Chem Int Ed, 2012, 51: 159-163

291 Park K, Vaia RA. Synthesis of complex Au/Ag nanorods by controlled overgrowth. Adv Mater, 2008, 20: 3882-3886

292 Zhang H, Jin M, Wang J, et al. Synthesis of Pd-Pt bimetallic nanocrystals with a concave structure through a bromide-induced galvanic replacement reaction. J Am Chem Soc, 2011, 133: 6078-6089

293 Wu J, Li P, Pan YTF, et al. Surface lattice-engineered bimetallic nanoparticles and their catalytic properties. Chem Soc Rev, 2012, 41: 8066-8074

294 Liu H, Nosheen F, Wang X. Noble metal alloy complex nanostructures: controllable synthesis and their electrochemical property. Chem Soc Rev, 2015, 44: 3056-3078

295 Zhang H, Jin M, Xia Y. Enhancing the catalytic and electrocatalytic properties of Pt-based catalysts by forming bimetallic nanocrystals with Pd. Chem Soc Rev, 2012, 41: 8035-8049

296 Wang D, Li Y. Bimetallic nanocrystals: liquid-phase synthesis and catalytic applications. Adv Mater, 2011, 23: 1044-1060

297 Ferrando R, Jellinek J, Johnston RL. Nanoalloys: from theory to applications of alloy clusters and nanoparticles. Chem Rev, 2008, 108: 845-910

298 Wei Z, Sun J, Li Y, et al. Bimetallic catalysts for hydrogen generation. Chem Soc Rev, 2012, 41: 7994-8008

299 Sankar M, Dimitratos N, Miedziak PJ, et al. Designing bimetallic catalysts for a green and sustainable future. Chem Soc Rev, 2012, 41: 8099-8139

300 Zheng YR, Gao MR, Li HH, et al. Carbon-supported $\mathrm{PtCo}_{2} \mathrm{Ni}_{2}$ alloy with enhanced activity and stability for oxygen reduction. Sci China Mater, 2015, 58: 179-185
301 Wang X, Zhuang J, Peng Q, et al. A general strategy for nanocrystal synthesis. Nature, 2005, 437: 121-124

302 Li P, Peng Q, Li Y. Controlled synthesis and self-assembly of highly monodisperse $\mathrm{Ag}$ and $\mathrm{Ag}_{2} \mathrm{~S}$ nanocrystals. Chem Eur J, 2011, 17: 941-946

303 Zhao Z, Zhou Z, Bao J, et al. Octapod iron oxide nanoparticles as high-performance $\mathrm{T}_{2}$ contrast agents for magnetic resonance imaging. Nat Commun, 2013, 4: 2266

304 Feng Q, Wang W, Cheong WC, et al. Synthesis of palladium and palladium sulfide nanocrystals via thermolysis of a $\mathrm{Pd}$-thiolate cluster. Sci China Mater, 2015, 58: 936-943

305 Sun S. Recent advances in chemical synthesis, self-assembly, and applications of FePt nanoparticles. Adv Mater, 2006, 18: 393-403

306 Chen M, Liu JP, Sun S. One-step synthesis of FePt nanoparticles with tunable size. J Am Chem Soc, 2004, 126: 8394-8395

307 Chen M, Kim J, Liu JP, et al. Synthesis of FePt nanocubes and their oriented self-assembly. J Am Chem Soc, 2006, 128: 7132-7133

308 Robinson I, Zacchini S, Tung LD, et al. Synthesis and characterization of magnetic nanoalloys from bimetallic carbonyl clusters. Chem Mater, 2009, 21: 3021-3026

309 Cumberland SL, Hanif KM, Javier A, et al. Inorganic clusters as single-source precursors for preparation of $\mathrm{CdSe}, \mathrm{ZnSe}$, and CdSe/ZnS nanomaterials. Chem Mater, 2002, 14: 1576-1584

310 Larsen TH, Sigman M, Ghezelbash A, et al. Solventless synthesis of copper sulfide nanorods by thermolysis of a single source thiolatederived precursor. J Am Chem Soc, 2003, 125: 5638-5639

311 Sigman MB, Ghezelbash A, Hanrath T, et al. Solventless synthesis of monodisperse $\mathrm{Cu}_{2} \mathrm{~S}$ nanorods, nanodisks, and nanoplatelets. J Am Chem Soc, 2003, 125: 16050-16057

312 Murray CB, Norris DJ, Bawendi MG. Synthesis and characterization of nearly monodisperse $\mathrm{CdE}(\mathrm{E}=$ sulfur, selenium, tellurium) semiconductor nanocrystallites. J Am Chem Soc, 1993, 115: 8706-8715

313 Hines MA, Guyot-Sionnest P. Bright UV-blue luminescent colloidal ZnSe nanocrystals. J Phys Chem B, 1998, 102: 3655-3657

314 Norris DJ, Yao N, Charnock FT, et al. High-quality manganesedoped ZnSe nanocrystals. Nano Lett, 2001, 1: 3-7

315 Li LS, Pradhan N, Wang Y, et al. High quality ZnSe and ZnS nanocrystals formed by activating zinc carboxylate precursors. Nano Lett, 2004, 4: 2261-2264

316 Hines MA, Scholes GD. Colloidal PbS nanocrystals with size-tunable near-infrared emission: observation of post-synthesis self-narrowing of the particle size distribution. Adv Mater, 2003, 15: 1844-1849

317 Lipovskii A, Kolobkova E, Petrikov V, et al. Synthesis and characterization of PbSe quantum dots in phosphate glass. Appl Phys Lett, 1997, 71: 3406-3408

318 Pietryga JM, Schaller RD, Werder D, et al. Pushing the band gap envelope: mid-infrared emitting colloidal PbSe quantum dots. J Am Chem Soc, 2004, 126: 11752-11753

319 Urban JJ, Talapin DV, Shevchenko EV, et al. Self-assembly of PbTe quantum dots into nanocrystal superlattices and glassy films. J Am Chem Soc, 2006, 128: 3248-3255

320 Urban JJ, Talapin DV, Shevchenko EV, et al. Synergism in binary nanocrystal superlattices leads to enhanced p-type conductivity in self-assembled $\mathrm{PbTe} / \mathrm{Ag}_{2} \mathrm{Te}$ thin films. Nat Mater, 2007, 6: 115-121

321 Zhuang Z, Lu X, Peng Q, et al. A facile "dispersion-decomposition" route to metal sulfide nanocrystals. Chem Eur J, 2011, 17: 10445-10452

322 Koch CC. Top-down synthesis of nanostructured materials: mechanical and thermal processing methods. Rev Adv Mater Sci, 2003, 5: 91-99 
323 Cobley CM, Xia Y. Engineering the properties of metal nanostructures via galvanic replacement reactions. Mater Sci Eng-R-Rep, 2010, 70: 44-62

324 Fan HJ, Gösele U, Zacharias M. Formation of nanotubes and hollow nanoparticles based on kirkendall and diffusion processes: a review. Small, 2007, 3: 1660-1671

325 Lou XWD, Archer LA, Yang Z. Hollow micro-/nanostructures: synthesis and applications. Adv Mater, 2008, 20: 3987-4019

326 Sun Y. Shape-controlled synthesis of gold and silver nanoparticles. Science, 2002, 298: 2176-2179

327 Yin Y. Formation of hollow nanocrystals through the nanoscale Kirkendall effect. Science, 2004, 304: 711-714

328 Métraux GS, Cao YC, Jin R, et al. Triangular nanoframes made of gold and silver. Nano Lett, 2003, 3: 519-522

329 Sun Y, Xia Y. Mechanistic study on the replacement reaction between silver nanostructures and chloroauric acid in aqueous medium. J Am Chem Soc, 2004, 126: 3892-3901

330 Skrabalak SE, Chen J, Sun Y, et al. Gold nanocages: synthesis, properties, and applications. Acc Chem Res, 2008, 41: 1587-1595

331 Macdonald JE, Bar Sadan M, Houben L, et al. Hybrid nanoscale inorganic cages. Nat Mater, 2010, 9: 810-815

332 Peng Z, You H, Wu J, et al. Electrochemical synthesis and catalytic property of sub-10 nm platinum cubic nanoboxes. Nano Lett, 2010, 10: $1492-1496$

333 Au L, Chen Y, Zhou F, et al. Synthesis and optical properties of cubic gold nanoframes. Nano Res, 2008, 1: 441-449

334 Peng S, Sun S. Synthesis and characterization of monodisperse hollow $\mathrm{Fe}_{3} \mathrm{O}_{4}$ nanoparticles. Angew Chem, 2007, 119: 4233-4236

335 Cabot A, Puntes VF, Shevchenko E, et al. Vacancy coalescence during oxidation of iron nanoparticles. J Am Chem Soc, 2007, 129: 10358-10360

336 Cabot A, Ibáñez M, Guardia P, et al. Reaction regimes on the synthesis of hollow particles by the Kirkendall effect. J Am Chem Soc, 2009, 131: 11326-11328

337 Chen J, Wiley B, McLellan J, et al. Optical properties of Pd-Ag and $\mathrm{Pt}-\mathrm{Ag}$ nanoboxes synthesized via galvanic replacement reactions. Nano Lett, 2005, 5: 2058-2062

338 Wu X, Yu Y, Liu Y, et al. Synthesis of hollow $\mathrm{Cd}_{x} \mathrm{Zn}_{1-x} \mathrm{Se}$ nanoframes through the selective cation exchange of inorganic-organic hybrid $\mathrm{ZnSe}$-amine nanoflakes with cadmium ions. Angew Chem, 2012, 124: 3265-3269

339 Kottmann JP, Martin OJF, Smith DR, et al. Plasmon resonances of silver nanowires with a nonregular cross section. Phys Rev B, 2001, 64: 235402

340 Moffatt WG. The Handbook of Binary Phase Diagrams. Amsterdam: Genium Pub Corp, 1976

341 Zhang Q, Cobley CM, Zeng J, et al. Dissolving Ag from Au-Ag alloy nanoboxes with $\mathrm{H}_{2} \mathrm{O}_{2}$ : a method for both tailoring the optical properties and measuring the $\mathrm{H}_{2} \mathrm{O}_{2}$ concentration. J Phys Chem C, 2010, 114: 6396-6400

342 Popa A, Samia AC. Effect of metal precursor on the growth and electrochemical sensing properties of Pt-Ag nanoboxes. Chem Comm, 2014, 55: 7295-7298

343 Chen J, Wiley B, Li ZY, et al. Gold nanocages: engineering their structure for biomedical applications. Adv Mater, 2005, 17: 2255-2261

344 Gonzalez E, Arbiol J, Puntes VF. Carving at the nanoscale: sequential galvanic exchange and kirkendall growth at room temperature. Science, 2011, 334: 1377-1380

$345 \mathrm{Oh} \mathrm{MH,} \mathrm{Yu} \mathrm{T,} \mathrm{Yu} \mathrm{SH,} \mathrm{et} \mathrm{al.} \mathrm{Galvanic} \mathrm{replacement} \mathrm{reactions} \mathrm{in} \mathrm{metal}$ oxide nanocrystals. Science, 2013, 340: 964-968

346 Wang ZL, Ahmad TS, El-Sayed MA. Steps, ledges and kinks on the surfaces of platinum nanoparticles of different shapes. Surface Sci, 1997, 380: 302-310

347 Hong X, Wang D, Cai S, et al. Single-crystalline octahedral Au-Ag nanoframes. J Am Chem Soc, 2012, 134: 18165-18168

348 Fetisov VB, Kozhina GA, Ermakov AN, et al. Electrochemical dissolution of $\mathrm{Mn}_{3} \mathrm{O}_{4}$ in acid solutions. J Solid State Electrochem, 2007, 11: 1205-1210

349 Villinski JE, O'Day PA, Corley TL, et al. In situ spectroscopic and solution analyses of the reductive dissolution of $\mathrm{MnO}_{2}$ by $\mathrm{Fe}(\mathrm{II})$. Environ Sci Technol, 2001, 35: 1157-1163

350 Sun Y, Mayers B, Xia Y. Metal nanostructures with hollow interiors. Adv Mater, 2003, 15: 641-646

351 Sun Y, Tao Z, Chen J, et al. Ag nanowires coated with Ag/Pd alloy sheaths and their use as substrates for reversible absorption and desorption of hydrogen. J Am Chem Soc, 2004, 126: 5940-5941

$352 \mathrm{Hu} \mathrm{M}$, Petrova H, Chen J, et al. Ultrafast laser studies of the photothermal properties of gold nanocages. J Phys Chem B, 2006, 110: 1520-1524

353 Wan DH, Xia XH, Xia YN, et al. Robust synthesis of gold cubic nanoframes through a combination of galvanic replacement, gold deposition, and silver dealloying. Small, 2013, 9: 3111-3117

354 Cobley CM, Campbell DJ, Xia Y. Tailoring the optical and catalytic properties of gold-silver nanoboxes and nanocages by introducing palladium. Adv Mater, 2008, 20: 748-752

355 Sun Y, Wiley B, Li ZY, et al. Synthesis and optical properties of nanorattles and multiple-walled nanoshells/nanotubes made of metal alloys. J Am Chem Soc, 2004, 126: 9399-9406

356 Tan Q, Wang $\mathrm{P}$, Liu $\mathrm{H}$, et al. Hollow $\mathrm{MO}_{x}-\mathrm{RuO}_{2}(\mathrm{M}=\mathrm{Co}, \mathrm{Cu}, \mathrm{Fe}$, $\mathrm{Ni}, \mathrm{CuNi}$ ) nanostructures as highly efficient electrodes for supercapacitors. Sci China Mater, 2016, 59: 323-336

357 Lieber CM, Hu J, Ouyang M, et al. Controlled growth and electrical properties of heterojunctions of carbon nanotubes and silicon nanowires. Nature, 1999, 399: 48-51

358 Xiang J, Lu W, Hu Y, et al. Ge/Si nanowire heterostructures as highperformance field-effect transistors. Nature, 2006, 441: 489-493

359 Hsieh CH, Chou LJ, Lin GR, et al. Nanophotonic switch: gold-in$\mathrm{Ga}_{2} \mathrm{O}_{3}$ peapod nanowires. Nano Lett, 2008, 8: 3081-3085

360 Tao F, Grass ME, Zhang Y, et al. Reaction-driven restructuring of Rh-Pd and Pt-Pd core-shell nanoparticles. Science, 2008, 322: 932-934

361 Elmalem E, Saunders AE, Costi R, et al. Growth of photocatalytic CdSe-Pt nanorods and nanonets. Adv Mater, 2008, 20: 4312-4317

362 Teng X, Feygenson M, Wang Q, et al. Electronic and magnetic properties of ultrathin $\mathrm{Au} / \mathrm{Pt}$ nanowires. Nano Lett, 2009, 9: 3177-3184

363 Wen CY, Reuter MC, Bruley J, et al. Formation of compositionally abrupt axial heterojunctions in silicon-germanium nanowires. Science, 2009, 326: 1247-1250

364 Oka K, Yanagida T, Nagashima K, et al. Resistive-switching memory effects of $\mathrm{NiO}$ nanowire/metal junctions. J Am Chem Soc, 2010, 132: 6634-6635

365 Kim KW, Kim SM, Choi S, et al. Electroless Pt deposition on $\mathrm{Mn}_{3} \mathrm{O}_{4}$ nanoparticles via the galvanic replacement process: electrocatalytic nanocomposite with enhanced performance for oxygen reduction reaction. ACS Nano, 2012, 6: 5122-5129

366 Xiong L, Li S, Zhang B, et al. Galvanic replacement-mediated synthesis of hollow $\mathrm{Cu}_{2} \mathrm{O}-\mathrm{Au}$ nanocomposites and $\mathrm{Au}$ nanocages for catalytic and SERS applications. RSC Adv, 2015, 5: 76101-76106

367 Smith DJ, Petford-long AK, Wallenberg LR, et al. Dynamic atomiclevel rearrangements in small gold particles. Science, 1986, 233: $872-875$ 
ang Y, Xie S, Liu J, et al. Shape-controlled synthesis of palladium nanocrystals: a mechanistic understanding of the evolution from octahedrons to tetrahedrons. Nano Lett, 2013, 13: 2276-2281

369 Xiong Y, Xia Y. Shape-controlled synthesis of metal nanostructures: the case of palladium. Adv Mater, 2007, 19: 3385-3391

370 Xiong Y, Chen J, Wiley B, et al. Understanding the role of oxidative etching in the polyol synthesis of Pd nanoparticles with uniform shape and size. J Am Chem Soc, 2005, 127: 7332-7333

371 Cobley CM, Rycenga M, Zhou F, et al. Etching and growth: an intertwined pathway to silver nanocrystals with exotic shapes. Angew Chem Int Ed, 2009, 48: 4824-4827

372 Guo X, Zhang Q, Sun Y, et al. Lateral etching of core-shell Au@metal nanorods to metal-tipped Au nanorods with improved catalytic activity. ACS Nano, 2012, 6: 1165-1175

373 Huang CC, Hwu JR, Su WC, et al. Surfactant-assisted hollowing of $\mathrm{Cu}$ nanoparticles involving halide-induced corrosion-oxidation processes. Chem Eur J, 2006, 12: 3805-3810

374 Kisner A, Heggen M, Fernández E, et al. The role of oxidative etching in the synthesis of ultrathin single-crystalline Au nanowires. Chem Eur J, 2011, 17: 9503-9507

375 Xia X, Choi SI, Herron JA, et al. Facile synthesis of palladium right bipyramids and their use as seeds for overgrowth and as catalysts for formic acid oxidation. J Am Chem Soc, 2013, 135: 15706-15709 nanocubes and their conversion to $\mathrm{Rh}$ cubic nanoframes by selective etching of the Pd cores. Angew Chem Int Ed, 2012, 51: 10266-10270

377 Thurmer K. Autocatalytic oxidation of lead crystallite surfaces. Science, 2002, 297: 2033-2035

378 Ma L, Wang C, Gong M, et al. Control over the branched structures of platinum nanocrystals for electrocatalytic applications. ACS Nano, 2012, 6: 9797-9806

379 Im SH, Lee YT, Wiley B, et al. Large-scale synthesis of silver nanocubes: the role of $\mathrm{HCl}$ in promoting cube perfection and monodispersity. Angew Chem Int Ed, 2005, 117: 2192-2195

380 Wulff G. XXV. Zur Frage der Geschwindigkeit des Wachsthums und der Auflösung der Krystallflächen. Zeitschrift für Kristallographie-Crystal Mater, 1901, 34: 449-530

381 Marks LD. Experimental studies of small particle structures. Rep Prog Phys, 1994, 57: 603-649

382 Mettela G, Kulkarni GU. Facet selective etching of Au microcrystallites. Nano Res, 2015, 8: 2925-2934

383 Wu Y, Wang D, Niu Z, et al. A strategy for designing a concave Pt-Ni alloy through controllable chemical etching. Angew Chem Int Ed, 2012, 51: 12524-12528

384 Xiong Y, Wiley B, Chen J, et al. Corrosion-based synthesis of single-crystal Pd nanoboxes and nanocages and their surface plasmon properties. Angew Chem Int Ed, 2005, 44: 7913-7917

$385 \mathrm{Lu} \mathrm{X}, \mathrm{Au} \mathrm{L}, \mathrm{McL}$ llan J, et al. Fabrication of cubic nanocages and nanoframes by dealloying $\mathrm{Au} / \mathrm{Ag}$ alloy nanoboxes with an aqueous etchant based on $\mathrm{Fe}\left(\mathrm{NO}_{3}\right)_{3}$ or $\mathrm{NH}_{4} \mathrm{OH}$. Nano Lett, 2007, 7: 1764-1769

386 Li B, Long R, Zhong X, et al. Investigation of size-dependent plasmonic and catalytic properties of metallic nanocrystals enabled by size control with $\mathrm{HCl}$ oxidative etching. Small, 2012, 8: 1710-1716

387 Wiley BJ, Chen Y, McLellan JM, et al. Synthesis and optical properties of silver nanobars and nanorice. Nano Lett, 2007, 7: 1032-1036

388 Teng X, Han WQ, Ku W, et al. Synthesis of ultrathin palladium and platinum nanowires and a study of their magnetic properties. Angew Chem, 2008, 120: 2085-2088

389 Korte KE, Skrabalak SE, Xia Y. Rapid synthesis of silver nanowires through a CuCl- or $\mathrm{CuCl}_{2}$-mediated polyol process. J Mater Chem,
2008, 18: 437-441

390 Miszta K, Dorfs D, Genovese A, et al. Cation exchange reactions in colloidal branched nanocrystals. ACS Nano, 2011, 5: 7176-7183

391 Casavola M, van Huis MA, Bals S, et al. Anisotropic cation exchange in $\mathrm{PbSe} / \mathrm{CdSe}$ core/shell nanocrystals of different geometry. Chem Mater, 2012, 24: 294-302

392 Son DH, Hughes SM, Yin YD, et al. Cation exchange rea ctions in ioni cnanocrys tals. Science, 2004, 306: 1009-1012

393 Mocatta D, Cohen G, Schattner J, et al. Heavily doped semiconductor nanocrystal quantum dots. Science, 2011, 332: 77-81

394 Chan EM, Marcus MA, Fakra S, et al. Millisecond kinetics of nanocrystal cation exchange using microfluidic X-ray absorption spectroscopy. J Phys Chem A, 2007, 111: 12210-12215

395 Plendl JN, Gielisse PJ. Atomistic expression of hardness. Zeitschrift für Kristallographie, 1963, 118: 404-421

396 Marcus Y. Thermodynamics of solvation of ions. Part 5.-Gibbs free energy of hydration at 298.15 K. J Chem Soc Faraday Trans, 1991, 87: 2995-2999

397 Wang F, Sun LD, Gu J, et al. Selective heteroepitaxial nanocrystal growth of rare earth fluorides on sodium chloride: synthesis and density functional calculations. Angew Chem Int Ed, 2012, 51: 8796-8799

398 Kim S, Fisher B, Eisler HJ, et al. Type-II quantum dots: $\mathrm{CdTe} / \mathrm{CdSe}$ (core/shell) and $\mathrm{CdSe} / \mathrm{ZnTe}$ (core/shell) heterostructures. J Am Chem Soc, 2003, 125: 11466-11467

399 Peng X, Schlamp MC, Kadavanich AV, et al. Epitaxial growth of highly luminescent $\mathrm{CdSe} / \mathrm{CdS}$ core/shell nanocrystals with photostability and electronic accessibility. J Am Chem Soc, 1997, 119: 7019-7029

400 Sitt A, Sala FD, Menagen G, et al. Multiexciton engineering in seeded core/shell nanorods: transfer from type-I to quasi-type-II regimes. Nano Lett, 2009, 9: 3470-3476

401 Talapin DV, Nelson JH, Shevchenko EV, et al. Seeded growth of highly luminescent $\mathrm{CdSe} / \mathrm{CdS}$ nanoheterostructures with rod and tetrapod morphologies. Nano Lett, 2007, 7: 2951-2959

402 Lambert K, Geyter BD, Moreels I, et al. PbTe|CdTe core|shell particles by cation exchange, a HR-TEM study. Chem Mater, 2009, 21: 778-780

403 Pietryga JM, Werder DJ, Williams DJ, et al. Utilizing the lability of lead selenide to produce heterostructured nanocrystals with bright, stable infrared emission. J Am Chem Soc, 2008, 130: 4879-4885

404 Miszta K, de Graaf J, Bertoni G, et al. Hierarchical self-assembly of suspended branched colloidal nanocrystals into superlattice structures. Nat Mater, 2011, 10: 872-876

405 Sadtler B, Demchenko DO, Zheng H, et al. Selective facet reactivity during cation exchange in cadmium sulfide nanorods. J Am Chem Soc, 2009, 131: 5285-5293

406 Liu J, Zhao Y, Liu J, et al. From $\mathrm{Cu}_{2} \mathrm{~S}$ nanocrystals to $\mathrm{Cu}$ doped $\mathrm{CdS}$ nanocrystals through cation exchange: controlled synthesis, optical properties and their p-type conductivity research. Sci China Mater, 2015, 58: 693-703

407 Luther JM, Zheng H, Sadtler B, et al. Synthesis of $\mathrm{PbS}$ nanorods and other ionic nanocrystals of complex morphology by sequential cation exchange reactions. J Am Chem Soc, 2009, 131: 16851-16857

408 Park J, Kim SW. CuInS2/ZnS core/shell quantum dots by cation exchange and their blue-shifted photoluminescence. J Mater Chem, 2011, 21: 3745

409 Mao B, Chuang CH, Lu F, et al. Study of the partial Ag-to-Zn cation exchange in $\mathrm{AgInS}_{2} / \mathrm{ZnS}$ nanocrystals. J Phys Chem C, 2013, 117: 648-656

410 Zhang B, Jung Y, Chung HS, et al. Nanowire transformation by 
size-dependent cation exchange reactions. Nano Lett, 2010, 10: $149-155$

411 Li X, Zhang G, Bai X, et al. Highly conducting graphene sheets and Langmuir-Blodgett films. Nat Nanotech, 2008, 3: 538-542

412 Novoselov KS. Electric field effect in atomically thin carbon films. Science, 2004, 306: 666-669

413 Novoselov KS, Jiang D, Schedin F, et al. Two-dimensional atomic crystals. Proc Natl Acad Scis, 2005, 102: 10451-10453

414 Berger C. Electronic confinement and coherence in patterned epitaxial graphene. Science, 2006, 312: 1191-1196

415 Berger C, Song Z, Li T, et al. Ultrathin epitaxial graphite: 2D electron gas properties and a route toward graphene-based nanoelectronics. J Phys Chem B, 2004, 108: 19912-19916

416 Greinke RA, Reyolds III RA. Expandable graphite and method, United States Patent Application, 2002

417 Hernandez Y, Nicolosi V, Lotya M, et al. High-yield production of graphene by liquid-phase exfoliation of graphite. Nat Nanotech, 2008, 3: 563-568

418 Zeng Z, Yin Z, Huang X, et al. Single-layer semiconducting nanosheets: high-yield preparation and device fabrication. Angew Chem Int Ed, 2011, 50: 11093-11097

419 Coleman JN, Lotya M, O'Neill A, et al. Two-dimensional nanosheets produced by liquid exfoliation of layered materials. Science, 2011, 331: 568-571

420 Eswaraiah V, Jyothirmayee Aravind SS, Ramaprabhu S. Top down method for synthesis of highly conducting graphene by exfoliation of graphite oxide using focused solar radiation. J Mater Chem, 2011, 21: 6800-6803

421 Zeng Z, Sun T, Zhu J, et al. An effective method for the fabrication of few-layer-thick inorganic nanosheets. Angew Chem Int Ed, 2012, 51: 9052-9056

422 Adachi-Pagano M, Forano C, Besse JP. Delamination of layered double hydroxides by use of surfactants. Chem Commun, 2000, 91-92

423 O'Leary S, O'Hare D, Seeley G. Delamination of layered double hydroxides in polar monomers: new LDH-acrylate nanocompositesElectronic supplementary information (ESI) available: TEM image of $\mathrm{Mg}_{2} \mathrm{Al}(\mathrm{Cl})$ showing the layered structure. Chem Commun, 2002, 1506-1507

424 Jobbágy M, Regazzoni AE. Delamination and restacking of hybrid layered double hydroxides assessed by in situ XRD. J Colloid Interface Sci, 2004, 275: 345-348

425 Ma R, Liu Z, Li L, et al. Exfoliating layered double hydroxides in formamide: a method to obtain positively charged nanosheets. J Mater Chem, 2006, 16: 3809-3813

426 Liu Z, Ma R, Osada M, et al. Synthesis, anion exchange, and delamination of $\mathrm{Co}-\mathrm{Al}$ layered double hydroxide: assembly of the exfoliated nanosheet/polyanion composite films and magneto-optical studies. J Am Chem Soc, 2006, 128: 4872-4880

427 Li L, Ma R, Ebina Y, et al. Positively charged nanosheets derived via total delamination of layered double hydroxides. Chem Mater, 2005, 17: 4386-4391

428 Zhang J, Sasaki K, Sutter E, et al. Stabilization of platinum oxygenreduction electrocatalysts using gold clusters. Science, 2007, 315: 220-222

429 Li G, Kobayashi H, Taylor JM, et al. Hydrogen storage in Pd nanocrystals covered with a metal-organic framework. Nat Mater, 2014, 13: 802-806

430 Kuo CH, Tang Y, Chou LY, et al. Yolk-shell nanocrystal@ZIF-8 nanostructures for gas-phase heterogeneous catalysis with selectivity control. J Am Chem Soc, 2012, 134: 14345-14348

431 Fu Q, Li WX, Yao Y, et al. Interface-confined ferrous centers for catalytic oxidation. Science, 2010, 328: 1141-1144

432 Chen G, Xu C, Huang X, et al. Interfacial electronic effects control the reaction selectivity of platinum catalysts. Nat Mater, 2016, 15: 564-569

433 Kim F, Connor S, Song H, et al. Platonic gold nanocrystals. Angew Chem, 2004, 116: 3759-3763

434 Li C, Fan F, Yin B, et al. $\mathrm{Au}^{+}$-cetyltrimethylammonium bromide solution: a novel precursor for seed-mediated growth of gold nanoparticles in aqueous solution. Nano Res, 2013, 6: 29-37

435 Lim B, Jiang M, Yu T, et al. Nucleation and growth mechanisms for Pd-Pt bimetallic nanodendrites and their electrocatalytic properties. Nano Res, 2010, 3: 69-80

436 Johnson NJJ, van Veggel FCJM. Sodium lanthanide fluoride coreshell nanocrystals: a general perspective on epitaxial shell growth. Nano Res, 2013, 6: 547-561

437 Zhao Y, de la Mata M, Qiu RLJ, et al. Te-seeded growth of fewquintuple layer $\mathrm{Bi}_{2} \mathrm{Te}_{3}$ nanoplates. Nano Res, 2014, 7: 1243-1253

438 Hillerich K, Dick KA, Messing ME, et al. Simultaneous growth mechanisms for $\mathrm{Cu}$-seeded InP nanowires. Nano Res, 2012, 5: 297-306

439 Li C, Wei R, Xu Y, et al. Synthesis of hexagonal and triangular $\mathrm{Fe}_{3} \mathrm{O}_{4}$ nanosheets via seed-mediated solvothermal growth. Nano Res, 2014, 7: 536-543

440 Gole A, Murphy CJ. seed-mediated synthesis of gold nanorods: role of the size and nature of the seed. Chem Mater, 2004, 16: 3633-3640

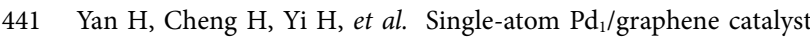
achieved by atomic layer deposition: remarkable performance in selective hydrogenation of 1,3-butadiene. J Am Chem Soc, 2015, 137: 10484-10487

442 Whitesides GM. Self-assembly at all scales. Science, 2002, 295: 2418-2421

443 Bishop KJM, Wilmer CE, Soh S, et al. Nanoscale forces and their uses in self-assembly. Small, 2009, 5: 1600-1630

444 Grzelczak M, Vermant J, Furst EM, et al. Directed self-assembly of nanoparticles. ACS Nano, 2010, 4: 3591-3605

445 Min Y, Akbulut M, Kristiansen K, et al. The role of interparticle and external forces in nanoparticle assembly. Nat Mater, 2008, 7: $527-538$

$446 \mathrm{Wu}$ S, Ang CY, Luo Z, et al. Byproduct-induced in-situ formation of gold colloidal superparticles. Sci China Mater, 2015, 58: 860-866

447 Wang C, Siu C, Zhang J, et al. Understanding the forces acting in self-assembly and the implications for constructing three-dimensional (3D) supercrystals. Nano Res, 2015, 8: 2445-2466

448 Ozin GA, Arsenault A, Cademartiri L. Nanochemistry: A Chemical Approach to Nanomaterials. London: RSC Publishing, 2009

449 Cademartiri L, Ozin GA. Concepts of Nanochemistry. Berlin: John Wiley \& Sons, 2009

450 Murray CB, Kagan CR, Bawendi MG. Self-organization of CdSe nanocrystallites into three-dimensional quantum dot superlattices. Science, 1995, 270: 1335-1338

451 Redl FX, Cho KS, Murray CB, et al. Three-dimensional binary superlattices of magnetic nanocrystals and semiconductor quantum dots. Nature, 2003, 423: 968-971

452 Shevchenko EV, Talapin DV, Kotov NA, et al. Structural diversity in binary nanoparticle superlattices. Nature, 2006, 439: 55-59

453 Talapin DV, Shevchenko EV, Bodnarchuk MI, et al. Quasicrystalline order in self-assembled binary nanoparticle superlattices. Nature, 2009, 461: 964-967

454 Bai F, Wang D, Huo Z, et al. A versatile bottom-up assembly approach to colloidal spheres from nanocrystals. Angew Chem Int Ed, 2007, 46: 6650-6653 
ang $\mathrm{P}, \mathrm{Yu} \mathrm{Q}$, Long $\mathrm{Y}$, et al. Multivalent assembly of ultrasmal nanoparticles: one-, two-, and three-dimensional architectures of 2 nm gold nanoparticles. Nano Res, 2012, 5: 283-291

456 Hong X, Tan C, Liu J, et al. AuAg nanosheets assembled from ultrathin AuAg nanowires. J Am Chem Soc, 2015, 137: 1444-1447

457 Sun X, Zhu X, Zhang N, et al. Controlling and self assembling of monodisperse platinum nanocubes as efficient methanol oxidation electrocatalysts. Chem Commun, 2015, 51: 3529-3532

458 Zhang H, Wang D. Controlling the growth of charged-nanoparticle chains through interparticle electrostatic repulsion. Angew Chem, 2008, 120: 4048-4051

459 Hu C, Lin K, Wang X, et al. Electrostatic self-assembling formation of Pd superlattice nanowires from surfactant-free ultrathin Pd nanosheets. J Am Chem Soc, 2014, 136: 12856-12859

460 Fu G, Zhang Q, Wu J, et al. Arginine-mediated synthesis of cubelike platinum nanoassemblies as efficient electrocatalysts. Nano Res, 2015, 8: 3963-3971

461 Tan C, Qi X, Liu Z, et al. Self-assembled chiral nanofibers from ultrathin low-dimensional nanomaterials. J Am Chem Soc, 2015, 137: 1565-1571

462 Fu Q, Ran G, Xu W. Direct self-assembly of CTAB-capped Au nanotriangles. Nano Res, 2016

463 Zhang SY, Regulacio MD, Han MY. Self-assembly of colloidal onedimensional nanocrystals. Chem Soc Rev, 2014, 43: 2301-2323

464 Wang T, Zhuang J, Lynch J, et al. Self-assembled colloidal superparticles from nanorods. Science, 2012, 338: 358-363

465 Macfarlane RJ, Lee B, Jones MR, et al. Nanoparticle superlattice engineering with DNA. Science, 2011, 334: 204-208

466 Biancaniello PL, Kim AJ, Crocker JC. Colloidal interactions and self-assembly using DNA hybridization. Phys Rev Lett, 2005, 94 058302

467 Nykypanchuk D, Maye MM, van der Lelie D, et al. DNA-guided crystallization of colloidal nanoparticles. Nature, 2008, 451: 549-552

468 Park SY, Lytton-Jean AKR, Lee B, et al. DNA-programmable nanoparticle crystallization. Nature, 2008, 451: 553-556

469 Leunissen ME, Christova CG, Hynninen AP, et al. Ionic colloidal crystals of oppositely charged particles. Nature, 2005, 437: 235-240

470 Feng L, Dreyfus R, Sha R, et al. DNA patchy particles. Adv Mater, 2013, 25: 2779-2783

471 Yan W, Xu L, Xu C, et al. Self-assembly of chiral nanoparticle pyramids with strong $R / S$ optical activity. J Am Chem Soc, 2012, 134: 15114-15121

472 Wang Y, Wang Y, Breed DR, et al. Colloids with valence and specific directional bonding. Nature, 2012, 491: 51-55

473 Chen Q, Whitmer JK, Jiang S, et al. Supracolloidal reaction kinetics of janus spheres. Science, 2011, 331: 199-202

474 Nie Z, Fava D, Kumacheva E, et al. Self-assembly of metal-polymer analogues of amphiphilic triblock copolymers. Nat Mater, 2007, 6 : 609-614

475 Liu K, Nie Z, Zhao N, et al. Step-growth polymerization of inorganic nanoparticles. Science, 2010, 329: 197-200

476 Cademartiri L, Bishop KJM. Programmable self-assembly. Nat Mater, 2014, 14: 2-9

477 Quan Z, Fang J. Superlattices with non-spherical building blocks. Nano Today, 2010, 5: 390-411

478 Li F, Josephson DP, Stein A. Colloidal assembly: the road from particles to colloidal molecules and crystals. Angew Chem Int Ed, 2011, 50: 360-388

479 Ciszek JW, Huang L, Tsonchev S, et al. Assembly of nanorods into designer superstructures: the role of templating, capillary forces, adhesion, and polymer hydration. ACS Nano, 2010, 4: 259-266

480 Stebe KJ, Lewandowski E, Ghosh M. Oriented assembly of metamaterials. Science, 2009, 325: 159-160

481 Evers WH, Nijs BD, Filion L, et al. Entropy-driven formation of binary semiconductor-nanocrystal superlattices. Nano Lett, 2010, 10: 4235-4241

482 Bodnarchuk MI, Kovalenko MV, Heiss W, et al. Energetic and entropic contributions to self-assembly of binary nanocrystal superlattices: temperature as the structure-directing factor. J Am Chem Soc, 2010, 132: 11967-11977

483 Blaak R, Mulder BM, Frenkel D. Cubatic phase for tetrapods. J Chem Phys, 2004, 120: 5486-5492

484 Parviz BA, Ryan D, Whitesides GM. Using self-assembly for the fabrication of nano-scale electronic and photonic devices. IEEE Trans Adv Packag, 2003, 26: 233-241

485 Pileni MP. Nanocrystal self-assemblies: fabrication and collective properties. J Phys Chem B, 2001, 105: 3358-3371

486 Palmer LC, Stupp SI. Molecular self-assembly into one-dimensional nanostructures. Acc Chem Res, 2008, 41: 1674-1684

487 Vos WL, Sprik R, van Blaaderen A, et al. Strong effects of photonic band structures on the diffraction of colloidal crystals. Phys Rev B, 1996, 53: 16231-16235

488 Li J, Huang W, Wang Z, et al. A reversibly tunable colloidal photonic crystal via the infiltrated solvent liquid-solid phase transition. Colloid Surf A-Physicochem Eng Asp, 2007, 293: 130-134

489 Shimoda Y, Ozaki M, Yoshino K. Electric field tuning of a stop band in a reflection spectrum of synthetic opal infiltrated with nematic liquid crystal. Appl Phys Lett, 2001, 79: 3627-3629

490 Kim H, Ge J, Kim J, et al. Structural colour printing using a magnetically tunable and lithographically fixable photonic crystal. Nat Photon, 2009, 3: 534-540

491 Jiao Y, Han D, Liu L, et al. Highly ordered mesoporous few-layer graphene frameworks enabled by $\mathrm{Fe}_{3} \mathrm{O}_{4}$ nanocrystal superlattices. Angew Chem, 2015, 127: 5819-5823

492 Chen W, Yu R, Li L, et al. A seed-based diffusion route to monodisperse intermetallic CuAu nanocrystals. Angew Chim, 2010, 122: 2979-2983

493 Wang D, Li Y. One-pot protocol for Au-based hybrid magnetic nanostructures via a noble-metal-induced reduction process. J Am Chem Soc, 2010, 132: 6280-6281

494 Yu R, Chen W, Cheng ZY, et al. Multishell intermetallic onions by symmetrical configuration of ordered domains. Phys Rev Lett, 2010, 105: 225501

495 Carenco S, Portehault D, Boissière C, et al. Nanoscaled meta borides and phosphides: recent developments and perspectives. Chem Rev, 2013, 113: 7981-8065

496 Wang D, Li Y. Controllable synthesis of Cu-based nanocrystals in ODA solvent. Chem Commun, 2011, 47: 3604-3606

497 Jia X, Zhao Y, Chen G, et al. $\mathrm{Ni}_{3} \mathrm{FeN}$ znanoparticles derived from ultrathin NiFe-layered double hydroxide nanosheets: an efficient overall water splitting electrocatalyst. Adv Energy Mater, 2016, 6: 1502585

$498 \mathrm{Bi} \mathrm{W}, \mathrm{Hu} \mathrm{Z}, \mathrm{Li}$ X, et al. Metallic mesocrystal nanosheets of vanadium nitride for high-performance all-solid-state pseudocapacitors. Nano Res, 2015, 8: 193-200

$499 \mathrm{Xu} \mathrm{K}$, Chen $\mathrm{P}, \mathrm{Li}$ X, et al. Metallic nickel nitride nanosheets realizing enhanced electrochemical water oxidation. J Am Chem Soc, 2015, 137: 4119-4125

500 Lee BS, Yi M, Chu SY, et al. Copper nitride nanoparticles supported on a superparamagnetic mesoporous microsphere for toxicfree click chemistry. Chem Commun, 2010, 46: 3935-3937

501 Tian J, Liu Q, Asiri AM, et al. Self-supported nanoporous cobalt 
phosphide nanowire arrays: an efficient 3D hydrogen-evolving cathode over the wide range of $\mathrm{pH}$ 0-14. J Am Chem Soc, 2014, 136: $7587-7590$

502 Jiang K, Xu K, Zou S, et al. B-doped Pd catalyst: boosting roomtemperature hydrogen production from formic acid-formate solutions. J Am Chem Soc, 2014, 136: 4861-4864

503 Popczun EJ, Read CG, Roske CW, et al. Highly active electrocatalysis of the hydrogen evolution reaction by cobalt phosphide nanoparticles. Angew Chem, 2014, 126: 5531-5534

504 Niu Z, Wang D, Yu R, et al. Highly branched Pt-Ni nanocrystals enclosed by stepped surface for methanol oxidation. Chem Sci, 2012, 3: 1925

Sun Y, Xia Y. Alloying and dealloying processes involved in the preparation of metal nanoshells through a galvanic replacement reaction. Nano Lett, 2003, 3: 1569-1572

506 Wittstock A, Zielasek V, Biener J, et al. Nanoporous gold catalysts for selective gas-phase oxidative coupling of methanol at low temperature. Science, 2010, 327: 319-322

507 Cui C, Gan L, Heggen M, et al. Compositional segregation in shaped Pt alloy nanoparticles and their structural behaviour during electrocatalysis. Nat Mater, 2013, 12: 765-771

508 Matanović I, Garzon FH, Henson NJ. Theoretical study of electrochemical processes on Pt-Ni alloys. J Phys Chem C, 2011, 115: 10640-10650
509 Shui J, Chen C, Li JCM. Evolution of nanoporous Pt-Fe alloy nanowires by dealloying and their catalytic property for oxygen reduction reaction. Adv Funct Mater, 2011, 21: 3357-3362

510 Wu Y, Wang D, Zhou G, et al. Sophisticated construction of Au islands on Pt-Ni: an ideal trimetallic nanoframe catalyst. J Am Chem Soc, 2014, 136: 11594-11597

511 Blonder GE. Simple model for etching. Phys Rev B, 1986, 33: 6157-6168

512 Ren F, Wang Z, Luo L, et al. Utilization of active Ni to fabricate $\mathrm{Pt}-\mathrm{Ni}$ nanoframe/NiAl layered double hydroxide multifunctional catalyst through in situ precipitation. Chem Eur J, 2015, 21: 13181-13185

Acknowledgments This work was supported by the Fundamental Research Funds for the Central Universities (WK2060190043 and WK2060190053), and the National Natural Science Foundation of China (21521091, 21131004, 21390393, U1463202 and 21522107).

Author contributions The manuscript was written through contributions of all authors. All authors have given approval to the final version of the manuscript.

Conflict of interest The authors declare that they have no conflict of interest. 

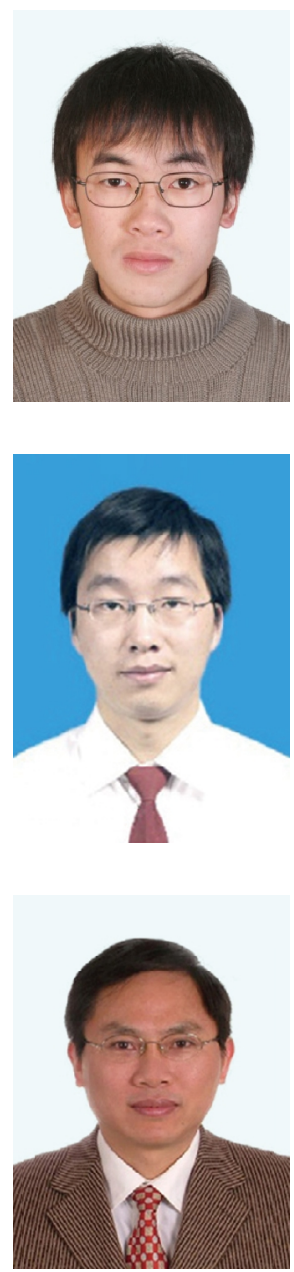

Yuen Wu received his BSc and PhD degrees from the Department of Chemistry, Tsinghua University in 2009 and 2014, respectively. He is currently a professor in the Department of Chemistry, University of Science and Technology of China. His research interests are focused on the synthesis, assembly, characterization and application exploration of functional nanomaterials.
Dingsheng Wang received his BSc degree from the Department of Chemistry and Physics, University of Science and Technology of China in 2004, and his PhD degree from the Department of Chemistry, Tsinghua University in 2009, under the supervision of Prof. Yadong Li. He did his postdoctoral research at the Department of Physics, Tsinghua University, with Prof. Shoushan Fan. He joined the faculty of the Department of Chemistry, Tsinghua University in 2012.

Yadong Li received his BSc degree from the Department of Chemistry, Anhui Normal University in 1986 and his PhD degree from the Department of Chemistry, University of Science and Technology of China in 1998, under the supervision of Prof. Yitai Qian. He joined the faculty of the Department of Chemistry, Tsinghua University in 1999 as a full professor. His research interests are focused on the synthesis, assembly, structure and application exploration of nanomaterials.

\section{理解纳米材料在液相合成中的主要反应}

吴宇恩 ${ }^{1,2}$, 王定胜 ${ }^{1}$, 李亚栋 ${ }^{{ }^{*}}$

摘要 本综述涵盖了涉及纳米材料液相合成的主要反应, 将沉淀法、还原法、晶种生长法、刻蚀法等传统策略分为自下而上和自上而下 两个基本过程. 主要集中讨论了纳米晶成核与生长(尤其在液相体系中)的基本机理和原则. 本文也预测了如何利用这些纳米合成的基本规 律去构造所需的具有特定结构的、功能性的纳米结构. 在合成纳米科学中, 如果研究人员可以熟练掌握主要反应机理、抓住基本技术, 便 可以用更直接、有效的方法去控制纳米晶的结构. 